\title{
Automatic Derivation of LQN Performance Models from UML software models using Epsilon
}

\author{
by
}

Mana Hassanzadeh Zargari

A thesis submitted to the Faculty of Graduate and Postdoctoral Affairs in partial fulfillment of the requirements for the degree of

Master of Applied Science

in

Electrical and Computer Engineering

Carleton University

Ottawa, Ontario

(C) 2016, Mana Hassanzadeh Zargari 


\begin{abstract}
Model-Driven Development (MDD) is an emerging software paradigm that raises the level of abstraction of software development by changing the focus from code to models and automates the generation of code from models. MDD also facilitates the analysis of non-functional properties, such as performance, in the early software development phases. The objective of this thesis is to develop a model transformation process that takes as input a UML software model with MARTE performance annotations, and generates a corresponding Layered Queueing Network (LQN) performance model in a format understood by the existing LQN tools. The transformation is developed in Epsilon, a new family of languages specialized in model transformations, refinement and management.
\end{abstract}




\section{Acknowledgements}

First and foremost, I would like to express my deepest gratitude, sincere appreciation and respect to my thesis supervisor, Dr. Dorina Petriu for her earnest help, devoted support, encouragement and patient guidance through all the stages of this thesis work. It was only through her invaluable supervision that I was able to complete all the work of this thesis. I have been extremely fortunate to have Dr. Dorina Petriu who always made time and effort to respond to all my questions with her patience, constant direction and invaluable advice. I would also like to express my deep and sincere gratitude to my thesis cosupervisor, Dr. Emil Petriu for his continuous help and valuable support throughout this the work of this thesis.

I would like to thank all my dear colleagues and friends from our research group, RADS lab, specially, Ali, Farhana and Azadeh for their help and support. Thank you all. I would also like to thank and appreciate my dear friends Narges, Sali for their friendship and support.

Last but not least, I would like to thank my dear family, my mother Mina, my father Mahmoud and my lovely brother Amirmahdi, for their unconditional love, continuous encouragement and infinite support throughout my life and studies. This thesis is dedicated to them. 


\section{Table of Contents}

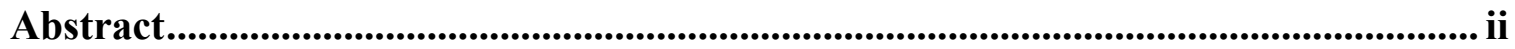

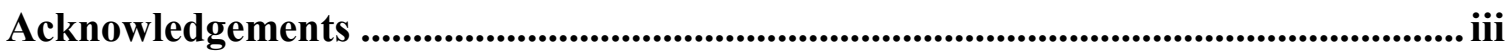

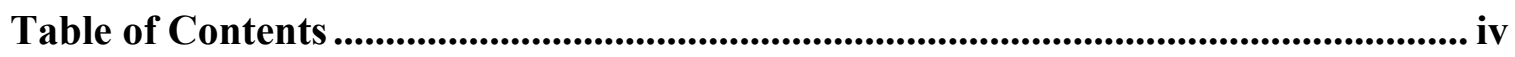

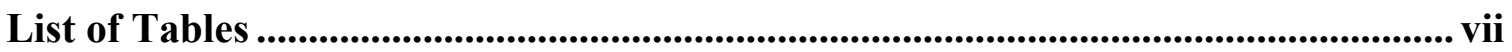

List of Figures.................................................................................................................................... viii

List of Code Fragments ......................................................................................................... $x$

1 Chapter: Introduction ...................................................................................... 1

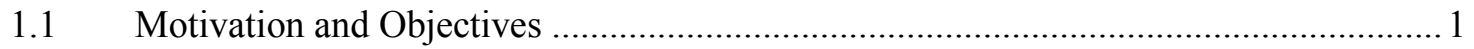

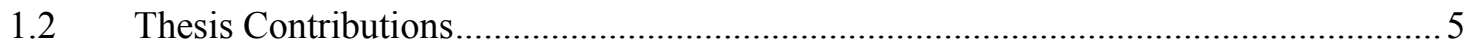

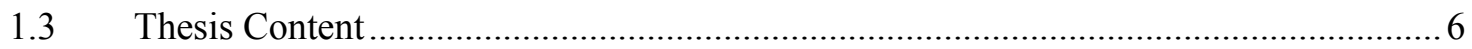

2 Chapter: Background and State of the Art ........................................................ 8

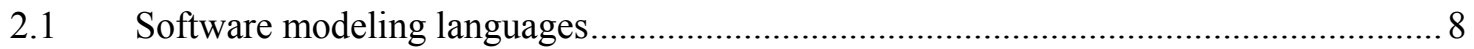

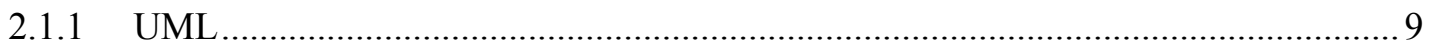

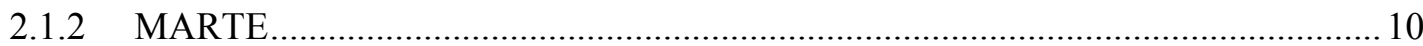

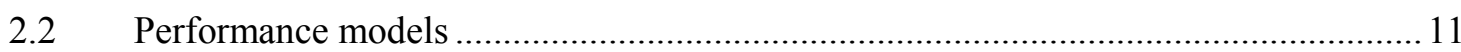

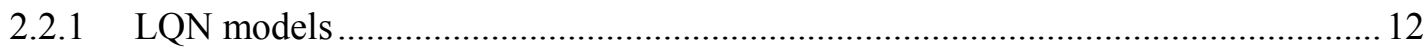

2.3 Deriving performance models from software models ............................................. 13

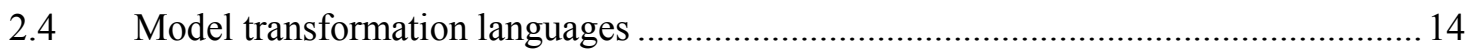

3 Chapter: Source and Target Models.............................................................. 18

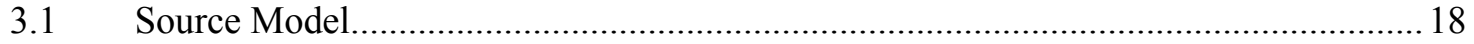

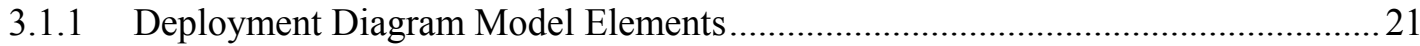

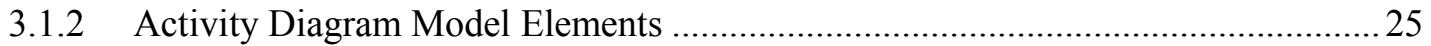

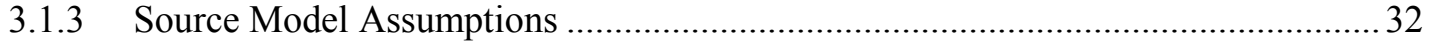

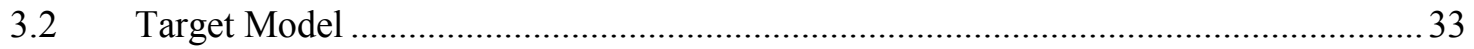


4 Chapter: Transformation Design .............................................................................. 41

4.1 Multi-step process for generating LQN models from UML+MARTE ....................... 41

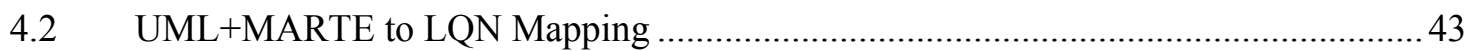

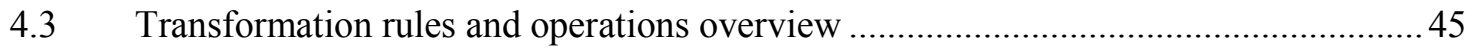

5 Chapter: Transformation Implementation .......................................................... 50

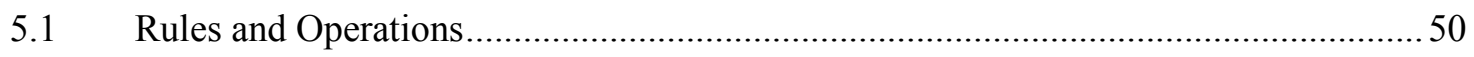

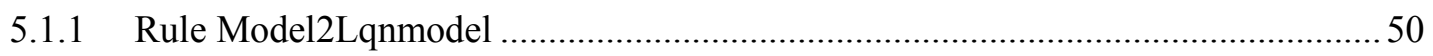

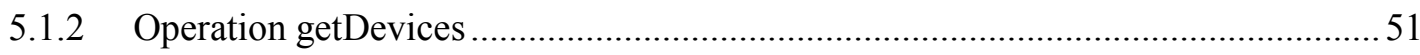

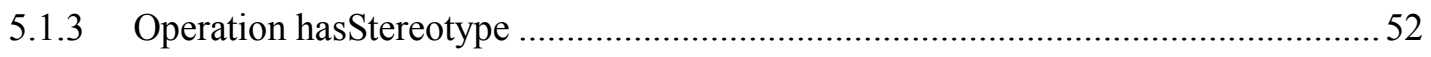

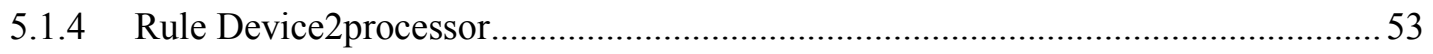

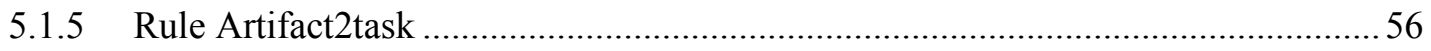

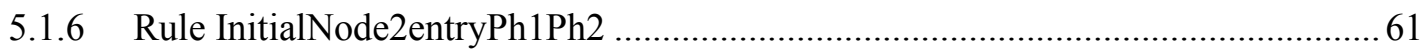

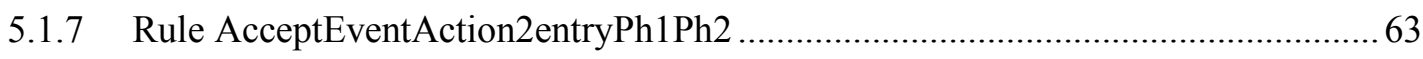

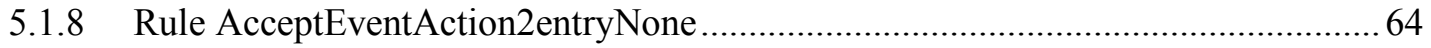

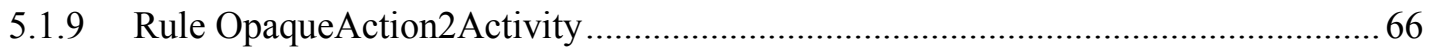

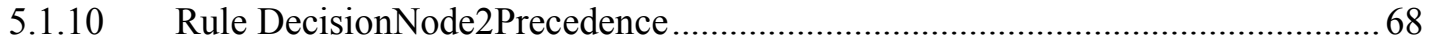

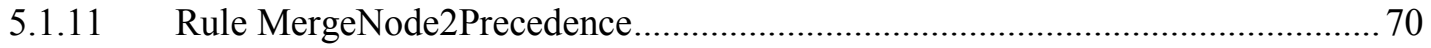

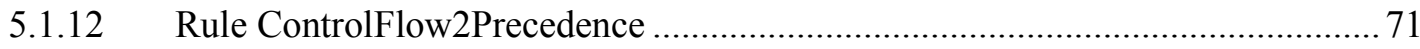

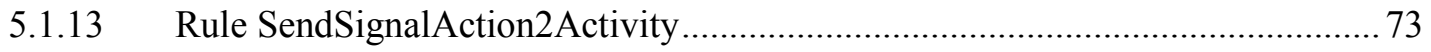

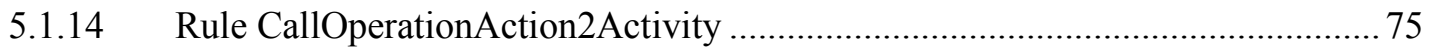

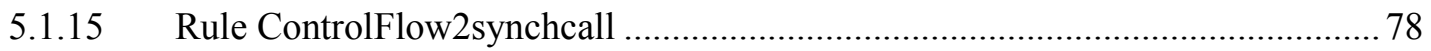

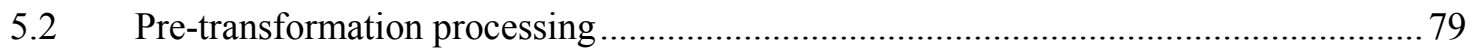

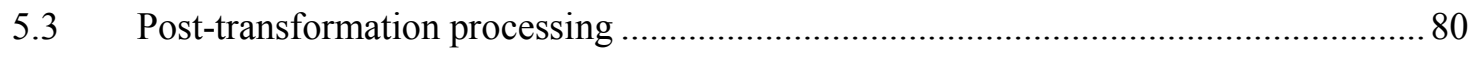

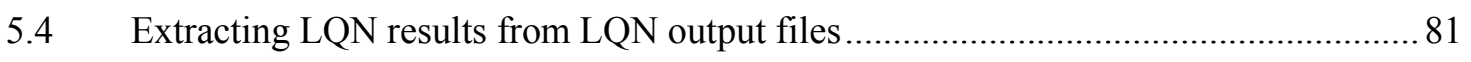

6 Chapter: Transformation Evaluation ..................................................................... 82

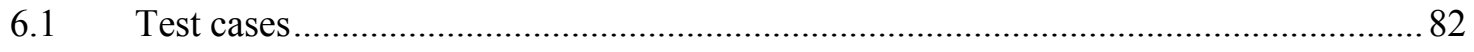


6.2 Case study: E-commerce system......

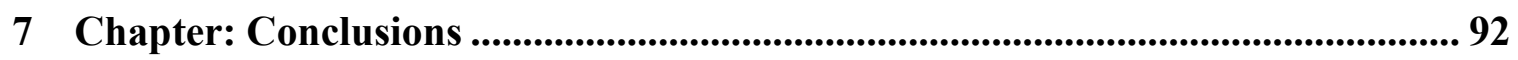

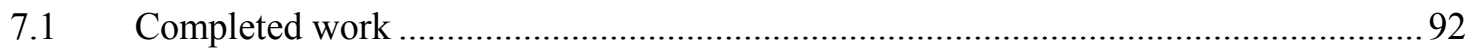

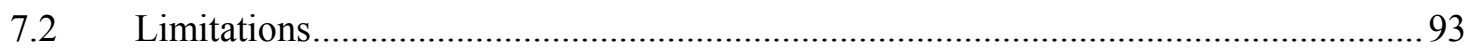

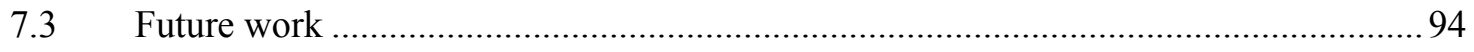

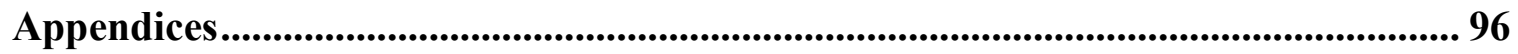

Appendix A LQN Metamodel in Emfatic language ...........................................................96

Appendix B LQN model generated from the UML model from Figures 3-1 and 3-2 2........... 100

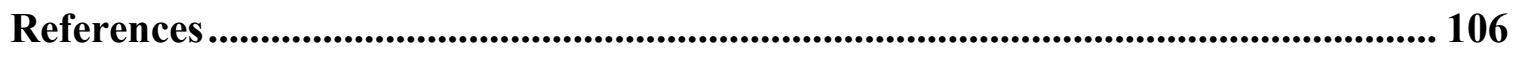




\section{List of Tables}

Table 4-1. Mapping from UML source to LQN target model ...................................... 45

Table 4-2. Transformation Rules for UML to LQN transformation............................. 45

Table 4-3. Operations in UML to LQN transformation............................................... 47

Table 6-1. Test Cases used for verifying different features...................................... 83 


\section{List of Figures}

Figure 3-1. Example of Deployment Diagram of the Source Model................................. 19

Figure 3-2. Example of Activity Diagram of the Source Model ..................................... 19

Figure 3-3. UML2 metamodel: Abstract Syntax of Packages .......................................... 20

Figure 3-4. UML2 metamodel: Abstract Syntax of Nodes ................................................. 21

Figure 3-5. MARTE Generic Quantitative Analysis Modeling (GQAM) Profile for

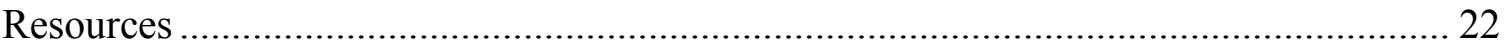

Figure 3-6. UML2 Metamodel: Abstract Syntax of Artifacts........................................... 23

Figure 3-7. MARTE General Resource Modeling (GRM) Profile .................................... 24

Figure 3-8. UML2 Metamodel: Abstract Syntax of ActivityGroups................................. 26

Figure 3-9. UML2 Metamodel: Abstract Syntax of ControlNodes .................................. 27

Figure 3-10. UML Metamodel: Abstract Syntax of Actions .......................................... 28

Figure 3-11. UML2 Metamodel: Abstract Syntax of InvocationActions ........................ 28

Figure 3-12. UML2 Metamodel: Abstract Syntax of AcceptEventActions ..................... 29

Figure 3-13. MARTE/PAM Profile for Workload, Behavior, and Time Observations ... 31

Figure 3-14. UML2 Metamodel: Abstract Syntax of Activities ....................................... 32

Figure 3-15. LQN Schema from [LQN15]. Elements in blue are results found in the output, and elements in red are not implemented in the solver. ...................................... 35

Figure 3-16. LQN metamodel designed for the Epsilon ETL transformation................... 37

Figure 3-17. Graphical notation for the LQN model automatically generated from the

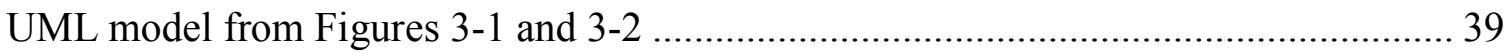

Figure 4-1. Multi-step process for deriving LQN models from UML+MARTE models. 41

Figure 4-2. High-level view of the mapping between the source and target models....... 44 
Figure 5-1. Example of "Device" Element of the Source Model 52

Figure 5-2. Transformation rule example - Device2processor ................................... 54

Figure 5-3. Example of "Artifact" Element of the Source Model ................................. 55

Figure 5-4. Transformation rule example - Artifact2task ...................................... 58

Figure 5-5. Example of "ActivityPartition" Element of the Source Model...................... 58

Figure 5-6. Example of "ControlFlow” Element of the Source Model ........................... 60

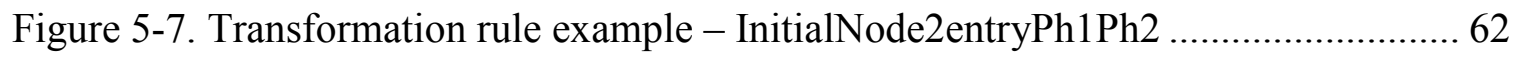

Figure 5-8. Transformation rule example - AcceptEventAction2entryPh1Ph2 .............. 63

Figure 5-9. Transformation rule example - AcceptEventAction2entryNone.................. 65

Figure 5-10. Transformation rule example - OpaqueAction2Activity ........................... 67

Figure 5-11. Transformation rule example - DecisionNode2Precedence ....................... 69

Figure 5-12. Transformation rule example - MergeNode2Precedence.......................... 71

Figure 5-13. Transformation rule example - ControlFlow2Precedence ........................ 73

Figure 5-14. Transformation rule example - SendSignalAction2Activity ..................... 75

Figure 5-15. Transformation rule example - CallOperationAction2Activity ................. 77

Figure 5-16. Transformation rule example - ControlFlow2synchcall.......................... 79

Figure 5-17. Example of Epsilon Console displaying the results ............................... 81

Figure 6-1. Deployment Diagram of E-commerce System ...................................... 88

Figure 6-2. Activity Diagram of BrowseCatalog Scenario ....................................... 89

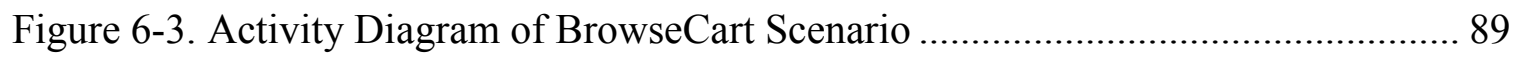

Figure 6-4. Activity Diagram of PlaceOrder Scenario .......................................... 90

Figure 6-5. LQN Model of E-commerce System.................................................. 91 


\section{List of Code Fragments}

Code Fragment 5-1. Transformation rule Model2Lqnmodel .......................................... 50

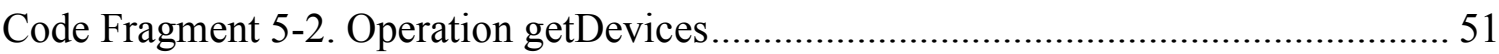

Code Fragment 5-3. Operation hasStereotype ……………............................................. 53

Code Fragment 5-4.Transformation rule - Device2processor.......................................... 53

Code Fragment 5-5. Transformation rule - Artifact2Task ........................................... 56

Code Fragment 5-6. Transformation rule - InitialNode2entryPh1Ph2 …...................... 62

Code Fragment 5-7. Transformation rule - AcceptEventAction2entryPh1Ph2 .............. 64

Code Fragment 5-8. Transformation rule - AcceptEventAction2entryNone................... 64

Code Fragment 5-9. Transformation rule - OpaqueAction2Activity ............................... 66

Code Fragment 5-10. Transformation rule - DecisionNode2Precedence ......................... 68

Code Fragment 5-11. Transformation rule - MergeNode2Precedence ............................ 70

Code Fragment 5-12. Transformation rule - ControlFlow2Precedence........................... 72

Code Fragment 5-13. Transformation rule - SendSignalAction2Activity ........................ 74

Code Fragment 5-14. Transformation rule - CallOperationAction2Activity................... 76

Code Fragment 5-15. Transformation rule - ControlFlow2synchcall............................. 79

Code Fragment 5-16. Example of EOL Script writing to the UML Source Model.......... 80

Code Fragment 5-17. Example of EOL Script reading from LQN Target Model............ 81 


\section{Chapter: Introduction}

\subsection{Motivation and Objectives}

Model-Driven Development (MDD) is an emerging software paradigm that raises the level of abstraction of software development by changing the focus from code to models, and automates the generation of code from models. The thesis research is based on MDD, so we are using new languages, techniques and tools that have been developed to support MDD, such as:

- Modeling languages. Two modeling languages are used in this thesis: a) the Unified Modeling Language (UML) that represents software during the development process; and b) the Layered Queueing Network (LQN) that describes performance models for software systems. UML is standardized by OMG [UML15] and widely used in research and industry, and LQN has been developed at Carleton University [LQN15] and is currently used by many research groups worldwide.

- Metamodels. A metamodel is a special kind of model that defines the abstract syntax of a modeling language. The UML metamodel used in the thesis is the OMG standard implementation (imported as an Eclipse plugin) and the LQN metamodel is defined in the thesis based on the LQN XML schema from [LQN15].

- Model Transformations. A transformation is a special application that takes one or more input models (each defined by a source metamodel), and generates one or more output models (each defined by a target metamodel). A model transformation from UML with performance annotations to LQN is developed in the thesis.

- Specialized model transformation languages. In the past, model transformations were implemented in general purpose languages (such as Java). More recently, a new 
category of languages has been created, specialized for model transformation. A family of such languages called Epsilon [Kol15] is used in the thesis; it is composed of inter-related compatible languages with different purposes (e.g., model transformation, verification and management). It is the first family of model transformation languages and it is expected to have enhanced expressive power.

Beside code generation, the MDD focus on models also facilitates the analysis of nonfunctional properties (NFP), such as performance, scalability, reliability, security, safety, etc. of the software under development based on its model. This brings more “engineering" into software development, leading to the paradigm known as ModelDriven Engineering (MDE).

In the thesis we are interested in verifying a single non-functional property, namely performance. In order to be able to derive a performance model from a UML software model, some extra performance information needs to be added to the model (such as CPU demands for the execution of different parts of the software or number of requests to given services). In the case of UML-based software development, the extensions required for performance annotations can be defined as UML profiles. Two OMG standard UML profiles provide, among other features, the ability to define performance annotations: the UML Profile for Schedulability, Performance and Time (SPT) defined for UML 1.X versions [SPT05] and the UML Profile for Modeling and Analysis of Real-Time and Embedded systems (MARTE) defined for UML2.X versions [MART11].

The work of the thesis is motivated by the following concerns:

- Support performance evaluation in early MDD phases.

- Integrate the automatic derivation of performance models in MDD software process. 
- Insure the seamless inter-operation of multiple tools by using appropriate formats for the information interchange.

- Experiment with a new family of languages, Epsilon, that belongs to a new category of languages specialized in model transformations. We are interested in this new category because of their power of expression. It is expected that these specialized languages are able to bridge a larger semantic gap between the source and target models than regular general purpose languages, creating more compact implementations easier to build and maintain.

Comparison with previous related transformations. Performance from Unified Model Analysis (PUMA) is a performance analysis approach, developed in the Department of Systems and Computer Engineering at Carleton University, which uses an intermediate model for transformations between different kinds of design models to different kinds of performance models [Wood05, Wood14]. The goals of PUMA are to reduce the complexity of the transformations between many design models to many performance models by replacing a large semantic gap with two smaller ones. Initially a UML design model annotated with MARTE is developed by a software engineer, and then this model is transformed into a Core Scenario Model (CSM) intermediate model [Petr07], which may be transformed in turn to different performance models, such as Layered Queuing Networks, Queueing Networks, Petri nets, simulation. Using an existing performance solver, the performance model is analyzed and the performance results are obtained.

PUMA is a rather challenging model transformation that was addressed by two $\mathrm{PhD}$ theses, which developed the two main parts of PUMA: a) the transformation from UML+MARTE to CSM [Alha14]; and b) another transformation from CSM to LQN 
[Petr14]. We have learned from their experience how to approach the mapping between UML and LQN, but have developed the transformation in a different way, at a higher level of abstraction.

Here is a brief comparison of the PUMA approach for generating LQN models from UML+MARTE and the approach described in the thesis:

- The fact that PUMA contains an intermediate model, CSM, brings both advantages (a smaller semantic gap to be bridged by each transformation) and disadvantages (an extra transformation and an extra model).

- The thesis transformation goes directly from UML+MARTE to LQN. The transformation is faster (as there is no need to generate and store an intermediate model) and allows for support of inter-model traceability because cross-model trace links can be defined for each executed rule.

- PUMA was developed in Java, a general purpose language that is not specialized for model transformations. The thesis work is using Epsilon, a new family of languages specialized in model transformations.

- Java programs for model transformations are longer and more complex because more it works at a lower level of abstraction;

- The Java programmer takes care of many details for navigating the source model, recognizing patterns of model elements, verifying conditions and generating target model elements that have to be glued together.

○ Java is imperative, while Epsilon is declarative/imperative hybrid language. The Epsilon engine takes over a number of tasks (such as what rule to apply next) that must be handled explicitly by the Java program. 
○ Epsilon provides high-level support for transformation rules, OCL-like operations, model navigation, and generation of target model elements. This allows the developer to think at a higher level of abstraction and to write shorter programs.

It is expected that the outcome of the thesis work will enable future research with the following objectives:

- To support the import of performance analysis results from the LQNS solver output to the UML+MARTE model.

- To support cross-model traceability between software and analysis models;

- To support the co-evolution of the software and analysis models.

- To support incremental propagation of changes from the source to the target model.

\subsection{Thesis Contributions}

As already mentioned, the main high-level goal of this thesis is to contribute to the seamless integration of software performance engineering in the early stages of the model-driven software development process. More specifically, the first objective of the thesis is to develop a model transformation process that takes as input a UML software model with MARTE performance annotations, and generates a corresponding Layered Queueing Network (LQN) performance model in a format understood by the existing LQN tools [LQN15]. Another objective of the thesis is to experiment with a new family of languages, Epsilon, which is part of a new category of languages, specialized in model transformations, refinement and management. The work for achieving these objectives has resulted in the following contributions: 
- Developing an automatic multi-step LQN model derivation process that includes model transformation, model refining and re-formatting steps. The process starts with a UML software model extended with MARTE performance annotations and generates the corresponding LQN model in XML format. The generated LQN model can be parsed and solved by the existing LQNS solvers, and/or can be transformed in a different existing LQN format (graphical or textual).

- Transformation Design: Mapping UML+MARTE model elements to corresponding LQN model elements and designing a model transformation as a set of Epsilon ETL rules and operations which work together to generate an LQN model. The transformation generates LQN elements directly from UML elements, without using any intermediate model to bridge the semantic gap between source and target.

- Transformation Implementation in Epsilon ETL: implementing the transformation rules and operations in Epsilon ETL and verifying them. The test cases used to verify the correctness of the implementation are also described in the thesis.

\subsection{Thesis Content}

This thesis is composed of seven chapters and two appendices. The description of the content of each chapter is as follows:

Chapter 2, Background and State of the Art, discusses background concepts and some related work in the following topics: Model-Driven Development (MDD); software modeling languages, such as UML and its MARTE profile; performance models, more specifically, LQN models; deriving performance models from software models; and model transformation languages, specifically the Epsilon family of languages used in the thesis. 
Chapter 3, Source and Target Models, presents first the UML source model, its required diagrams and model elements used in the transformation, and the assumptions regarding the construction of the source model. Next, it discusses the LQN target metamodel given in the LQN documentation [LQN15], and then the metamodel used in the transformation, implemented in the thesis using the Emfatic language.

Chapter 4, Transformation Design, describes first the multi-step process for generating LQN models, which contains transformation, refining and re-formatting steps. Next are discussed the transformation principles, leading to the mapping of UML + MARTE models elements to LQN model elements. The transformation rules are based on this mapping; a list of all ETL transformation rules and operations defined for this transformation is given.

Chapter 5, Transformation Implementation, presents the ETL implementation of some mapping rules and operations, describing their role and semantics in details. The refinement and re-formatting steps of the LQN derivation process are also presented.

Chapter 6, Transformation Evaluation, presents some test cases used for of testing the transformation and all the other steps of the process. The transformation is applied to a case study described by other researchers in [Corte11]. The validation of the transformation is performed by showing that the generated LQN models are understood by the existing LQN tools.

Chapter 7, Conclusions, discusses the accomplishments of the thesis, limitations and directions for future work. 


\section{Chapter: Background and State of the Art}

\subsection{Software modeling languages}

Model-Driven Development (MDD) is an emerging paradigm in software development, which moves the primary focus of software development process from code to models, raising the level of abstraction. Important characteristics of MDD are the separation of the software application model from the underlying platform models, and the automatic generation of code from models. Another advantage of using models for software development, besides the automatic code generation, is the fact that it is easier to derive formal analysis models for verifying non-functional properties (NFP), such as performance, reliability, scalability, security, safety, etc. Formal verification of NFP adds more engineering aspects to the software development process, which leads from MDD to Model-Driven Engineering paradigm.

There are some steps that need to be followed for analyzing different NFPs. In the first step, additional information specific to each NFP needs to be added to the software model; in the second step, a model transformation that generates an analysis model from the annotated software model needs to be defined; in the third step, the generated analysis model needs to be analyzed by using existing solvers, and finally in the last step, the feedback will be reported to the designers of the software model. For the analysis of various non-functional properties (NFP), many modeling formalisms and tools have been developed such as queueing networks, stochastic Petri nets, stochastic process algebras, fault trees, formal logic, probabilistic time automata, etc. Thus the research challenge is to bridge the semantic gap between Model-Driven Development, existing formalisms for the analysis of NFPs, and also their tools [Petr15]. 


\subsubsection{UML}

The Unified Modeling language (UML) is a general purpose software modeling language, standardized by the Object Management Group (OMG) [UML15]. UML is defined by a metamodel, which is in turn defined in the MOF language [MOF11] and supports a set of graphical notations which are used by human users during the analysis, design, implementation, code generation, testing and maintenance phases of software systems. UML is used for different activities in the software system development, such specification, visualization, construction, simulation, code generation, verification, testing, and documentation.

UML provides thirteen different diagram types which represent different perspectives of the system under development. A UML diagram is created by a UML modeling tool. In the thesis, we use the UML tool Papyrus [Papy15]. The UML diagrams are classified as structure diagrams and behavior diagrams. A structure diagram captures the structure of a system. For example, fixed system components and their respective properties may easily be modeled using a UML class diagram. The structure diagram type includes: Class diagram, Object diagram, Package diagram, Component diagram, Deployment diagram, and Composite structure diagram. In the thesis, we use Deployment diagrams, which are described in more detail in the next chapter.

Behavior diagrams capture the behavior of a system, subsystem or component. Modeling different states and state transitions of system components or specifying the interaction between the user and the system are only two of many modeling possibilities. The behavior diagram type includes for example the UseCase diagram, StateChart, Activity diagram, Sequence diagram, Timing diagram, Communication diagram, and 
Interaction overview diagram. In the thesis we use Activity diagrams to represent the scenarios that are analyzed (described in more detail in the next chapter).

\subsubsection{MARTE}

Although UML supports a rich metamodel which meets the requirements of a wide range of software systems, the modeler may require creation of new modeling elements, or defining additional properties which may not be defined in the UML metamodel. The UML shortage can be solved by either changing the UML metamodel by adding new metaclasses, or by defining an additional profile. Although adding new metaclasses offers a high degree of flexibility, it leads to losing the existing tool support, as standard tools do not recognize the new metaclasses. On the other hand, defining a new profile seems to be the best choice since it does not change the UML metamodel, which means the existing UML tools still can be used.

UML supports three types of built-in extension mechanisms: 1) stereotypes used to extend different UML metaclasses, 2) tagged values which define the properties (or attributes) of the stereotypes, and 3) constraints used to refine the semantics of a UML model element. These three extension mechanisms help the modelers to extend the UML modeling capabilities by adding new concepts, new properties, and new semantics which tailor UML to a specific problem domain. UML also has different standard profiles which specialize UML for different application domains; SPT and MARTE are such examples.

The UML Performance Profile for Modeling and Analysis of Real-Time and Embedded Systems (MARTE) [MART11] extends UML with timing concepts, resources and workload required for quantitative performance and schedulability analysis. The MARTE 
stereotypes and properties used in the thesis are explained in more detail in the next chapter.

\subsection{Performance models}

A performance models is an abstract representation of a real system that captures its performance properties, mostly related to the quantitative use of resources during runtime, and is capable of determining its performance. The model can be used to study the performance impact of different design and/or configuration alternatives under different workloads, leading to advice for improving the system. Performance evaluation of a model may be done either by solving a set of equations by some analytical (possibly numerical) methods or by simulating the model and collecting statistical results. Analytical performance models are usually based on underlying stochastic models, which are often assumed to be Markov processes. A Markov process is a stochastic process with discrete state space, where all information about the future evolution of the process is contained in the present state, and not on the path followed to reach this state. Markov models suffer from a problem known as state space explosion, whereby its number of states grows combinatorially with the performance model size. This may introduce severe limitations in the size of performance models that can be solved.

Examples of well-known analytical performance models are queueing networks, stochastic Petri nets, stochastic automata networks and stochastic process algebra.

Queueing Network (QN). One of the best known performance models captures very well the contention for resources (Lazowska et al., 1984). Efficient analytical solutions exist for a class of QN (known as separable or product-form QN). This make it possible to derive steady-state performance measures without building the underlying state space. 
The advantage is that the solution is faster and larger models can be solved. The disadvantage consists stricter model assumptions (e.g., service time distributions, arrival process, scheduling policies). Similar to the approach for product-form QN, approximate solutions have been developed for non-separable QN. There are many extensions to QN in literature. One of them is the Layered Queueing Networks, which is the target model of the model transformation in the thesis.

Stochastic Petri Nets (SPN) (Ajmone Marsan et al., 1995) are very good flow models able to represent concurrency, but not as good at representing resource contention and especially queueing policies. Efficient solutions exist only for a limited class of SPN; most interesting models are solved with Markov chain-based solutions.

Stochastic Automata Networks (Plateau et al., 1991) are composed of modular communicating automata synchronized by shared events and executing actions with random execution times. The main disadvantage is the state space explosion of its Markovian solution.

Stochastic Process Algebra, introduced in (Hillston, 1994), takes a compositional approach by decomposing the system into smaller subsystems easier to model. This approach is based on enhanced process algebra, Performance Evaluation Process Algebra (PEPA). The compositional nature of the language provides benefits for model solution as well as model construction. The solution is based on the underlying Markov process.

\subsubsection{LQN models}

The target model of this transformation is the Layered Queueing Network (LQN) performance model [Fran99][Fran09], that has been developed as a combination of Stochastic Rendezvous Networks [Wood95] and the Method of Layers [Roli95]. The 
LQN model and its solvers (analytic and by simulation) [Fran95][Fran99] have been developed and extended at Carleton University over the past 25 years, and now are used by many research groups around the world. According to a recent survey paper [Danc15] presented at ICPE'2015 presenting a systematic review of the papers published in the proceedings of the International Conference on Performance Engineering (ICPE) and its predecessors, LQN was the only performance modeling language referred to in every single volume of the ICPE+WOSP conference proceedings (14 volumes so far). (ICPE is the premier conference on software performance engineering, established by the joining of the ACM Workshop on Software and Performance (WOSP) and the SPEC International Performance Evaluation Workshop (SIPEW)). The LQN metamodel and its features are described in the next chapter.

\subsection{Deriving performance models from software models}

Software Performance Engineering (SPE), first presented in [Smit90], is a systematic, quantitative approach to construct software systems that meet performance objectives. It is based on the careful and methodical assessment of performance attributes throughout the lifecycle, from requirements and specification to implementation and maintenance [Smit01]. In the software performance engineering field there have been significant efforts to integrate performance analysis into the software development process by using different performance modeling formalisms: queueing networks, Petri nets, stochastic process algebras, and simulation. A good survey of the techniques for deriving performance models from UML models is given in [Bals04] and later in the book [Cort11]. Here are a few early examples of derivation of different kinds of performance models from UML. The technique presented in [Cort00] follows the SPE 
methodology very closely, generating the same kind of models as in [Smi90]. In [Lope04] UML models are transformed into Petri Nets, but the contention for hardware resources is not considered. [Cave04] presents a transformation from UML to Stochastic Process Algebra.

The performance research group from Carleton University has implemented UML-toLQN transformations in different languages and was the first to use the standard UML metamodel libraries which were current at the time and the standard performance profiles SPT [SPT2005] and MARTE [MART11]. A solution described in [Amer01] uses a graph-rewriting tool called PROGRES, based on graph-grammars. Another solution presented in [Petr02] implements in Java an ad-hoc graph transformation at the UML 1.4 metamodel level. A two-phase transformation from UML to LQN using XML algebra concepts and implemented in XSLT was presented in [Gu05].

The most comprehensive model transformation of the Carleton group, which took as input a number of different software models (including UML+SPT and UML+MARTE) generated a number of target performance models (such as LQN, QN and Petri nets) is the PUMA transformation [Wood05, Wood14] and its extensions for Service-oriented Architecture, PUMA4SOA [Alha10, Alha14].

\subsection{Model transformation languages}

Most approaches to model transformations have been based on general purpose programming languages, (mostly Java, which is used in the open-source Eclipse Modeling Framework). However, more recently specialized model transformation languages have also been developed. In 2002, the Object Management Group (OMG) issued a Request for Proposal (RFP) on MOF Query/View/Transformations to develop a 
standard transformation language compatible with other OMG standards (UML, MOF, OCL, etc.) Several replies to the RFP were submitted by a number of companies and research institutions; the replies have evolved into the QVT standard language [QVT08].

QVT is the OMG standard model transformation language that mainly focuses on model query, view and transformation. It supports two levels of declarative language (the Relations and the Core) and an imperative language (the Operational mapping). The Relations language is used in matching object patterns, tracing model elements and creating object templates. The Core language is considerably simpler than the Relations language and is used in matching patterns where a set of variables are evaluated against a set of models [OMG08]. The Operational mappings language is used for invoking the imperative representations of transformations from the Relations or the Core.

ATL. One of the model transformation languages proposed during the OMG standardization process was the Atlas Transformation Language (ATL). ATL was released earlier than MOF QVT and has acquired a considerable group of users who helped apply ATL and evolve its toolset [ATL12].

Epsilon (standing for Extensible Platform of Integrated languages for mOdel maNagement) is a platform aimed to create a family of consistent and interoperable taskspecific languages, able to handle model management tasks such as model transformation, code generation, model comparison, merging, refactoring and validation [Kol15]. Epsilon framework contains Epsilon Object Language (EOL), Epsilon Validation Language (EVL), Epsilon Transformation Language (ETL), Epsilon Comparison Language (ECL), Epsilon Merging Language (EML), Epsilon Wizard Language (EWL), and Epsilon Generation Language (EGL). Epsilon also provides a set 
of ANT tasks to support creation of workflows of different tasks. Epsilon programs interacts with models which conforms to concrete modeling technologies such as EMF and XML, because Epsilon Model Connectivity layer (EMC) supports these technologies and provides abstraction facilities for them [Kol15].

In this thesis, we decided to use the Epsilon family of languages. More specifically, we used Epsilon Object Language (EOL), which provides a reusable set of common model management facilities, and Epsilon Transformation Language (ETL). All the task specific languages are extensions of EOL and are supported by EOL. Also, EOL can be used as a standalone language for the purpose of model management [Kol15].

ETL adds the capability of model-to-model transformation to the Epsilon framework. At a higher level of abstraction, ETL transforms an arbitrary number of input models into an arbitrary number of output models of different modeling languages and technologies [Kol15].

Model transformation languages are classified in three different categories or styles: declarative, imperative and hybrid. Declarative transformation languages provide better support for cases in which the source and target metamodels are similar to each other in terms of structure, so that the transformation is a simple mapping between two metamodels, but are not as powerful when complex mapping is required. Imperative transformation languages are capable of supporting more complex transformation cases, however, they operate at a lower level of abstraction, meaning that issues such as tracing and resolving target elements, and orchestrating the transformation execution are addressed by the users. In order to address the shortcomings of declarative and purely imperative languages mentioned above, hybrid languages such as ATL and QVT are 
defined, each providing a declarative rule-based execution scheme as well as imperative features to handle complex transformation [Kol15]. ETL also is a hybrid language which implements a task-specific rule definition and execution scheme. It also inherits the imperative features of EOL for handling complex transformations [Kol15]. 


\section{Chapter: Source and Target Models}

\subsection{Source Model}

The source model of the transformation developed in the thesis represents a software system expressed in UML 2.5 [UML14] extended with the MARTE profile and especially with its Performance Analysis Modeling (PAM) subprofile. In this section the UML diagrams composing the model and their main elements used in the transformation rules are described, along with the stereotypes applied to them. This section also explains in details how the UML model elements and MARTE stereotypes are used in the source model, showing how the source model is required to be created in order to be compatible with the transformation. A list of assumptions summarizes the way in which a source model is expected to be constructed. The UML diagrams composing a source model are a Deployment diagram, which represents the system structure and one or more Activity diagrams annotated with $M A R T E / P A M$ performance annotations, which represent the system behavior. Figure 3-1 and Figure 3-2 show examples of deployment and activity diagrams respectively, which together form a source model accepted by transformation developed in the thesis. A UML Deployment diagram represents the runtime architecture of a system and shows how the software components are allocated to hardware processing nodes [UML15]. A UML Activity is a kind of Behavior, which is specified as sequencing of subordinate units in the form of a graph composed of ActivityNodes interconnected by ActivityEdges [UML15]. In our case, a UML Activity Diagram is used to model the flow of execution steps that compose a scenario; a model may contain one or more scenarios, each represented by an activity diagram. 

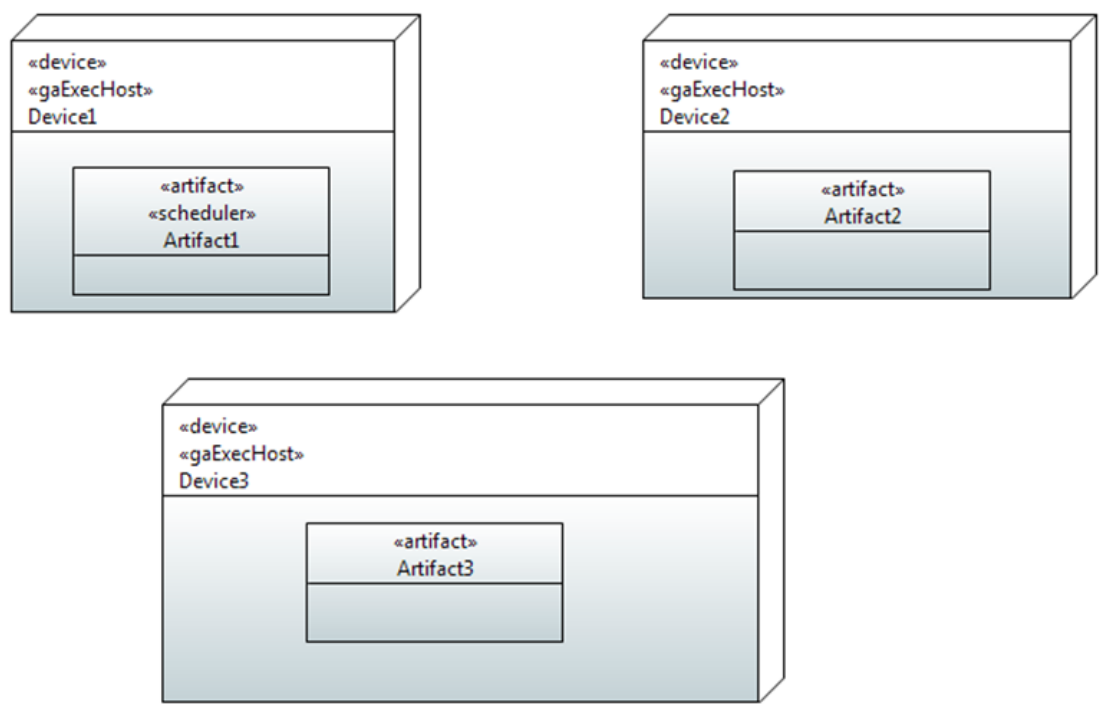

Figure 3-1. Example of Deployment Diagram of the Source Model

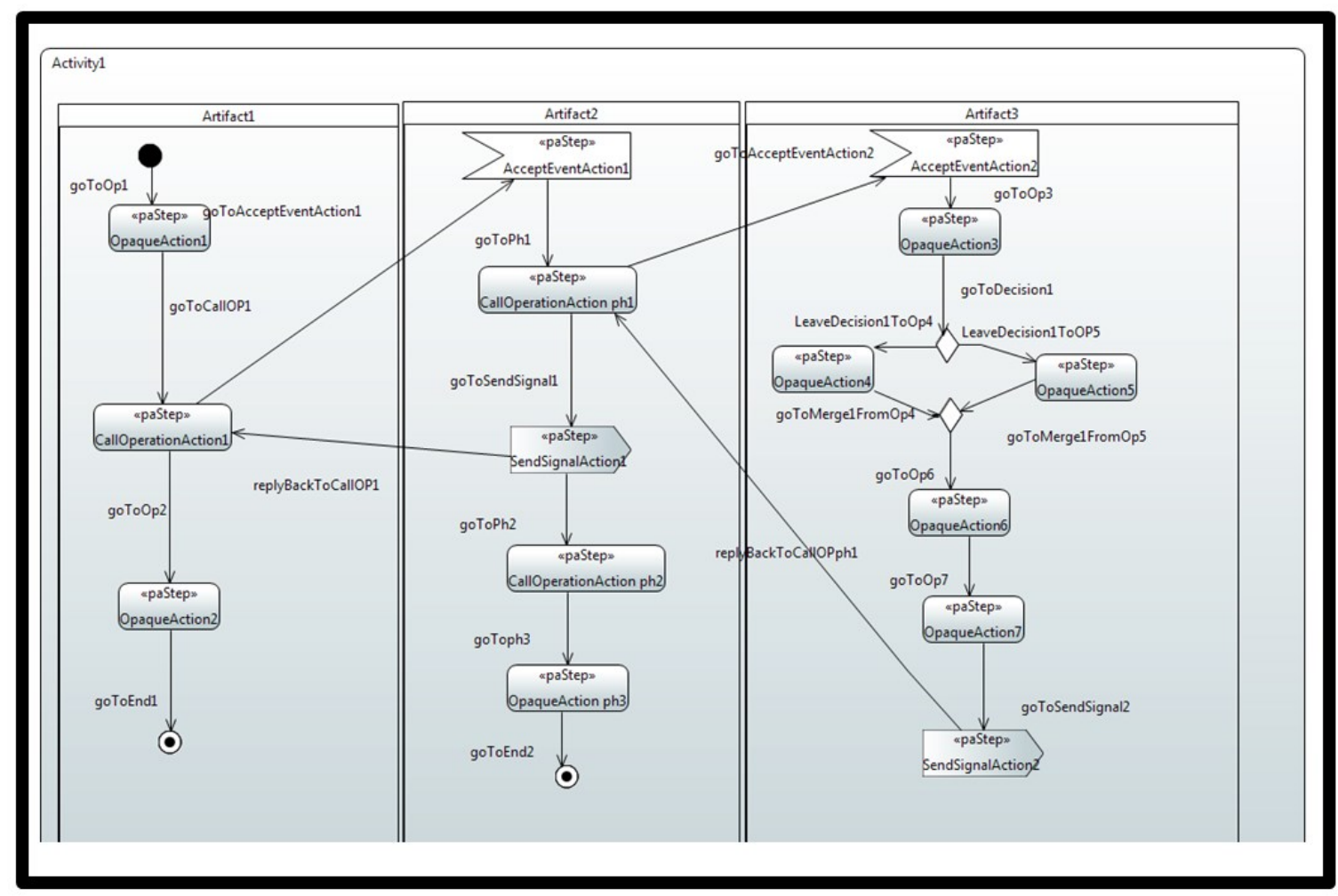

Figure 3-2. Example of Activity Diagram of the Source Model 
The main UML elements of the deployment and activity diagrams used in the transformation together with the UML metamodel fragments defining them, as well as the corresponding applied MARTE stereotypes, are briefly described in the following. All the UML metamodel diagrams and MARTE Profile diagrams presented in this section were taken from the OMG UML standard document [UML15] and OMG UML Profile for MARTE standard document [MART11] respectively.

Root Model Element. According to the UML standard definition, Model is the root element of a model representing a system, where system can be not only software and hardware, but also organizations and processes. A model represents a view of the system, and describes the system from a certain perspective (viewpoint) at a certain level of abstraction with a certain purpose, which decides what is relevant to be included in or

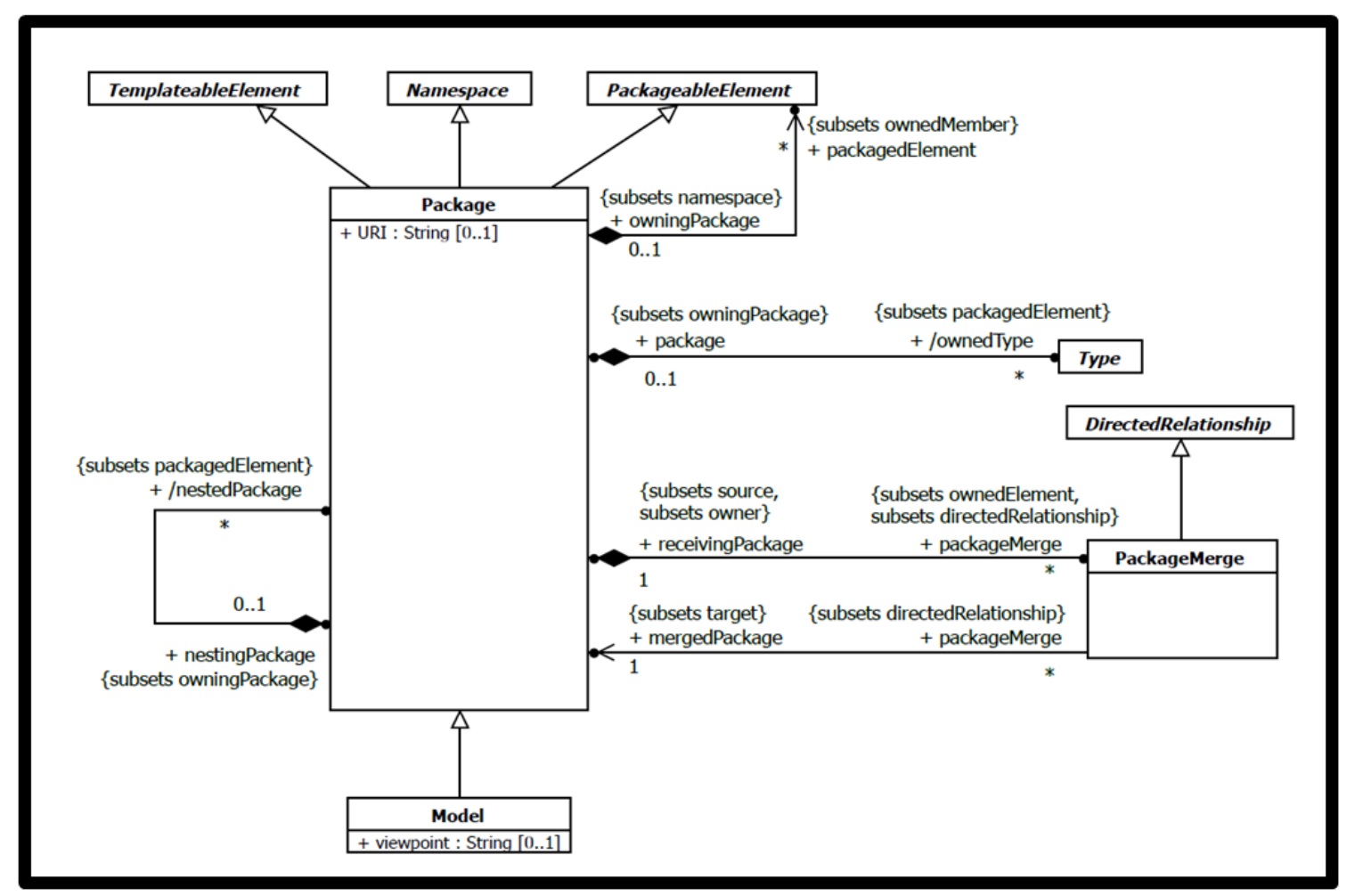

Figure 3-3. UML2 metamodel: Abstract Syntax of Packages 
is irrelevant and needs to be excluded from the Model. According to the definition in the UML metamodel [UML15], Model is a subclass of UML Package as in Figure 3-3 that shows a snapshot of the UML2 metamodel for UML Packages. As a Package, it has a set of members that together describe the modeled system. In our case, the modeled system should contain a structural description that shows how the runtime software components are allocated to hardware devices, and behavior description for one or more scenarios selected for performance analysis.

\subsubsection{Deployment Diagram Model Elements}

Device: A Device is a special kind of UML Node, which is a physical computational resource with processing capability. Artifacts (representing software components) may be deployed on Devices for execution [UML15]. Figure 3-4 shows a snapshot of the abstract syntax of UML Nodes. UML Device has a property namespace, which has to be set to the UML Model containing it for the purpose of the transformation.

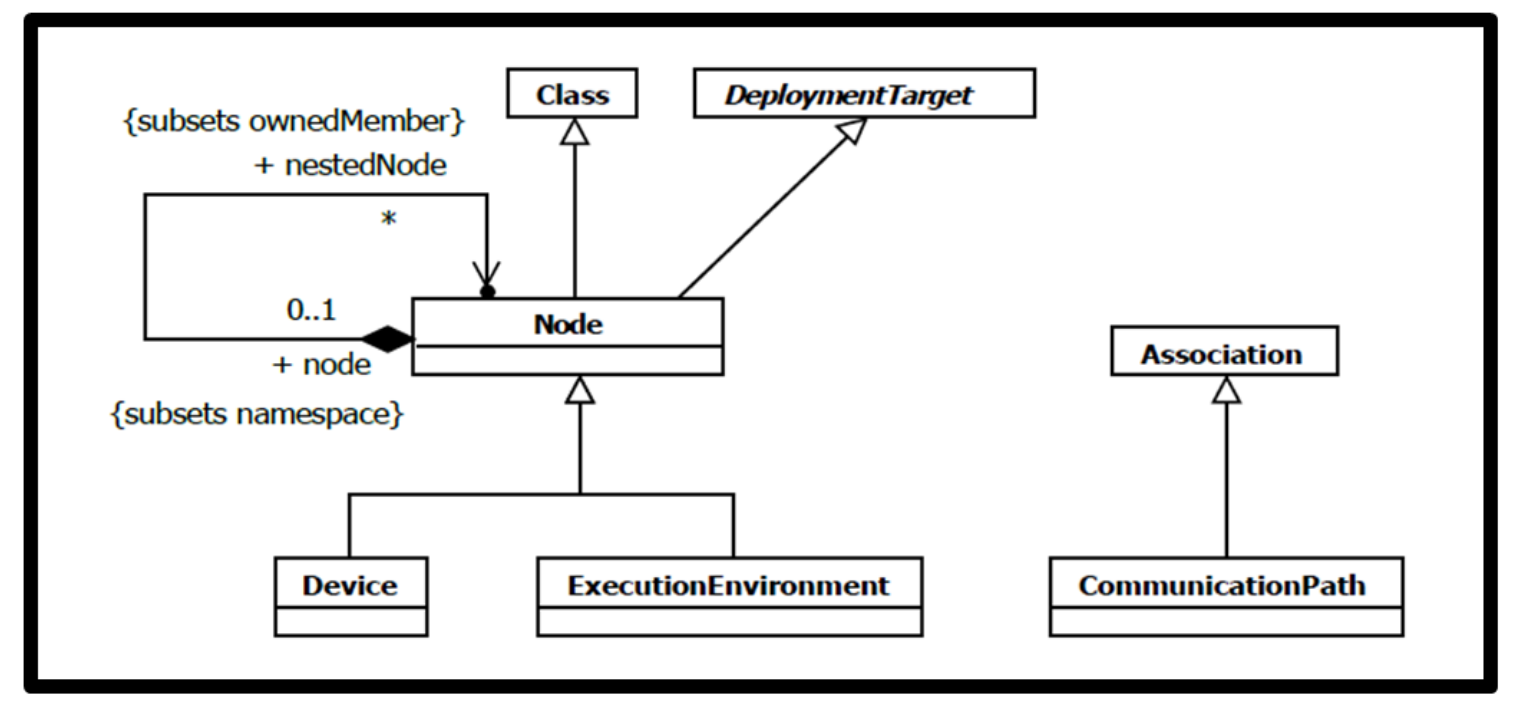

Figure 3-4. UML2 metamodel: Abstract Syntax of Nodes

GaExecHost Stereotype: This MARTE stereotype maps the elements of the ExecutionHost domain to UML Nodes. GaExecHost specifies the characteristics of the 
processing resource that executes scenario steps. It represents either a physical processor or the operating system which support an application at a more abstract level [Seli13] [MART11]. In our case, the GaExecHost is used in the deployment diagram (see Figure 3-1). Figure 3-5 shows the diagram of the MARTE Generic Quantitative Analysis Modeling (GQAM) Profile containing the GaExecHost stereotype. This diagram shows UML extensions for $G Q A M$ stereotypes related to resources.

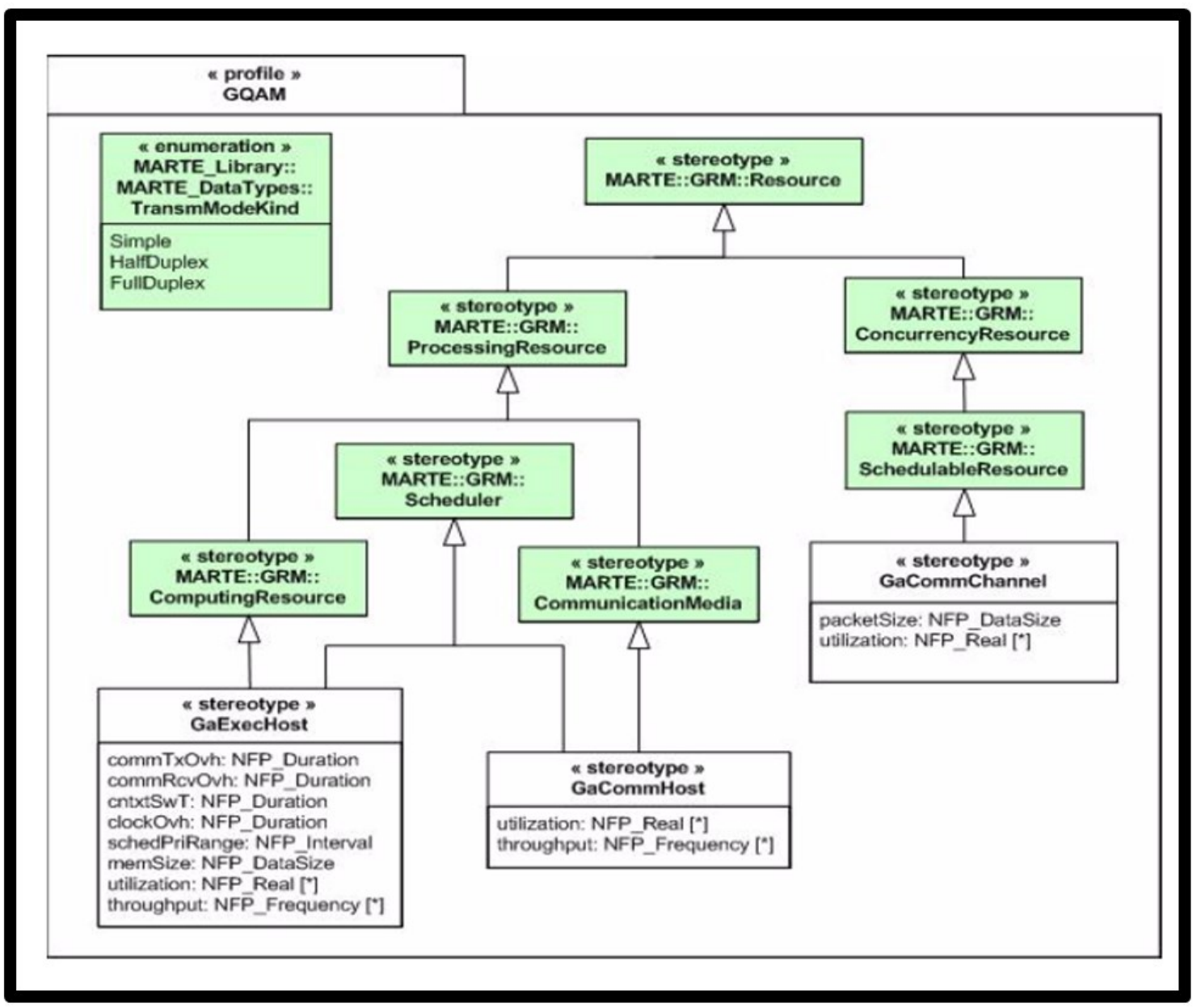

Figure 3-5. MARTE Generic Quantitative Analysis Modeling (GQAM) Profile for Resources

Artifact: an Artifact specifies a software item, which is produced or used by a software development process or a system deployment. It is an elaboration of the UML abstract notion of DeployedArtifact by representing concrete elements in the physical 
world. Model files, source files, binary executable files, scripts, a table in a database system, a development deliverable, a word-processing document, and a mail message are examples of Artifacts [UML15]. In our case, artifacts are used to represent software components allocated to hardware devices (see Figure 3-1). Figure 3-6 depicts the abstract syntax of Artifacts. The property namespace of UML Artifact has to be set to the UML Device which is containing it for the purpose of the transformation.

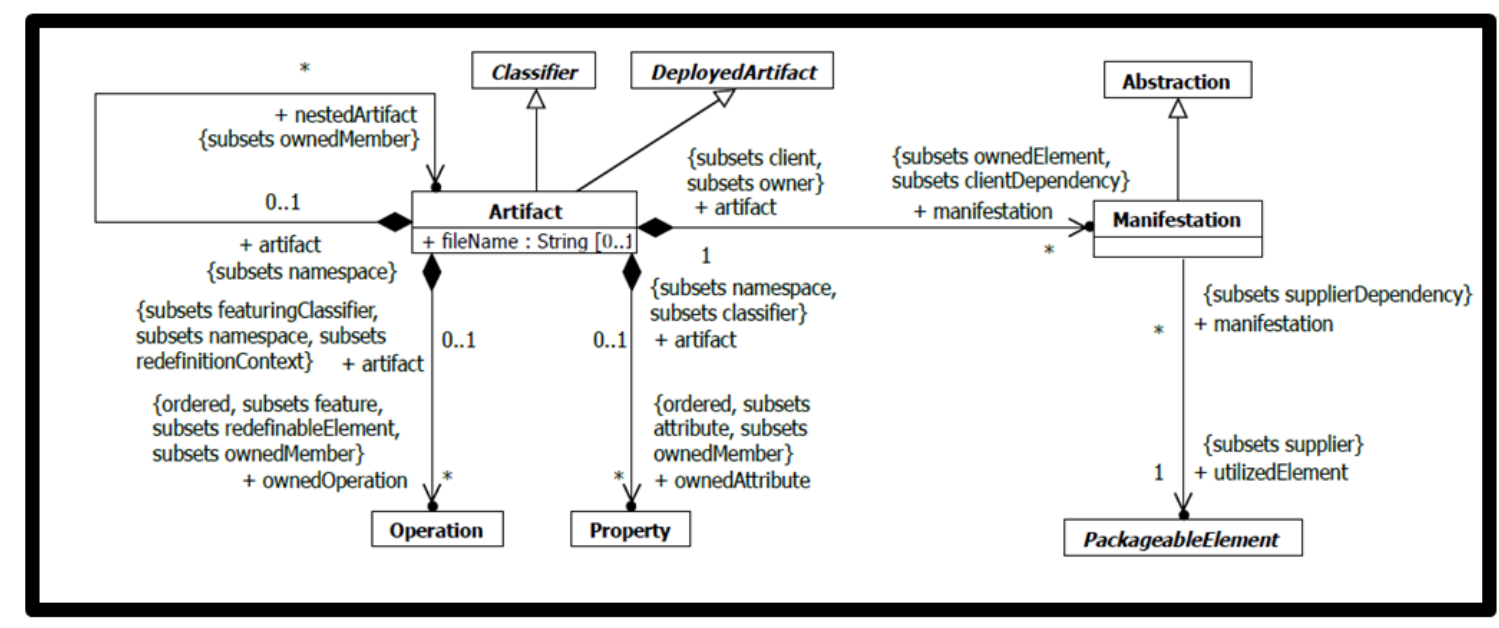

Figure 3-6. UML2 Metamodel: Abstract Syntax of Artifacts

Scheduler Stereotype: A Scheduler Stereotype is a kind of ResourceBroker which brings access to its brokered ProcessingResource or resources which have a certain scheduling policy [MART11]. For the purpose of our transformation, Scheduler stereotype is applied directly to the UML Artifact element to be able to use its otherSchedPolicy (which is of type String and should be initialized to a String value). We decided to use this stereotype to be able to give any scheduling policy value used in the LQN domain to the otherSchedPolicy property. The otherSchedPolicy has to be initialized to the "ref" value for at most one of the Artifact elements from the UML model. The "ref" value indicates a ReferenceTask in LQN, which represents customers in 
the LQN model that drive the system by making requests to other tasks; however, they cannot receive any request [LQN15]. At least one Artifact with "ref" scheduling policy has to be specified in the source UML model in order to generate a LQN model that can be solved by the LQN Solver.

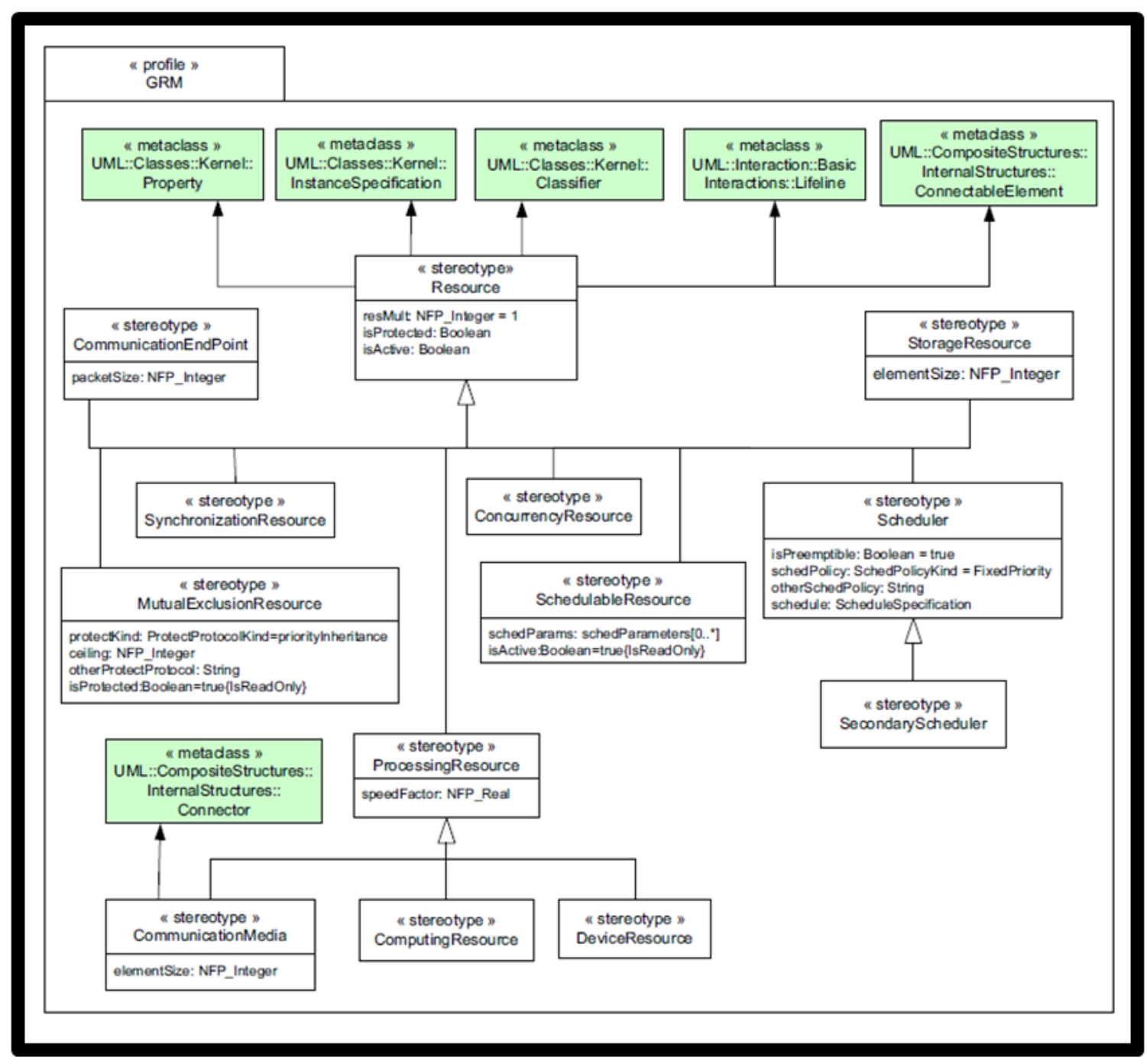

Figure 3-7. MARTE General Resource Modeling (GRM) Profile

An Artifact could be stereotyped by SwSchedulableResource stereotype, which represents an instance of a software task that can be scheduled by an operating system [MarteBook,Bran]. Both SwSchedulableResource and Scheduler stereotypes are kinds of Resource stereotype. It would have been better if we could use SwSchedulableResource 
to stereotype the Artifact element in order to indicate the "ref" scheduling policy. Unfortunately, SwSchedulableResource does not have a scheduling property that we could access directly, so we took the design decision to use the Scheduler stereotype instead. Figure 3-7 shows a diagram of MARTE General Resource Modeling (GRM) Profile, containing the Scheduler stereotype.

\subsubsection{Activity Diagram Model Elements}

Activity Partition: An ActivityPartition is a kind of UML ActivityGroup to identify ActivityNodes which have some characteristics in common. It is noted with two parallel lines and its name attribute is labeled in a box at its top. This notation for an ActivityPartition is called swimlane. For the purpose of our transformation, for each Artifact defined in Deployment Diagram, there should be a corresponding ActivityPartition in each Activity Diagram to which the artifact participates. Please note that an Artifact may contribute to more than one Activity Diagram, which means that it may have more than one corresponding ActivityPartitions. The correspondence between an Artifact and its corresponding ActivityPartition is made by their respective name attributes, which have to be initialized by the modeler to identical values. Figure 3-8 shows a snapshot of an abstract syntax of ActivityGroups. 


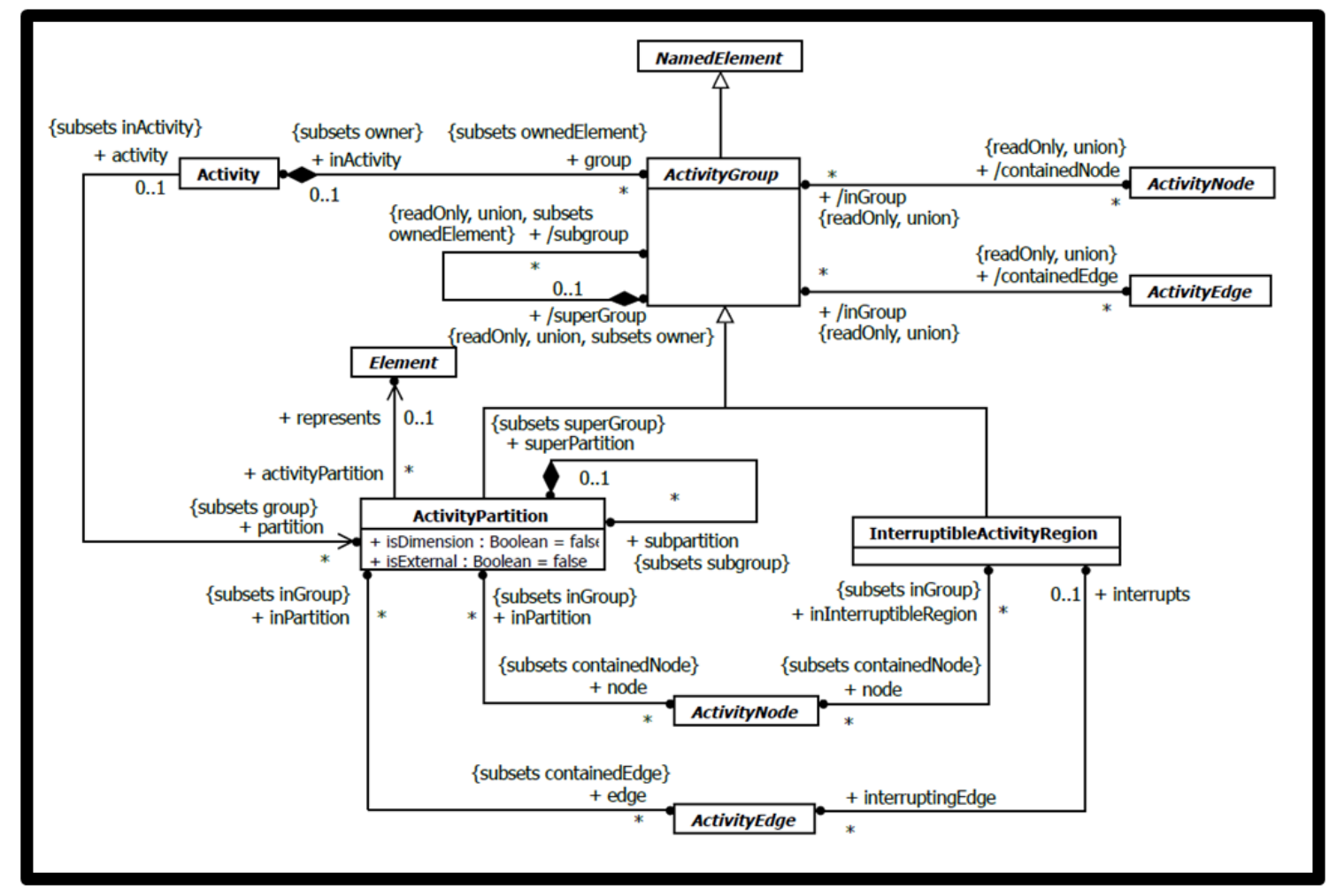

Figure 3-8. UML2 Metamodel: Abstract Syntax of ActivityGroups

Initial Node: an InitialNode is a kind of ControlNode which can be defined as a point to start the execution of an Activity. Execution flow can also start at other nodes, so InitialNodes are not necessarily needed as a starting point. As soon as the Activity starts its execution, the InitialNode becomes enabled, so it does not have any incoming ActivityEdge to be activated [UML15]. Figure 3-9 shows the abstract syntax of ControlNodes.

Activity Final Node: An ActivityFinalNode is a kind of UML FinalNode that terminates all flows in an Activity. A FinalNode is an abstract UML ControlNode at which a flow stops in an Activity. A FinalNode does not have any outgoing ActivityEdges. If there are more than one ActivityFinalNode in an Activity, the first one 
which is reached terminates all flows in the activity [UML15]. Figure 3-9 shows a snapshot of the abstract syntax of ControlNodes.

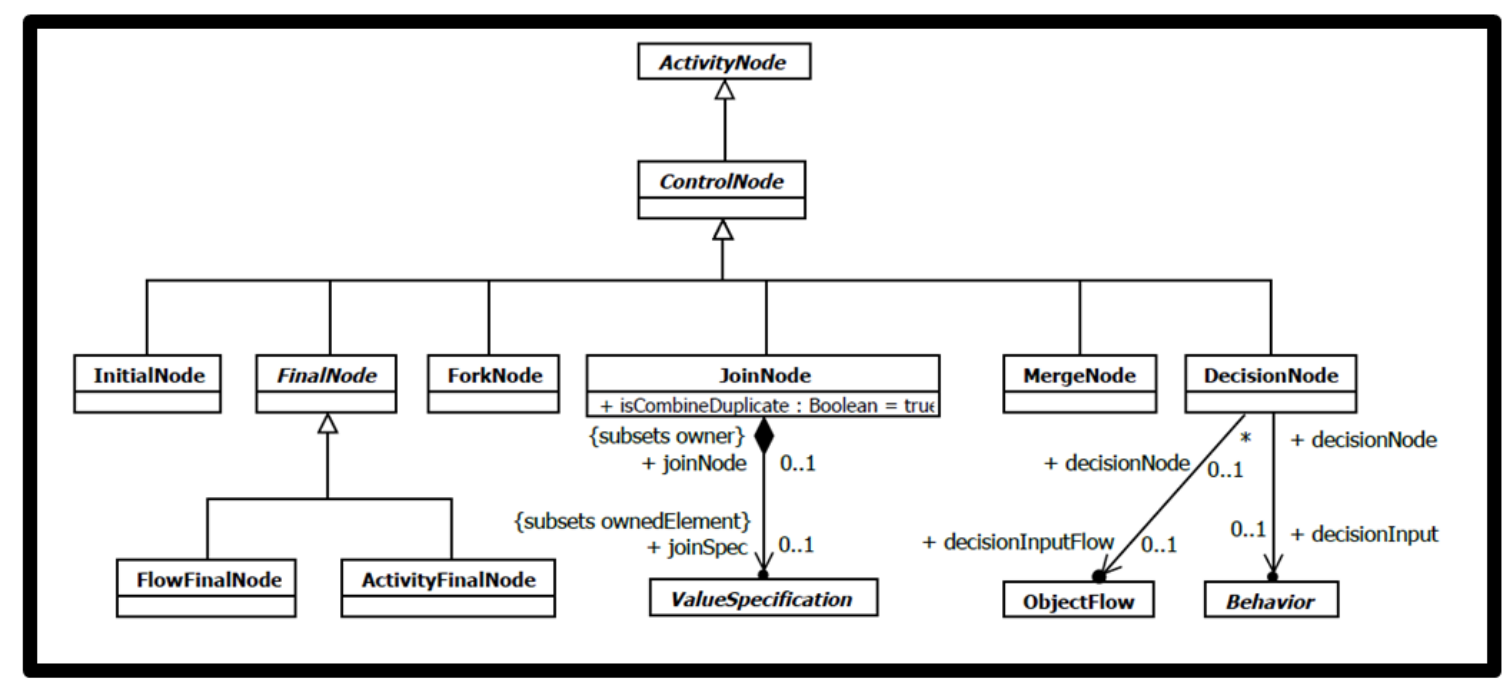

Figure 3-9. UML2 Metamodel: Abstract Syntax of ControlNodes

Opaque Action: An OpaqueAction is a kind of UML abstract Action class. Its specification can be given in a textual concrete syntax other than UML and it can be used as a temporary placeholder before choosing any other kind of Action. An Action which is a kind of UML abstract ExecutableNode, is a fundamental unit of execution within a Behavior [UML15]. Figure 3-10depicts the abstract syntax of Actions.

Call Operation Action: CallOperationAction is a kind of UML CallAction which transmits a message of an Operation Call request to the target object. A CallAction is an InvocationAction which calls a Behavior or an Operation. An InvocationAction is a kind of Action, which results in the invocation of a Behavior directly or indirectly [UML15]. Figure 3-11 shows the abstract syntax of InvocationActions. 


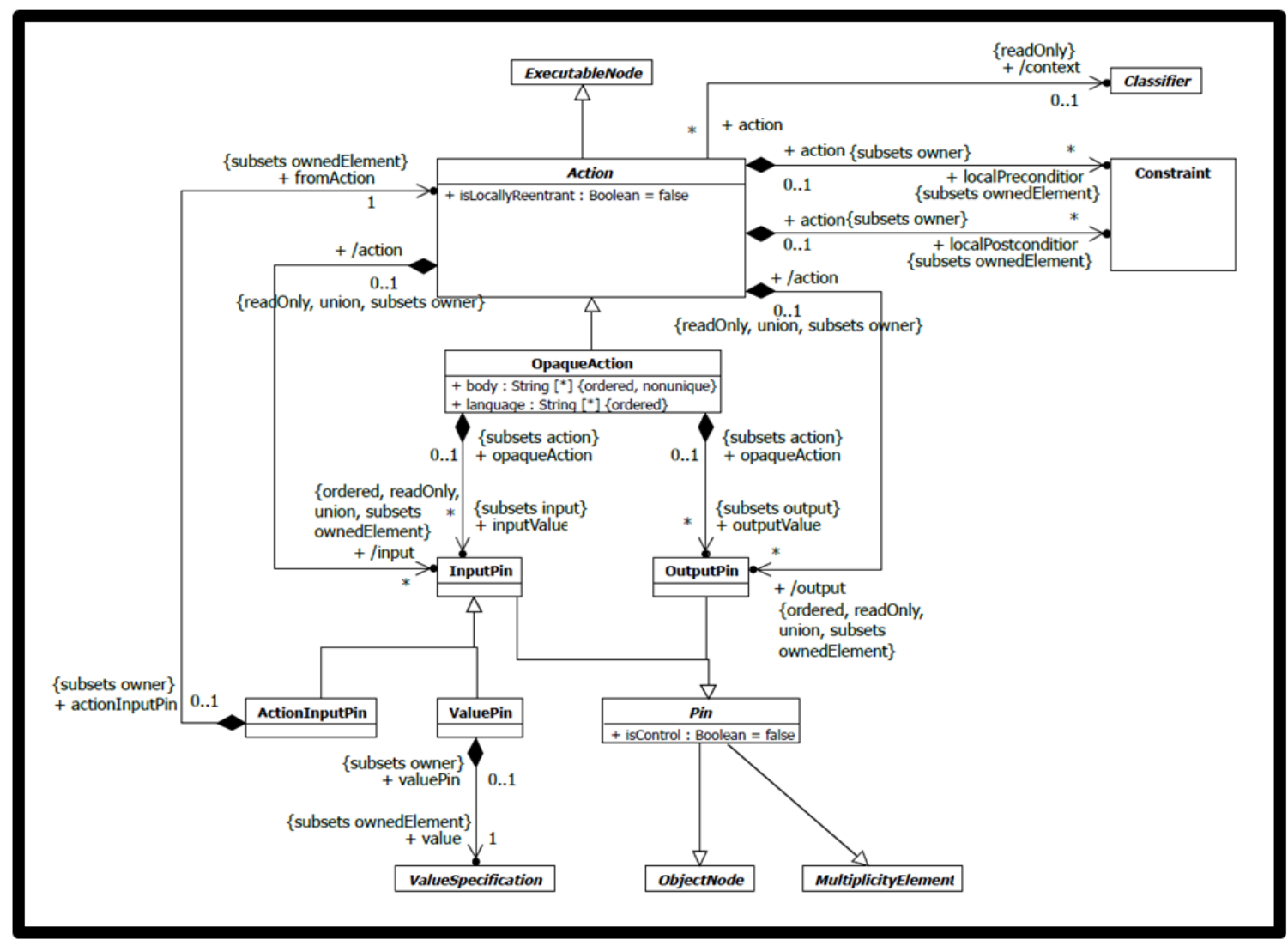

Figure 3-10. UML Metamodel: Abstract Syntax of Actions

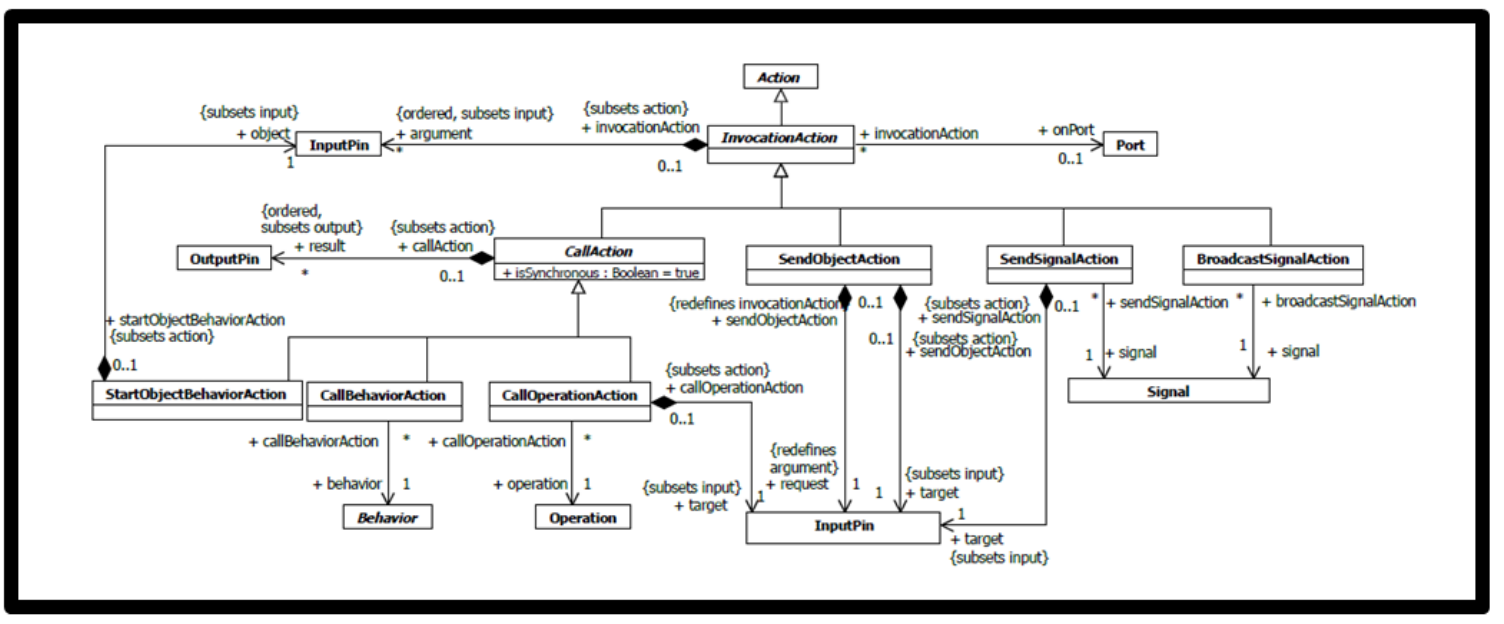

Figure 3-11. UML2 Metamodel: Abstract Syntax of InvocationActions

Accept Event Action: An AcceptEventAction is an Action with triggers for one or more Events. It waits for an Event occurrence to be dispatched from the event pool. When the matching Event occurrence is dispatched from the event pool, the AcceptEventAction 
becomes enabled to continue. If an Event occurrence is for a CallEvent, then a ReplyAction may be used to reply to it. Activities can execute other Actions while AcceptEventActions are waiting (that is why it is called a waiting point). An AcceptEventAction has triggers for CallEvents but it cannot accept synchronous calls unless it is an AcceptCallAction. An AcceptCallAction is a special kind of AcceptEventAction for the handling of CallEvent occurrences [UML15]. However for the purpose of this transformation, we used AcceptEventAction to trigger and handle CallEvents because of the incomplete implementation of the Papyrus tool that does not support AcceptCallAction elements yet. Figure 3-12 shows the abstract syntax of AcceptEventsActions.

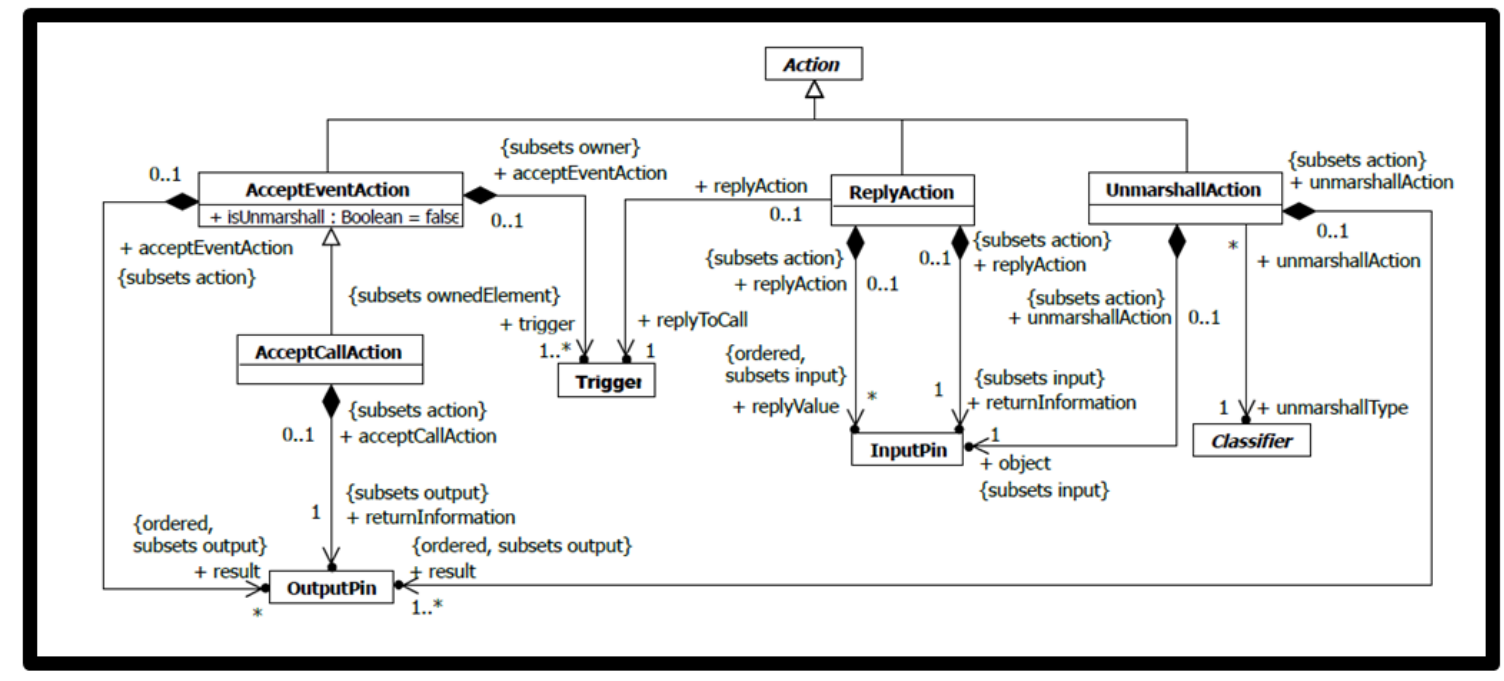

Figure 3-12. UML2 Metamodel: Abstract Syntax of AcceptEventActions

Send Signal Action: A SendSignalAction is a kind of an InvocationAction, which creates a signal instance from its input and transmits the instance to the target object. It is also one of the kinds of send Actions. A send Action is an Action that transmits an object asynchronously. The Action completes as soon as the object is sent to the target. For the 
purpose of this transformation a SendSignalAction is used to complete the handling of an Event call that was accepted by a previous AcceptEventAction. If we could use an AcceptCallAction to receive the call request, A ReplyAction could be used instead of the SendSignalAction. Since A ReplyAction is an Action which handles the completion of the accepted call by an AcceptCallAction [UML15]. However, as it was mentioned before, the current implementation of Papyrus tool does not support the creation of AcceptCallAction and ReplyAction. Figure 3-11 depicts the abstract syntax of InvocationActions.

PaStep Stereotype: The MARTE PaStep stereotype is a special kind of GaStep stereotype. Typically, it is applied to a UML Action or a Message invoking a behavior. A step in a scenario represents a unit of behavior which needs some resources; hostDemand is one of its attributes, which represents a CPU demand [MARTE/Book]. Figure 3-13 shows a the MARTE Performance Analysis Modeling (PAM) Profile, which defines the PaStep stereotype. This diagram shows the PAM performance extensions related to workload, behavior, and time observations.

Fork Node, Join Node, Decision Node, and Merge Node: They are all kinds of UML ControlNode. A ForkNode splits a flow into multiple concurrent flows. A JoinNode synchronizes multiple flows. A DecisionNode chooses between outgoing flows. A MergeNode brings together multiple alternative flows without synchronization. There is no synchronization of flows or joining tokens, so that it accepts one of the alternative incoming flows [UMLDoc/2015]. Figure 3-9 shows a snapshot of an abstract syntax of ControlNodes. 


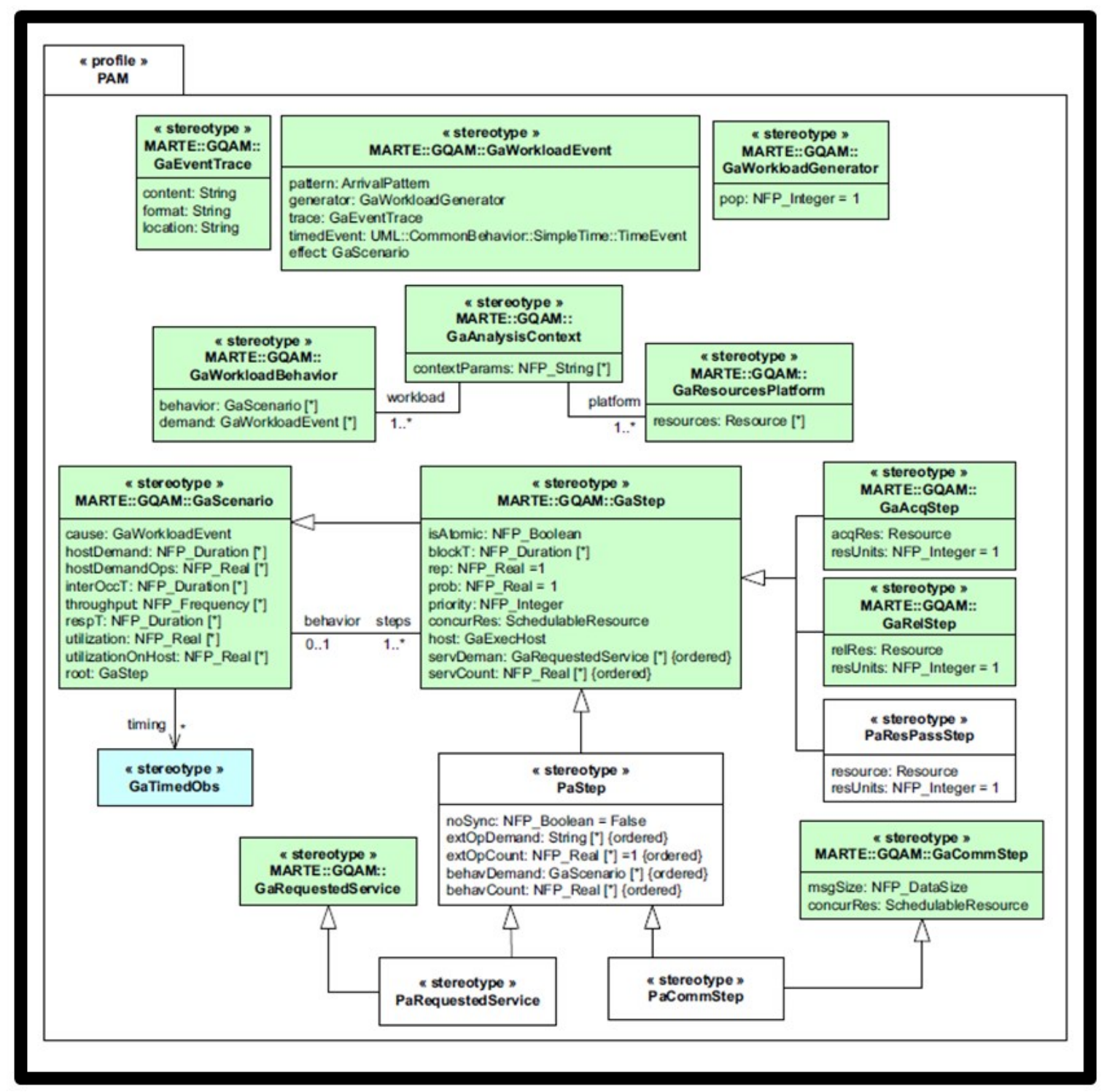

Figure 3-13. MARTE/PAM Profile for Workload, Behavior, and Time Observations

Control Flow: a ControlFlow is an ActivityEdge that only passes control tokens.

They are used to show execution of ActivityNodes in sequence, so that the target ActivityNode will not get a control token and begin its execution until the source ActivityNode completes its execution and creates the token. An ActivityEdge is an abstract class to specify a directed connection between two ActivityNodes. It has to important properties: source (showing the ActivityNode it originates from) and target 
(showing the destination ActivityNode). Tokens which may flow along the ActivityEdge are offered to it by the source ActivityNode [UML15]. A ControlFlow has the property inPartition, which is required to be set for the purpose of the transformation. This property is set for each ControlFlow which is not border-crossing meaning that it is located inside the ActivityPartition and does not cross the swimlane's border. Figure 3-14 shows the abstract syntax of Activities, which contains the ControlFlow metaclass.

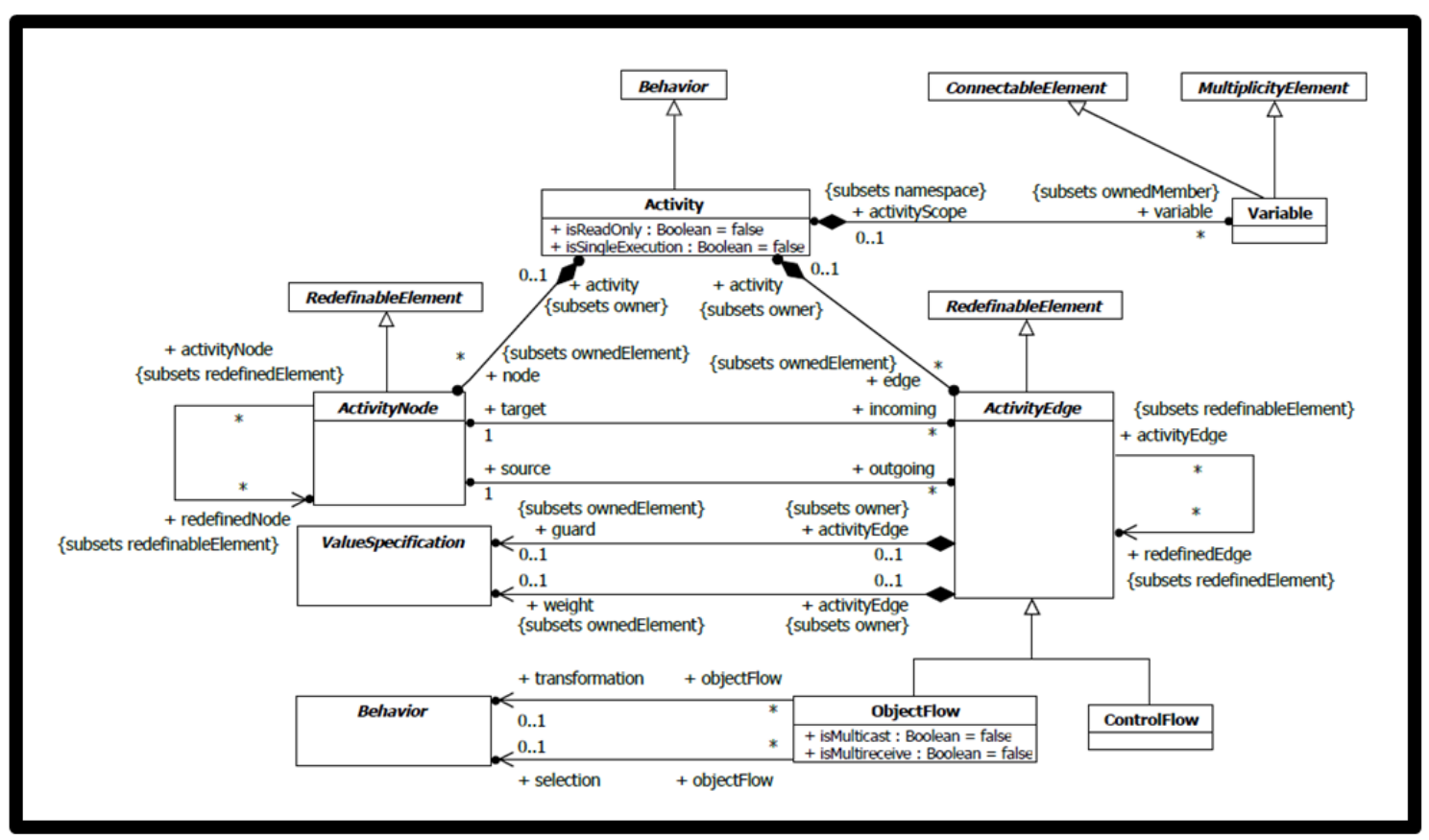

Figure 3-14. UML2 Metamodel: Abstract Syntax of Activities

\subsubsection{Source Model Assumptions}

In order to obtain the expected transformation results using this transformation, the source model needs to be complete and it is required to satisfy the following assumptions:

- Device assumptions: For each Device element, its namespace property needs to be initialized to the UML Model element containing it. Each Device needs to be 
annotated by GaExecHost stereotype and its otherSchedPolicy property could be given a string value.

- Artifact assumptions: For each Artifact element, its namespace property has to be initialized to the Device element containing it. An Artifact that will be transformed to a ReferenceTask in the LQN model, has to be stereotyped with Scheduler stereotype, whose otherSchedPolicy property has to be given a string value of "ref".

- ActivityPartition assumptions: Each Artifact from the Deployment Diagram must have defined at least one corresponding ActivityPartition inside an Activity Diagram. Also, the name attribute of each ActivityPartition should be identical to the name attribute of its corresponding Artifact.

- ControlFlow Assumptions: For each ControlFlow defined inside an ActivityPartition, its inPartition property has to be set to its corresponding ActivityPartition. For those ControlFlow representing call requests, which are not inside a single ActivityPartition but cross the border between ActivityPartitions, the inPartition property does not have to be set.

- CallOperationAction, AcceptEventAction, SendSignalAction, and OpaqueAction Assumptions: Each has to be annotated with PaStep stereotype, and their hostDemand, rep and prop properties should be initialized accordingly.

\subsection{Target Model}

The target model of this transformation is the Layered Queueing Network (LQN), a well known performance model [Fran99][Fran09]. LQN has a graphical notation very useful to the human analysts (see for example Figure 3-17) and two textual formats understood by the existing solvers: a) an older textual version defined by an extended 
BNF grammar; and b) a newer XML version defined by an XML schema. The starting point for LQN analysis is to build manually or automatically an LQN input file in one of the two textual formats. The graphical representation can be produced automatically by using a set of commands that transform an LQN input file into graphical output, such as PostScript or Windows Enhanced Meta File [LQN15].

Since the objective of the thesis is to develop a model transformation able to automatically generate LQN models in a format understood by the existing LQN solvers, we decided to aim for generating LQN models in XML format, because XML is preferred by the model-driven techniques for information interchange between tools.

Figure 3-15, taken from [LQN15], shows the XML metamodel for Layered Queueing Networks using a UML class diagram, which reflects the XML schema for LQN and its containment hierarchy. In XML the layered models are defined in a bottomup manner. The hierarchical structure of an LQN model starts from the lqn-model root block, inside of which are defined the processor blocks. Then all the tasks which are running on a processor are contained within the respective processor block. Each task block contains all the entries which are associated with the task. Similarly, within a task block activity graphs specified by task-activities blocks can be defined. Hence an entry block and task-activities block are siblings in the hierarchy. An entry block may contain entry-phase-activities blocks. Within each entry-phase-activities block, phases are defined (note that the type of a phase is an activity). It should be noted that an entry may contain an activity graph specified by entry-activity-graph tags; however this element is not supported yet by any of the existing LQN solvers. 


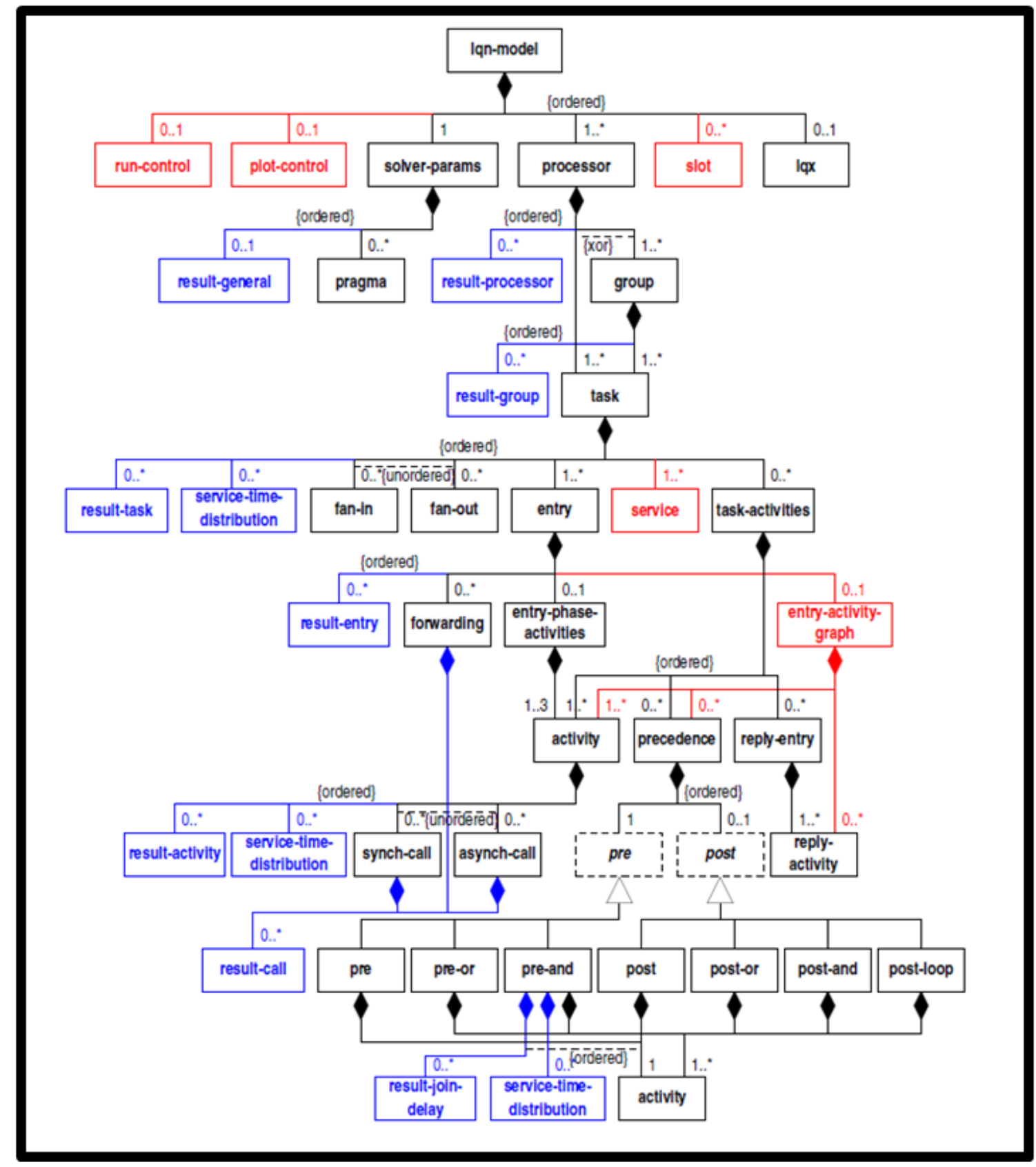

Figure 3-15. LQN Schema from [LQN15]. Elements in blue are results found in the output, and elements in red are not implemented in the solver.

In this XML schema, the elements are ordered from left to right meaning that, for example, a solver-params block comes before a processor block in the XML input file. A 
multiplicity of zero for an association connecting to a model element makes it to be an optional element. Optional elements shown in blue are results of the XML output file and are part of the schema; however, such elements are never present in the LQN input files our transformation generates. Elements in red are not implemented yet and not supported by the solvers, so we ignored them. In order for an XML file to be valid, it must conform to the schema. According to [LQN15], the XML schema enforces the following validity constraints: all processors, tasks and entries have a unique name, respectively. Also within a specific task, all activities have a unique name. There is only one activity which is bound to each entry. All types of calls, synchronous, asynchronous and forwarding must have a valid destination. All activity connections defined in a precedence block should refer to valid activities. All activity replies must refer to a valid entry. All activity loops must refer to valid activities. A multiplicity for phases is from 1 to 3 . Within an entry, all attributes of a phase must be unique [LQN15].

An Epsilon transformation requires access to the source and target metamodels expressed in EMF Ecore (the metamodeling language of the underlying platform Eclipse EMF [EMF15]). Hence, we had to define the LQN metamodel in EMF Ecore. The Eclipse framework offers a language called Emfatic, designed to represent EMF Ecore models in textual form. The first step was to design the LQN metamodel shown in Figure 3-16, then to express it in Emfatic. The new metamodel is derived from the XML-based LQN metamamodel given in the LQN user manual [LQN15] (see Figure 3-15). There are some differences: the new metamodel does not consider the following elements that appear in the XML-based LQN metamodel: a) the red elements (not supported by the tools); b) the blue elements (needed in the output file produced by the solvers, but not for 
the input files that we generate); c) the forwarding call (left for future work) and d) postloop (also left for future work). A small difference between the two metamodels is that all the association roles are named in Figure 3-16, to allow for a complete definition of the association ends (i.e., of their multiplicity and their attachment to classes by composition or simple association). Other small difference is the fact that the Emfatic identifiers cannot contain the dash character '-'. The last difference is the introduction of a new element activity $R$, used instead of activity to define the children of precedence operators (the reason for this is explained below).

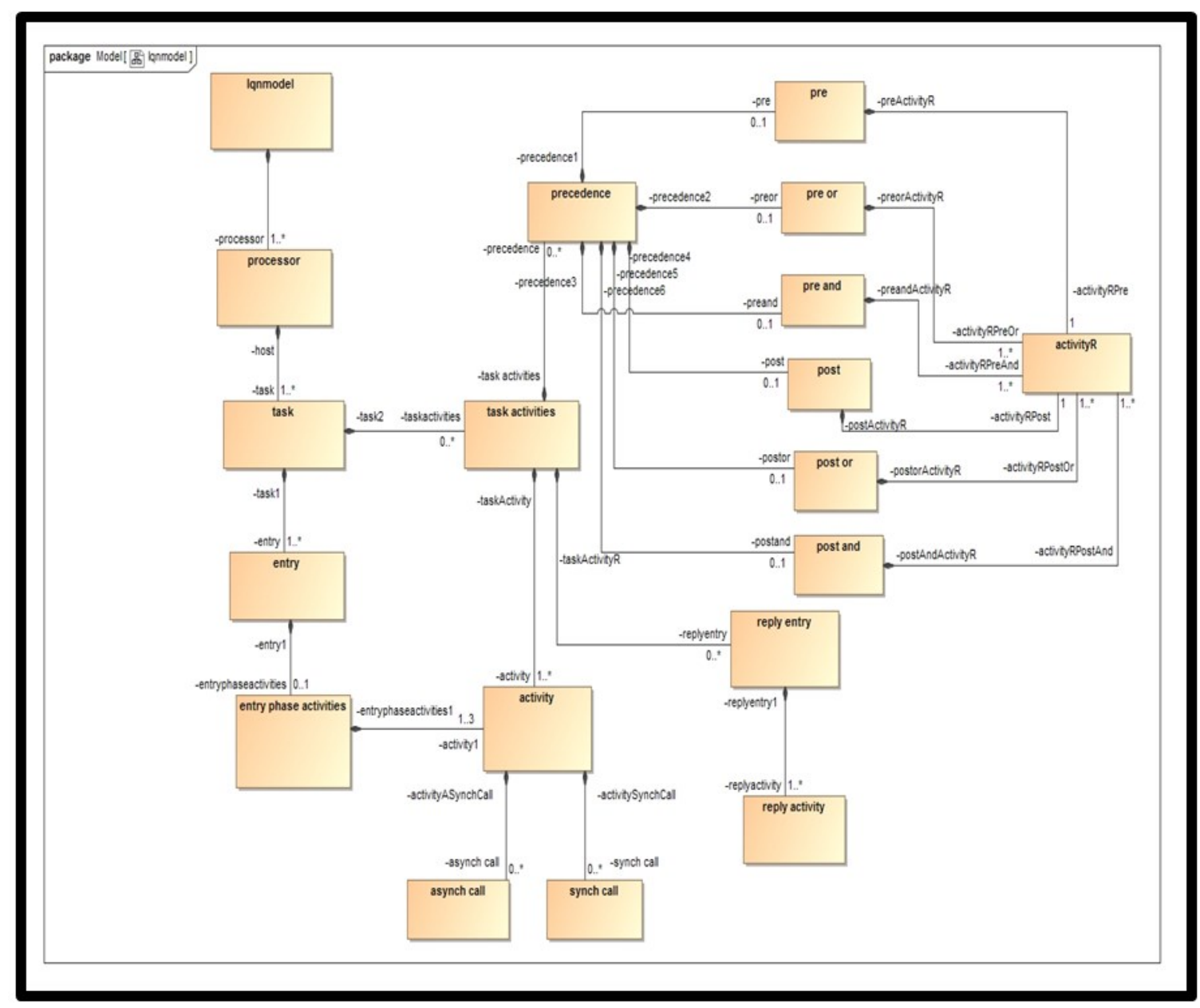

Figure 3-16. LQN metamodel designed for the Epsilon ETL transformation 
Like the XML-based metamodel, the root model element of the new metamodel is lqnmodel, which is composed of one or more processor model elements by using composition associations. In other words, lqnmodel and processor have a whole-part relationship, which mirrors the hierarchy from the XML-based metamodel. Processor is composed of tasks, which in turn is composed of entries or task-activities. Also, as in Figure 3-15, entry is the parent of entry-phase-activities model element, which is the container of activity model element. Activity is the parent of children of type synch-call and asynch-call. Task-activities element is composed of elements of three types: activity, precedence and reply-entry. Reply-entry is the parent of reply-activity. In addition, like in Figure 3-15, the elements named pre, pre-or, pre-and, post, post-or and post-and are all children of precedence model elements. However, unlike Fig 3-15, where all children of precedence are the parents of activity, in this new metamodel an extra model element called activity $R$ is defined to play the role of children of precedence. The reason for defining activity $R$ is that during the execution of the transformation, activity model elements are generated, which are either contained by entry-phase-activities or by taskactivites. In the case that their parent is task-activity, the same activity instance cannot be owned by other containers, such as precedence. ETL generates errors and stops the execution in this case. Therefore, a solution was to define a new model element called activityR to serve as child of precedence. Each activityR mirrors an activity element owned by task-activity and it is assigned the same attribute values in the transformation. Finally, in the post-transformation re-formatting step, activityR is replaced by activity in the LQN model. 


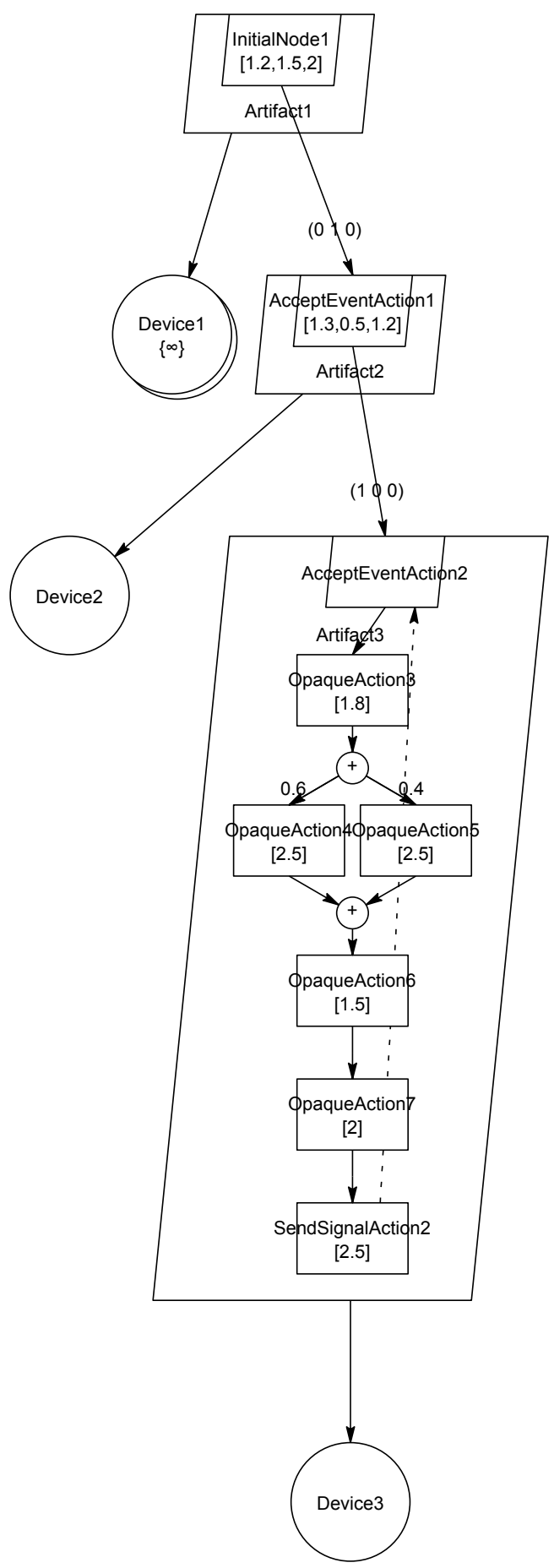

Figure 3-17. Graphical notation for the LQN model automatically generated from the UML model from Figures 3-1 and 3-2 
It has to be noted that the attributes of each model element and the enumeration types are not shown in this figure, but they appear in the Emfatic code. The Emfatic definition of the metamodel from Figure 3-16 is given in the Appendix A of the thesis.

Figure 3-17 shows an example of LQN model generated from the UML source model given in Figure 3-1 and Figure 3-2 by the transformation developed in the thesis. A description of how each model element of this figure is transformed and generated is given in Chapter 5. The XML format of the LQN model from Figure 3-17 is given in Appendix B in two formats: the "preliminary" LQN model generated by the Epsilon ETL model transformation is shown in appendix B1, and the actual LQN input model accepted by the LQN tools in Appendix B2. 


\section{Chapter: Transformation Design}

\subsection{Multi-step process for generating LQN models from UML+MARTE}

In order to automatically derive an LQN performance model from a UML+MARTE software model, we developed the multi-step process shown in Figure 4-1, which includes model transformation, model refining and model editing steps.

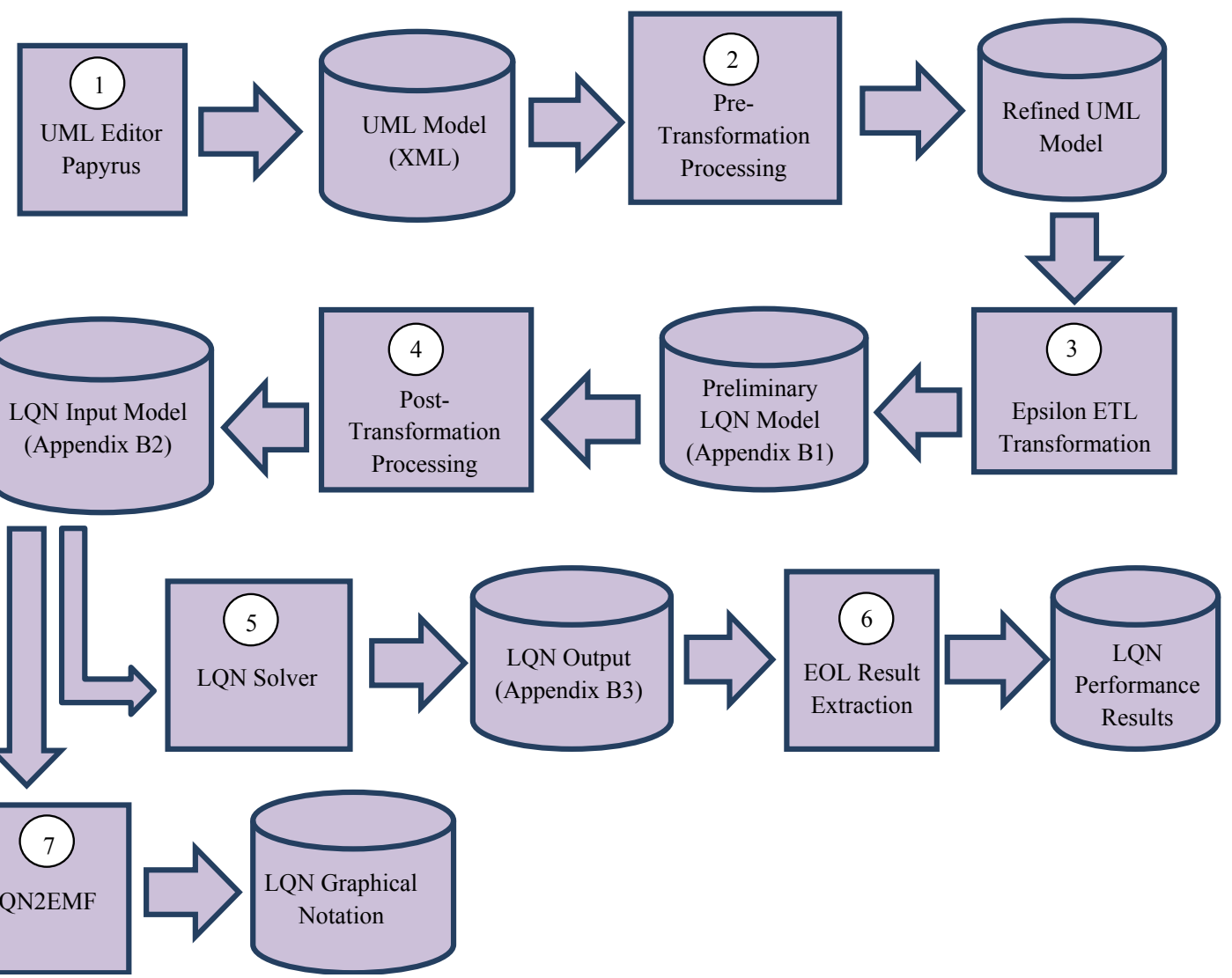

Figure 4-1. Multi-step process for deriving LQN models from UML+MARTE models

The steps of the process are as follows:

- Step 1: Building the source model, which is an UML software model with MARTE performance annotations, as described in Section 3.1. In our case, we used the opensource UML editor Papyrus [Papy15], whose development is supported by PolarSys [Pola16], an Eclipse Industry Working Group created by large industry players and 
by tools providers to collaborate on the creation and support of Open Source tools for the development of embedded systems.

- Step 2: Pre-transformation processing of the source model. This is an optional step for checking and refining the source model in order to discover and eliminate bugs or fix missing data before feeding it to the next step, the main model transformation. We are using this step for calculating derived attributes that are not set by Papyrus, as explained in Section 5.2. Other verifications can be included in the future, as for instance assigning default values to missing performance annotations.

- Step 3: Epsilon ETL transformation is the main step of the process. It bridges the big semantic gap between UML and LQN and generates the "preliminary" LQN model in XML format, which needs some further processing. (An example of such model is shown in Appendix B1).

- Step 5: Post-transformation processing makes minor edits in the preliminary XML file, which are needed because of the differences between the original LQN metamodel based on the XML schema [LQN15] and the LQN metamodel defined in Emfatic in the thesis (Appendix A). These differences were explained in section 3.2. Examples of changes performed in this step are as follows (described in more details in Section 5.3):

a) insert dashes '-' in identifiers as required by the original metamodel (Emphatic does not accept '-' in identifiers);

b) insert a solver-param element (which cannot be derived from the source model) at the beginning of the LQN model, before the starting tag of the processor element. 
c) replace the role names "activityR" and "activityl" (defined in the Emphatic metamodel) by "activity" (as used in the original LQN).

The generated LQN model can be parsed and solved by the existing LQN tools, and/or can be transformed in a different existing LQN format (graphical or textual).

- Step 5: Solving the LQN Model can be performed with one of the existing solvers (analytic or by simulation), which produces an LQN output file which includes performance results (elements shown in blue in Figure 3-15).

- Step 6: Extracting results values from the LQN output. The performance result values produced by the solver in the previous step can be individually extracted with the help of an EOL script (as described in section 5.4). Such results can be used for automatic diagnosis of performance problems, which is out of the thesis scope.

- Step 7: Transforming textual XML LQN file into graphical notation by using existing commands from the LQN toolset. The graphical notation is very useful for human users. An example of graphical notation obtained from XML is shown in Figure 3-17.

\subsection{UML+MARTE to LQN Mapping}

The main transformation represented by Step 3 consists of a set of transformation rules defined in more details in Chapter 5. As already mentioned, the input to the transformation is a UML model extended with the Performance Analysis Modeling (PAM) subprofile of MARTE. The source models must conform to the standard UML2.5 metamodel, whose definition is an Eclipse plugin imported by both the UML editor and the transformation tool. The stereotype attributes (represented by UML properties) and their values are referred to as Tag Definitions and tagged values respectively [UML15/p270] and must be conform to the standard MARTE definition [MARTE11]. 
The stereotypes and their properties add performance-related information to the input model. It is important to note that an input UML model has to be created based on the assumptions given in Section 3.1. If an input model does not satisfy these assumptions, the transformation will not execute properly.

The transformation is designed based on the mapping established by the designer between the model elements of the source and the target model. Figure 4-1 shows a highlevel view of some of the mapping between UML+MARTE model elements and LQN model elements. The mapping follows the hierarchical structure of the two models, as described in Chapter 3.

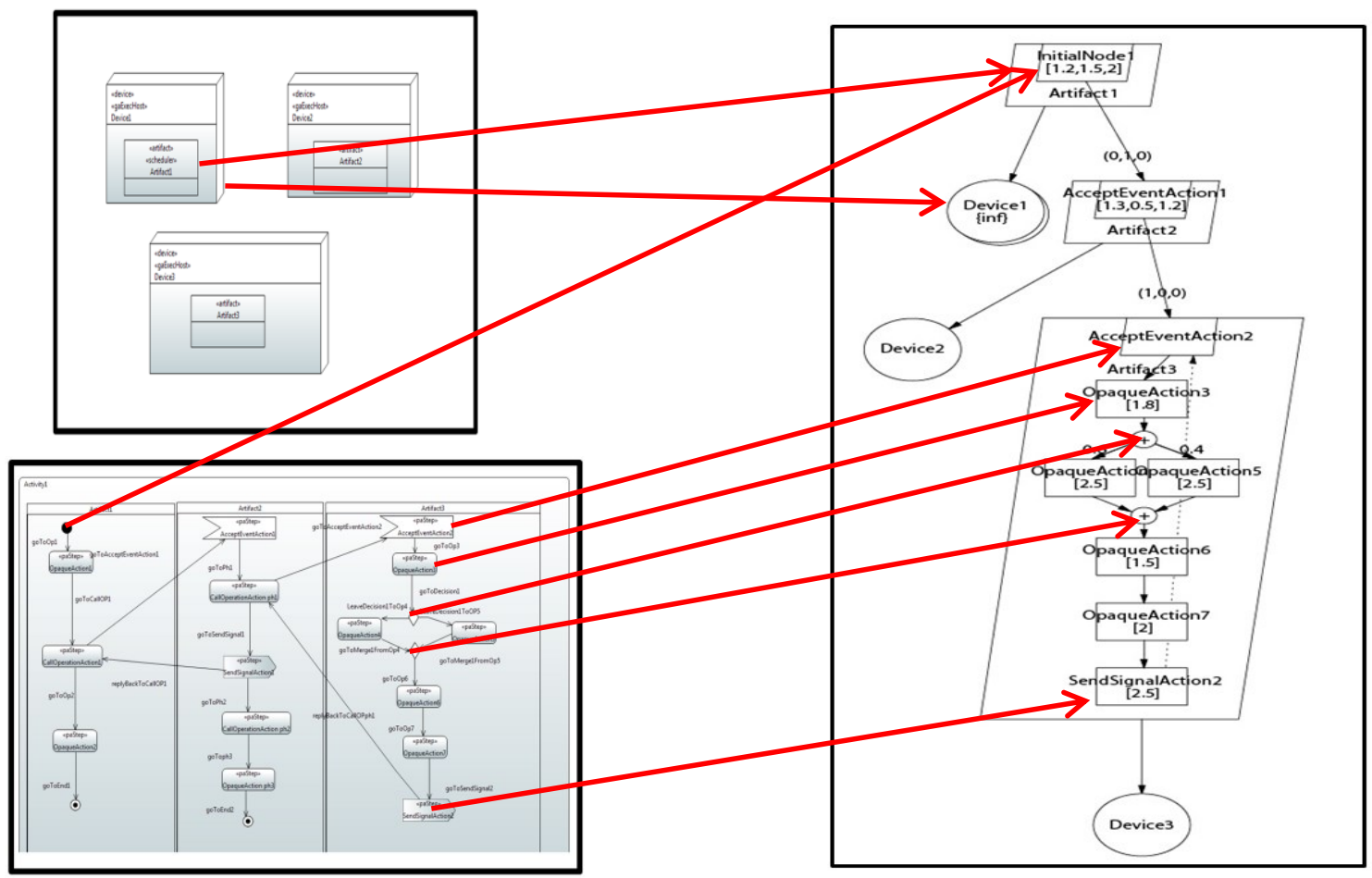

Figure 4-2. High-level view of the mapping between the source and target models

Table 4-1 shows all the mappings from the UML model elements annotated with some of the MARTE stereotypes to LQN model elements, represented by red arrows in the previous figure. This table contains three columns: the first gives the name of the UML 
model element, the second gives the stereotype which might have been applied to the UML element, and the third shows the corresponding LQN model element.

Table 4-1. Mapping from UML source to LQN target model

\begin{tabular}{|l|l|l|}
\hline UML Model Element & MARTE Stereotype & LQN Element \\
\hline Model & None & lqnmodel \\
\hline \multicolumn{2}{|c|}{ Deployment Diagram } \\
\hline Device & GaExecHost & processor \\
\hline Artifact & Scheduler & task \\
\hline \multicolumn{2}{|c|}{ Activity Diagram } \\
\hline AcceptEventAction & PaStep & entry \\
\hline InitialNode & None & entry \\
\hline OpaqueAction & PaStep & activity \\
\hline CallOperationAction & PaStep & activity \\
\hline SendSignalAction & PaStep & activity \\
\hline ControIFlow & None & precedence \\
\hline DecisionNode & None & precedence \\
\hline MergeNode & None & precedence \\
\hline JoinNode & None & precedence \\
\hline ForkNode & None & precedence \\
\hline ControlFlow & None & synch-call \\
\hline ControlFlow & None & asynch-call \\
\hline
\end{tabular}

\subsection{Transformation rules and operations overview}

The transformation is designed such that one ETL module contains all the transformation rules and operations corresponding to the mapping table given in Table 31. The rules show what LQN elements are to be created for the UML+MARTE elements discovered in the input model, and the operation are functions called by the transformations that verify some conditions or inspect the model and return some results. The transformation module contains one Matched Rule, 16 Lazy Rules, and 26 EOL operations. Table 4-2 and Table 4-3 give a brief description of the role of each ETL rule and operation, respectively. More elaborate definitions are given in Chapter 5.

Table 4-2. Transformation Rules for UML to LQN transformation

\begin{tabular}{|l|l|}
\hline Name & Description \\
\hline Model2Lqnmodel & Transforms UML Model to LQN Iqnmodel element \\
\hline
\end{tabular}




\begin{tabular}{|l|l|}
\hline Device2processor & Transforms UML Device to LQN processor element \\
\hline Artifact2task & Transforms UML Artifact to LQN task element \\
\hline AcceptEventAction2entry & $\begin{array}{l}\text { Transforms UML AcceptEventAction to LQN entry element } \\
\text { provided that the guard [not isNONEPattern()] is satisfied }\end{array}$ \\
\hline $\begin{array}{l}\text { AcceptEventAction2entry } \\
\text { None }\end{array}$ & $\begin{array}{l}\text { Transforms UML AcceptEventAction to LQN entry element } \\
\text { provided that the guard cond [isNONEPattern()] is satisfied }\end{array}$ \\
\hline InitialNode2entryPh1Ph2 & $\begin{array}{l}\text { Transforms UML InitialNode to LQN entry element provided } \\
\text { that the guard condition: not isNONEPattern() is satisfied }\end{array}$ \\
\hline InitialNode2entryNone & $\begin{array}{l}\text { Transforms UML InitialNode to LQN entry element provided } \\
\text { that the guard condition [isNONEPattern()] is satisfied }\end{array}$ \\
\hline OpaqueAction2Activity & Transforms UML OpaqueAction to LQN activity element \\
\hline DecisionNode2Precedence & Transforms UML DecisionNode to LQN precedence element \\
\hline ControlFlow2Precedence & $\begin{array}{l}\text { Transforms UML ControlFlow to LQN precedence element } \\
\text { provided that the guard condition [guardForControlFlowTo } \\
\text { Precedence()] is satisfied }\end{array}$ \\
\hline MergeNode2Precedence & Transforms UML MergeNode to LQN precedence element \\
\hline JoinNode2Precedence & Transforms UML JoinNode to LQN precedence element \\
\hline ForkNode2Precedence & Transforms UML ForkNode to LQN precedence element \\
\hline CallOperationAction2Activity & Transforms UML CallOperationAction to LQN activity element \\
\hline CondSignalAction2Activity & Transforms UML SendSignalAction to LQN activity element \\
\hline ControlFlow2asynchcall & Transforms UML ControlFlow to LQN synchcall element \\
\hline
\end{tabular}

As shown in Table 4-2, two kinds of ETL rules (Matched rule and Lazy rule) are used for this transformation. In ETL, Matched rules are the ones that are executed automatically by the engine when it finds an element in the source model which matches with the source element from the rule. Lazy rules, instead, are invoked explicitly in other rules by using the ETL built-in equivalent() operation. Epsilon ETL is a hybrid language, where Matched rules correspond to the declarative style, while Lazy rules corresponds to the imperative style. 
Within each rule, the logic for connecting each generated target element with another is defined. Target elements are linked together by using containment association relationships. For example, our transformation starts with the execution of the only Matched rule, Model2Lqnmodel, to transform the root element (UML Model) of the source model to the root element ( $L Q N$ lqnmodel) of the target model. Since the target root element has a containment relationship with the $L Q N$ processor element, the generated $L Q N$ processor has to be added as a Child to the target root element. Thus the matched rule Model2Lqnmodel invokes the Lazy rule Device2processor to generate a target processor for each processor element found in the input model, and then adds the newly created target element to the corresponding container of LQN lqnmodel.

In turn, Rule Device2processor invokes Artifact2task rule. Rule Artifact2task invokes one of the four rules which transform either a UML AcceptEventAction or a $U M L$ InitialNode to an element of $L Q N$ entry type. It also transforms different kinds of $U M L$ ActivityNode, such as OpaqueAction, CallOperationAction and SendSignalAction to the $L Q N$ activity type by invoking their corresponding mapping rules. It also invokes the rules which generate $L Q N$ precedence from $U M L$ ControlFlow and some of the $U M L$ ControlNode, such as DecisionNode, MergeNode, JoinNode and ForkNode. The rule generating an $L Q N$ activity from a source element of type UML CallOperationAction invokes one of the two rules generating $L Q N$ synchcall or $L Q N$ asynchcall from a $U M L$ ControlFlow.

Table 4-3. Operations in UML to LQN transformation

\begin{tabular}{|l|l|}
\hline Name & Description \\
\hline checkPSchedulerType & $\begin{array}{l}\text { For the given processor's scheduling policy (otherSchedPolicy), } \\
\text { returns its corresponding value of LQN enumeration processor } \\
\text { scheduling type }\end{array}$ \\
\hline
\end{tabular}




\begin{tabular}{|c|c|}
\hline checkTSchedulerType & $\begin{array}{l}\text { For the given task's scheduling policy (otherSchedPolicy), } \\
\text { returns its corresponding value of LQN enumeration task } \\
\text { scheduling type }\end{array}$ \\
\hline getStereotypeOfDevice & $\begin{array}{l}\text { For the given UML Device, returns the String value of } \\
\text { otherSchedPolicy property of its GaExecHost stereotype }\end{array}$ \\
\hline getStereotypeOfArtifact & $\begin{array}{l}\text { For the given UML Artifact,returns the String value of } \\
\text { otherSchedPolicy property of its Scheduler stereotype }\end{array}$ \\
\hline ReturnProbability & $\begin{array}{l}\text { For the given UML Action defined in Activity Digram, returns the } \\
\text { Real value of prop property of its PaStep stereotype }\end{array}$ \\
\hline $\begin{array}{l}\text { FindlnitialNodeOrAcceptEv } \\
\text { entActionBackwardsInThe } \\
\text { Flow }\end{array}$ & $\begin{array}{l}\text { For the given UML ControlFlow and its ActivityPartition, returns } \\
\text { either InitialNode or AcceptEventAction as an origin of the activity } \\
\text { graph located in the given ActivityPartition by traversing it } \\
\text { backward recursively }\end{array}$ \\
\hline $\begin{array}{l}\text { TraverseTheGraphForward } \\
\text { ToTheEnd }\end{array}$ & $\begin{array}{l}\text { For the given UML ControlFlow and its ActivityPartition, returns } \\
\text { three collections of ActivityNodes, ControlFlows and } \\
\text { ControlNodes by traversing Activity graph located in the given } \\
\text { ActivityPartition forward recursively }\end{array}$ \\
\hline isNONEPattern & $\begin{array}{l}\text { For the given UML ControlFlow, determines if the type of Activity } \\
\text { graph is NONE, and returns a Boolean value }\end{array}$ \\
\hline $\begin{array}{l}\text { guardForSendSignalAction } \\
\text { 2Activity }\end{array}$ & $\begin{array}{l}\text { For the given UML ControlFlow, determines if the ControlFlow } \\
\text { and its target SendSignalAction are located in the Activity graph, } \\
\text { which its type is NONE, and returns a Boolean value }\end{array}$ \\
\hline $\begin{array}{l}\text { guardForControlFlowTo } \\
\text { Precedence }\end{array}$ & $\begin{array}{l}\text { For the given UML ControlFlow, determines if the ControlFlow is } \\
\text { located in the Activity graph, which its type is NONE and, also } \\
\text { satisfies the conditions for being sequential, and returns a } \\
\text { Boolean value }\end{array}$ \\
\hline isSequential & $\begin{array}{l}\text { For the given UML ControlFlow, determines if the conditions for } \\
\text { being sequential are met, and returns a Boolean value }\end{array}$ \\
\hline EntryFunctionOperation & $\begin{array}{l}\text { For the given UML InitialNode or AcceptEventAction, their } \\
\text { ActivityPartions, and the generated LQN task corresponding to } \\
\text { the UML Artifact, invokes ETL built-in equivalent() operation to } \\
\text { generate LQN entries and precedence ,and to instantiate LQN } \\
\text { taskactivities, entryphaseactivities, replyentry, and replyactivity }\end{array}$ \\
\hline $\begin{array}{l}\text { getoutgoingofthisCallOpera } \\
\text { tionActionwhichTargetlsAc } \\
\text { ceptEventAction }\end{array}$ & $\begin{array}{l}\text { For the given UML CallOperationAction, checks for its outgoing } \\
\text {,which its target type is AcceptEventAction, and returns that } \\
\text { outgoing }\end{array}$ \\
\hline ToFindAndReturnSendSig & For the given UML ControlFlow and its ActivityPartition, returns \\
\hline
\end{tabular}




\begin{tabular}{|c|c|}
\hline nalAction & $\begin{array}{l}\text { SendSignalAction, which is part of the Activity graph located in } \\
\text { given ActivityPartition by traversing it forward recursively }\end{array}$ \\
\hline $\begin{array}{l}\text { controlflowProcessingfor } \\
\text { ASynchCall }\end{array}$ & $\begin{array}{l}\text { For the given UML ControlFlow, determines if it follows the } \\
\text { pattern for the Asynchronous call, and returns a Boolean value }\end{array}$ \\
\hline $\begin{array}{l}\text { controlflowProcessingfor } \\
\text { SynchCall }\end{array}$ & $\begin{array}{l}\text { For the given UML ControlFlow, determines if it follows the } \\
\text { pattern for the Synchronous call, and returns a Boolean value }\end{array}$ \\
\hline $\begin{array}{l}\text { controlflowProcessingfor } \\
\text { Branch }\end{array}$ & $\begin{array}{l}\text { For the given UML ControlFlow, determines if its target is type of } \\
\text { UML DecisionNode, and returns Boolean value }\end{array}$ \\
\hline $\begin{array}{l}\text { controlflowProcessingfor } \\
\text { Merge }\end{array}$ & $\begin{array}{l}\text { For the given UML ControlFlow, determines if its source is type } \\
\text { of UML MergeNode, and returns Boolean value }\end{array}$ \\
\hline $\begin{array}{l}\text { controlflowProcessingfor } \\
\text { Fork }\end{array}$ & $\begin{array}{l}\text { For the given UML ControlFlow, determines if its target is type of } \\
\text { UML ForkNode, and returns Boolean value }\end{array}$ \\
\hline $\begin{array}{l}\text { controlflowProcessingfor } \\
\text { Join }\end{array}$ & $\begin{array}{l}\text { For the given UML ControlFlow, determines if its source is type } \\
\text { of UML JoinNode, and returns Boolean value }\end{array}$ \\
\hline IsThisEdgelnThisPartition & $\begin{array}{l}\text { For the given UML ControlFlow and ActivityPartition, checks for } \\
\text { the edge of the ActivityPartion, Which has the same name as } \\
\text { the given ControlFlow }\end{array}$ \\
\hline getDevices & $\begin{array}{l}\text { For the given UML Model, checks for all the UML Devices, which } \\
\text { their namespace is the Model and are annotated by GaExecHost } \\
\text { stereotype, and returns a collection of those Devices }\end{array}$ \\
\hline getArtifact & $\begin{array}{l}\text { For the given UML Device, checks for all the UML Artifacts, } \\
\text { which their namespace is the Device, and returns a collection of } \\
\text { those Artifacts }\end{array}$ \\
\hline getActivityPartitions & $\begin{array}{l}\text { For the given Artifactname typed String, checks for the UML } \\
\text { ActivityPartitions, which their name is the same as the given } \\
\text { Artifactname, and returns a collection of those ActivityPartitions }\end{array}$ \\
\hline getActivitynodes & $\begin{array}{l}\text { For the given UML ActivityPartion, ckecks for its nodes, which } \\
\text { are kinds of UML ActivityNode, and returns a collection of those } \\
\text { ActivityNodes }\end{array}$ \\
\hline hasStereotype & $\begin{array}{l}\text { For the given name typed String and UML Class, determines if } \\
\text { stereotype's name applied on the Class is the same as the given } \\
\text { name, and returns a Boolean value }\end{array}$ \\
\hline
\end{tabular}




\section{Chapter: Transformation Implementation}

This chapter presents in details the transformation rules listed in Table 4-1, and some of the operations listed in Table 4-2.

\subsection{Rules and Operations}

\subsubsection{Rule Model2Lqnmodel}

The "lqnmodel" element is the top element of our target LQN model. This element, which is the root container of the target model, corresponds to the UML "Model" element. As we can see from the Code Fragment 5-1, the UML "Model" element, which is the root container of the source model, and indicated in section "transform", is transformed into the top "lqnmodel" element of a LQN.

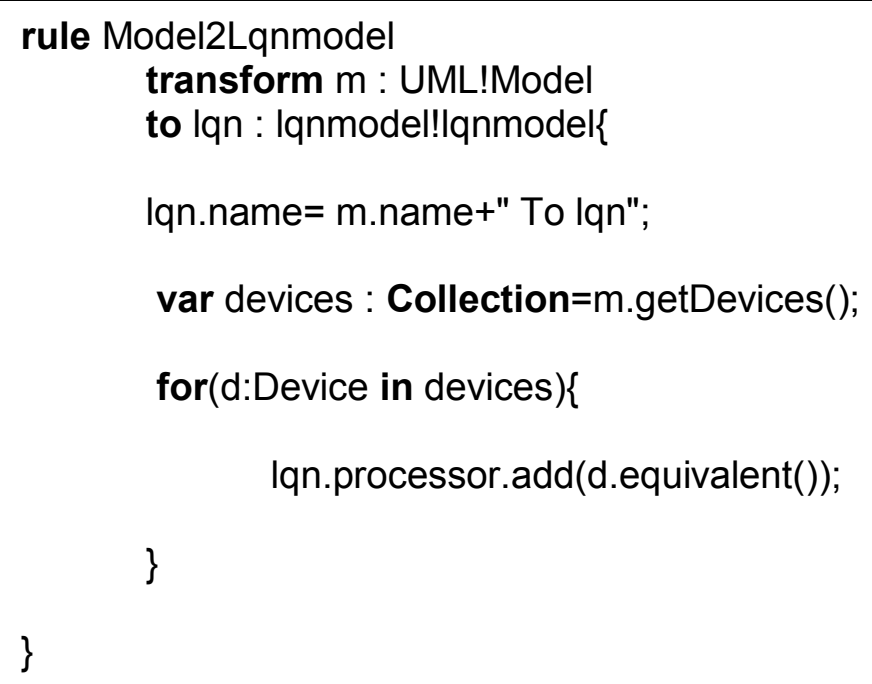

Code Fragment 5-1. Transformation rule Model2Lqnmodel

The rule shown in Code Fragment 5-1 contains one target component in section "to", which is labeled with the variable "lqn". This variable represents a generated target node of type "lqnmodel". The assignment statement involving the target node "lqn" (lqn.name= m.name+" To lqn") indicates how the "lqn.name" attribute is initialized with 
the name of the source "Model" element to which the string "To lqn" is appended. The operation "getDevices()" is invoked on the variable " $\mathrm{m}$ " that represents the "Model" element of the source model to return the collection of "Device" elements included in "Model". For each Device element in the collection, the call to "d.equivalent()" returns the LQN model element (a "processor") transformed from "d", which is then added to the collection "lqn.processor". The built-in ETL "equivalent()" operation resolves target elements that have been transformed from source elements by other rules [Kol15].

\subsubsection{Operation getDevices}

As mentioned above, this operation is called in the context of the source element "Model" and returns a collection of all the instances of type "Device" that have been stereotyped by the MARTE "GaExecHost" stereotype, and have their "namespace" attribute assigned to "Model". This operation is shown in Code Fragment 5-2. "GaExecHost" stereotype represents an execution resource [MART11]. In performance modeling, a GaExecHost can be any device that executes behavior as a processor, storage and peripheral [MART11]. Figure 5-1 shows an example of how the properties of a "Device" element of the source model have, named "Devicel" are set. The "Devicel" instance is stereotyped with "GaExecHost". Its property "namespace" needs to be initialized to the root element named "model" of type "Model".

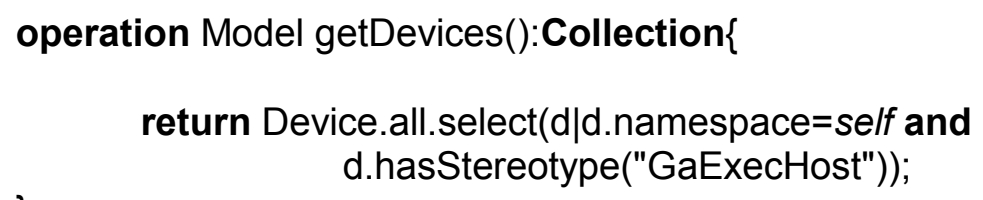

Code Fragment 5-2. Operation getDevices 


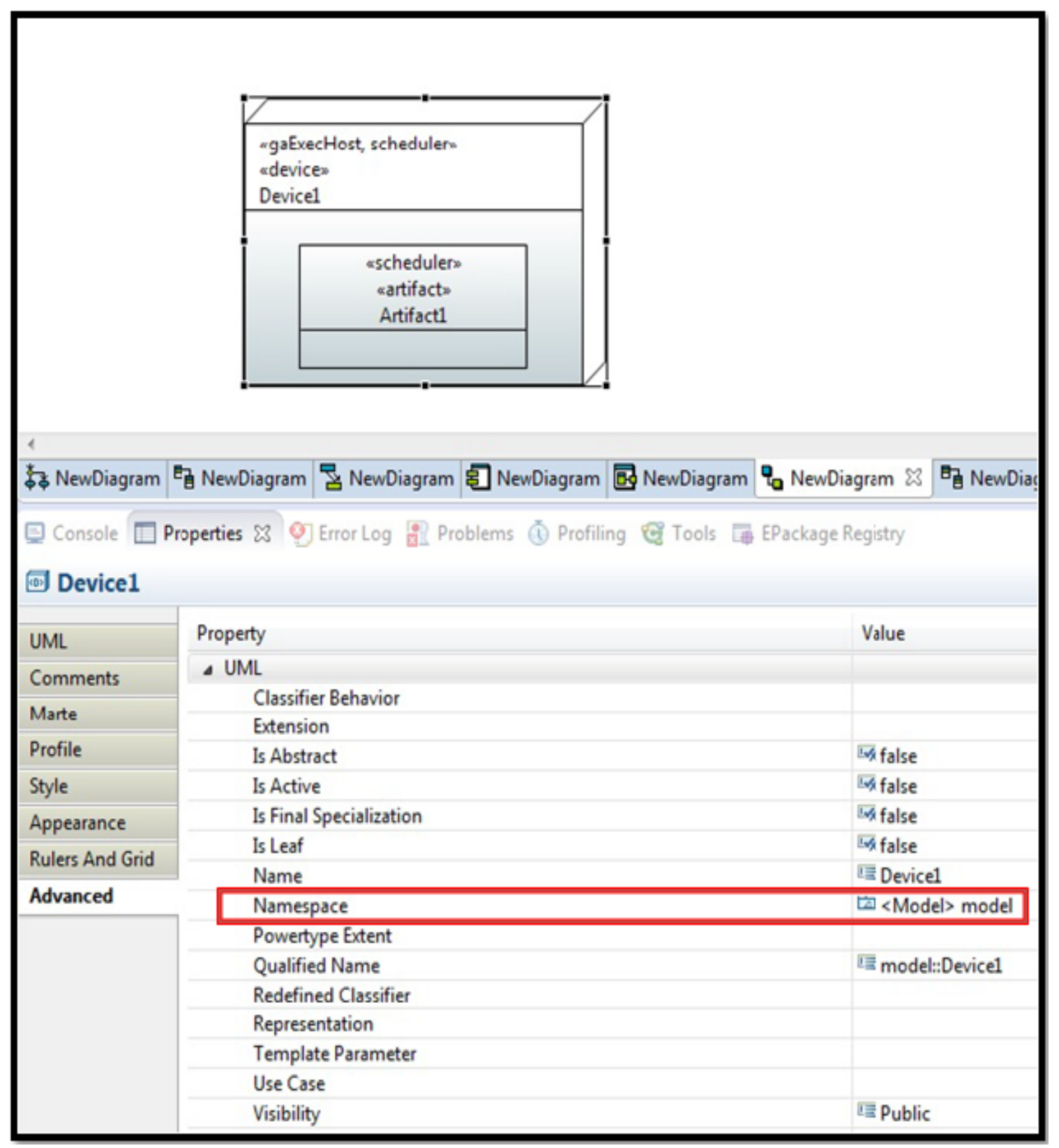

Figure 5-1. Example of "Device" Element of the Source Model

\subsubsection{Operation hasStereotype}

This operation is called in the context of a "Class" of the source model and returns a Boolean type value. As we can see in the Code Fragment 5-3, "getAppliedStereotypes()", an ETL built-in method, is invoked on the "self" variable which refers to the context of this operation. This method returns a sequence containing 
all the stereotypes that have been applied to the respective "Class". For each Stereotype instance in this collection, if its name attribute is the same as the String parameter passed as an argument to this operation, the operation will return "true".

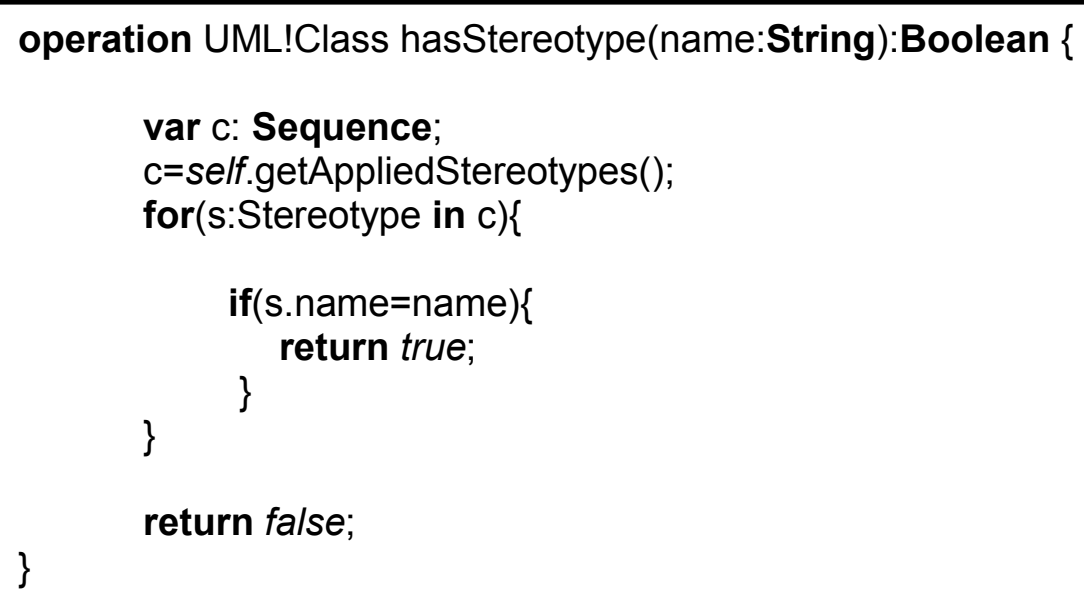

Code Fragment 5-3. Operation hasStereotype

\subsubsection{Rule Device2processor}

Each "Device" element from the deployment diagram of the source model is mapped to a "processor" element of the target LQN model, as depicted in Figure 5-2 and Code Fragment 5-4. The "guard" section of this rule is checking for the "Device" instances stereotyped with "GaExecHost".

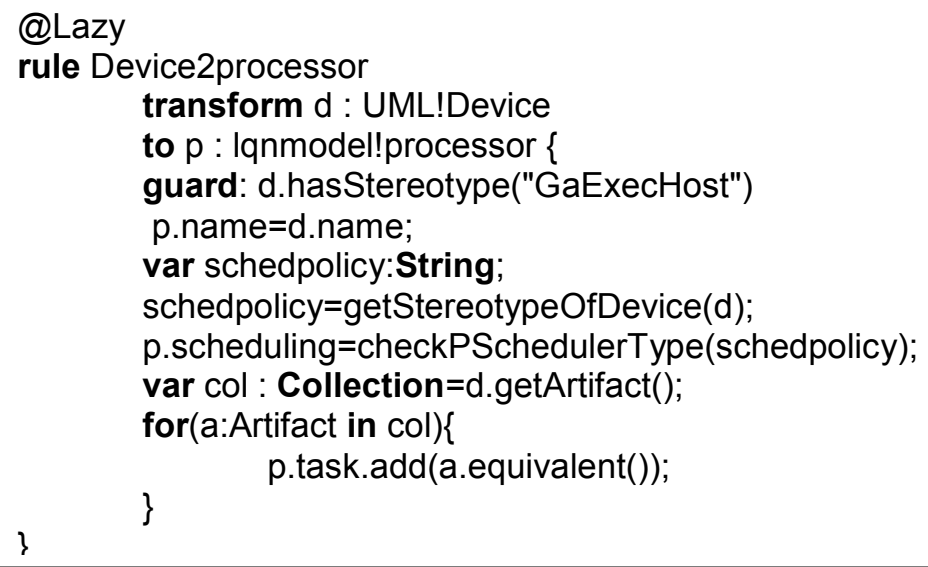

Code Fragment 5-4.Transformation rule - Device2processor 
For example, if there are "Device" instances in the source model which are not stereotyped with "GaExacHost", they will be excluded from the transformation rule. Transformation rules which include a "guard" section will be applicable only to the elements that satisfy the guard's condition. In this transformation, the name of a target "processor" will be initialized to the name of its corresponding source "Device". The method "getStereotypeofDevice()" which passes "Device" of the source model as a parameter, checks for the Stereotype "Scheduler". "Scheduler" stereotype represents a kind of ResourceBroker, which creates access to its brokered ProcessingResource or resources which follow a certain scheduling policy [MARTE11]. If this Stereotype is defined on the "Device" instance and its attribute "otherSchedPolicy" is set, the method returns the value of this attribute.

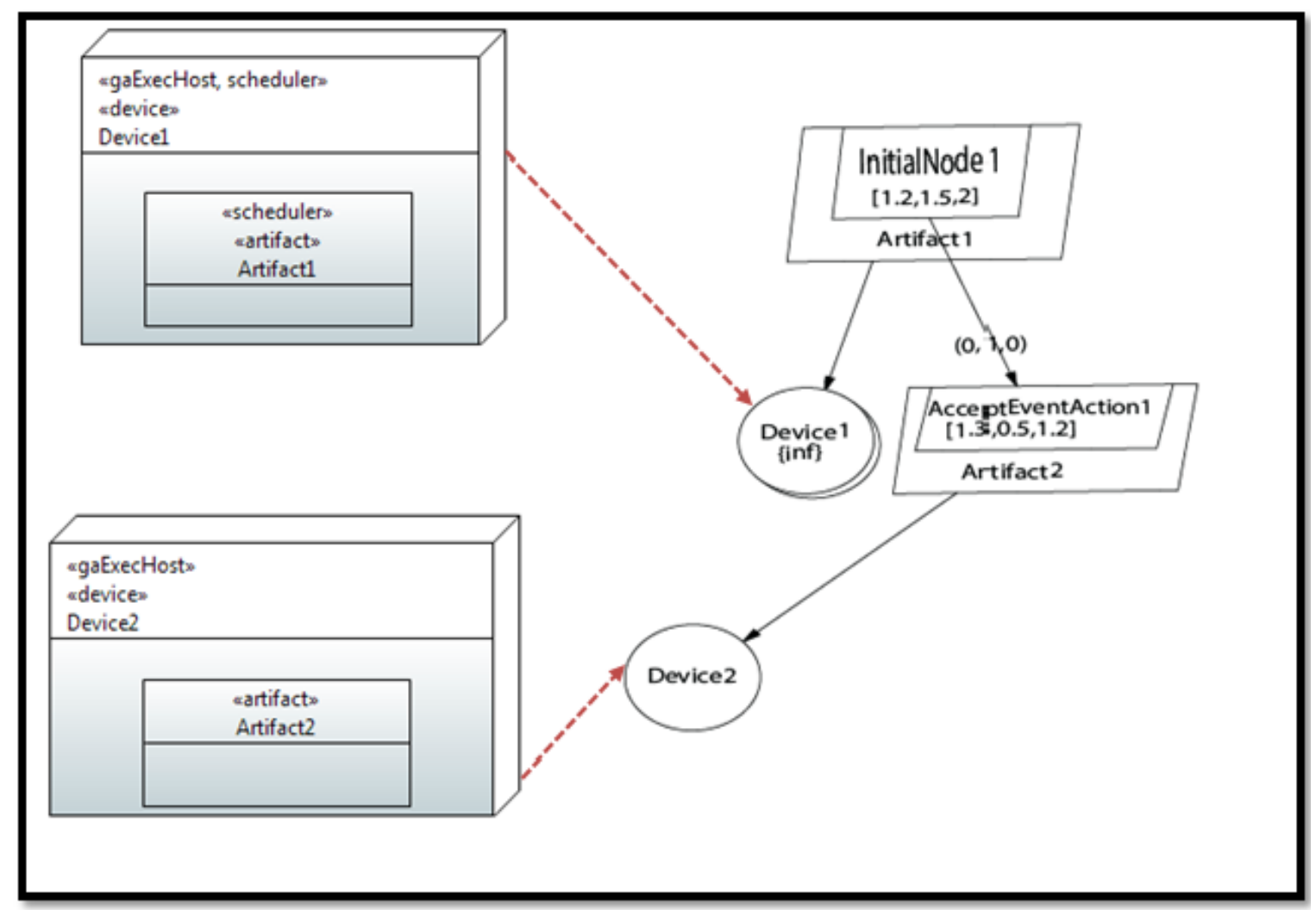

Figure 5-2. Transformation rule example - Device2processor 


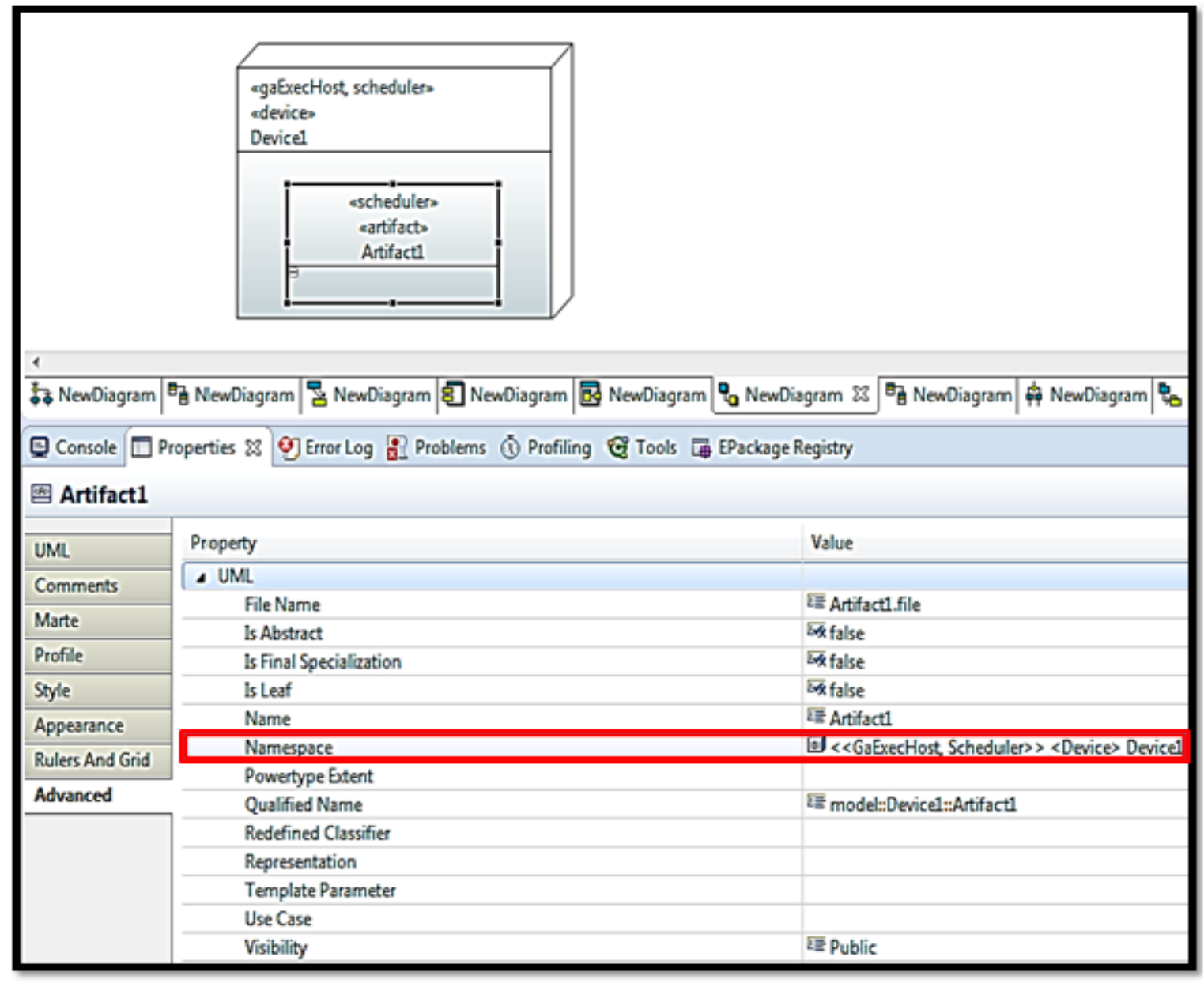

Figure 5-3. Example of "Artifact" Element of the Source Model

Using this value as a parameter to "checkPSchedulerType()" method, it sets the "scheduling" property of the "processor". "otherSchedPolicy" is one of the properties of the "Scheduler" stereotype, which annotates a scheduling policy not included among the values of the "schedPolicyKind" enumeration type [MART11]. It could be set for the object "processor" when creating the source model. Afterwards, there is "getArtifact()", which is called on an instance of "Device" and collects all the instances of the "Artifact" whose "namespace" attribute is set to the respective "Device". Figure 5-3 shows an example of how to set an "Artifact" element in the source model. For each Artifact element in the collection, its corresponding target "Task" element generated by a 
different transformation rule will be added to the "p.task" reference of the "processor" type instance from the target.

\subsubsection{Rule Artifact2task}

As we can see from Figure 5-4 and Code Fragment 5-5, each "Artifact" element (representing a software component) is transformed to a "task" element of the target LQN model. In this rule, after initializing the attributes "name" and "scheduling" of the "task" element, the "getActivityPartitions()" method is invoked. This method passes the name of the "Artifact" and returns a collection of all "ActivityPartition" instances whose names match this parameter. Figure 5-5 shows an example of how the "name" property of an "ActivityPartition" needs to be set when creating a UML activity diagram. In this collection, there are "ActivityPartition" elements with the same name as an "Artifact" from the deployment diagram. The method "getactivitynodes()" uses each element of this

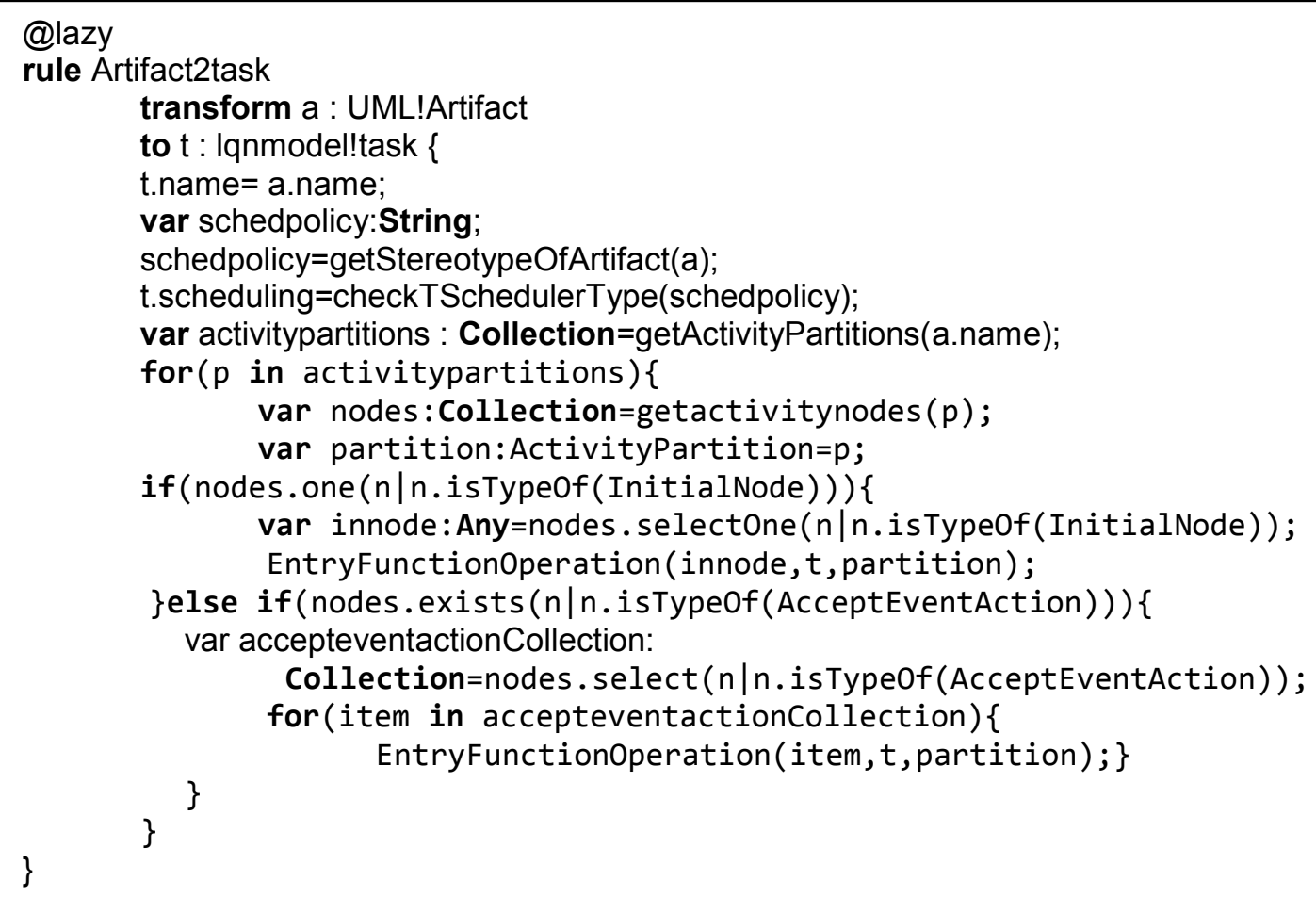

Code Fragment 5-5. Transformation rule - Artifact2Task 
collection to return a collection of all the "node" elements which of type "ActivityNode". (Please note that "ActivityNode" is an abstract type with many subtypes). If there exists exactly one "ActivityNode" element in this collection of concrete type "InitialNode", its corresponding target "task" represents a "Reference Task" in LQN; however, if there exists at least one "ActivityNode" object in this collection of concrete type "AcceptEventAction", its corresponding target "task" becomes a normal task. Reference tasks represent customers in the layered queueing network that drive the system; each has a single entry that makes requests, but never accept any requests from other tasks. They are always at the top of a call graph [LQN15]. In the case of a reference task, the "InitialNode" along with its "ActivityPartition" and target "task" are passed to "EntryFunctionOperation()" method. Otherwise, in the case of a normal task, the "EntryFunctionOperation()" method is invoked for each element of “AcceptEventAction" found.

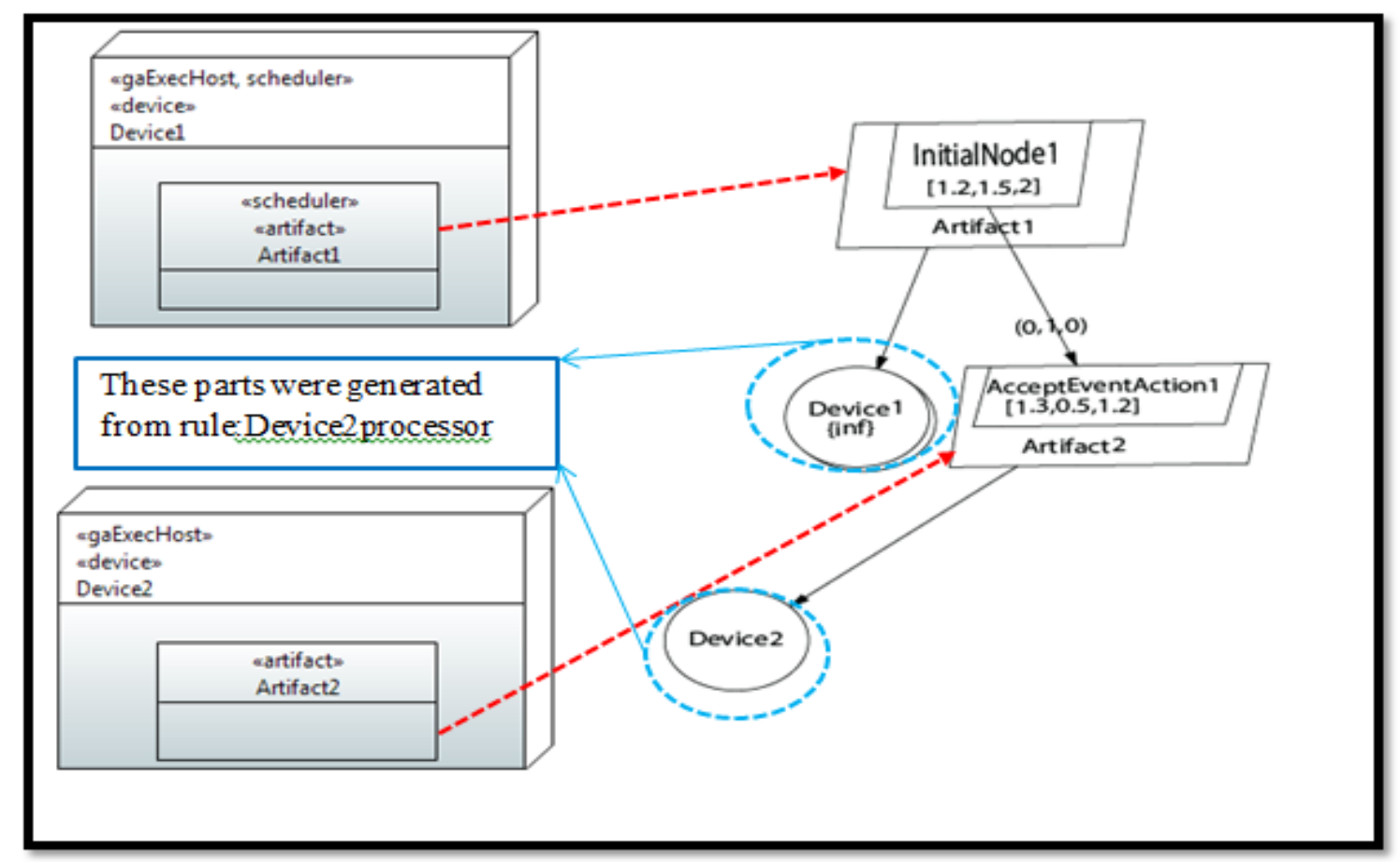


Figure 5-4. Transformation rule example - Artifact2task

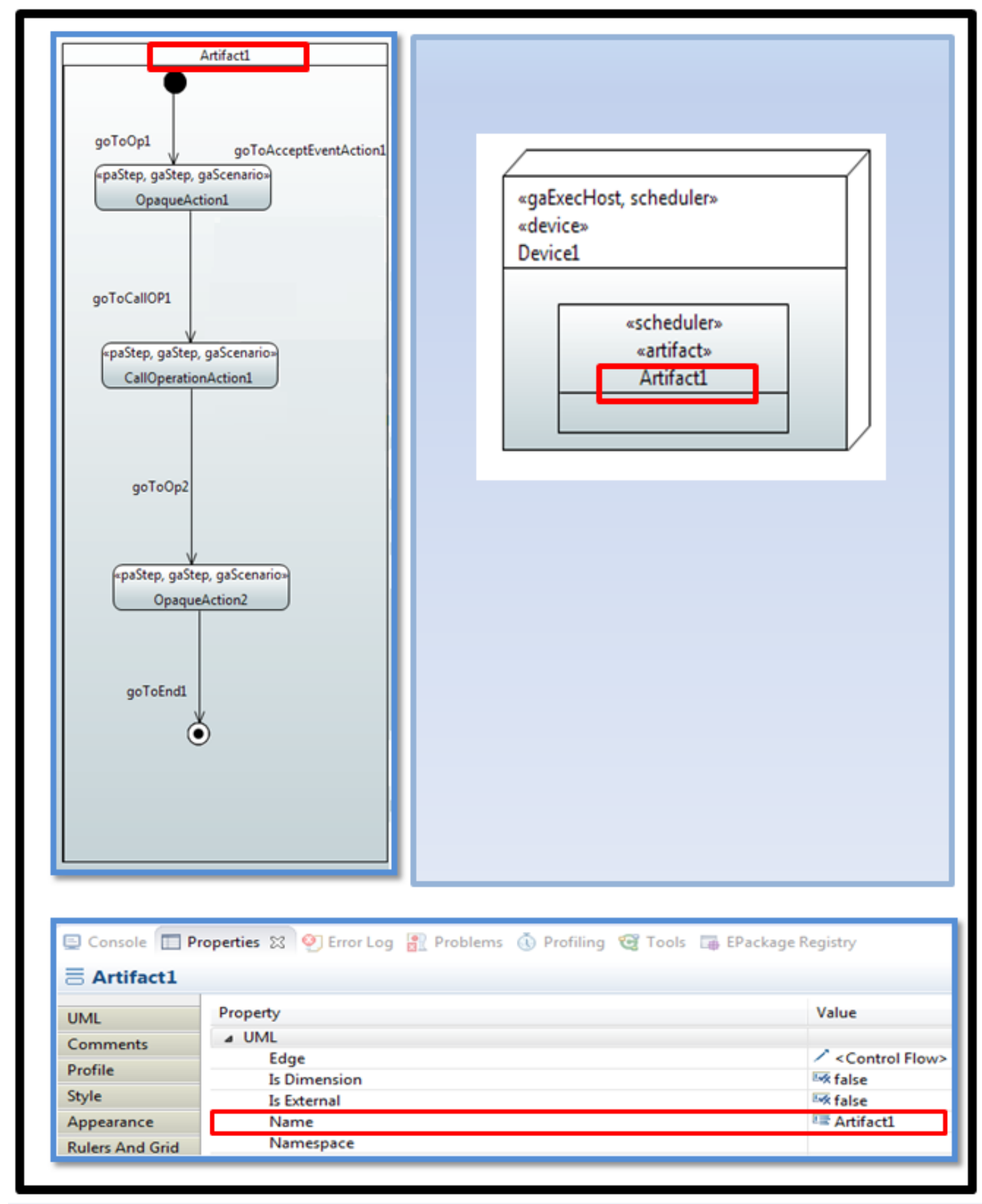

Figure 5-5. Example of "ActivityPartition” Element of the Source Model

This operation which is given either "InitialNode" or "AcceptEventAction" as its first parameter, invokes the "equivalent()" ETL built-in operation on this parameter in order to 
create their corresponding target "entry". If attribute "type" of this entry is "NONE", it traverses the graph starting with "InitialNode" or "AcceptEventAction" to its end in order to gather collections of "ControlFlow", "ControlNode" and "ActivityNode", repectively. Besides, LQN "taskactivities" is instantiated to hold LQN "activity" objects, which are created for each UML "ActivityNode" in the collection gathered above. (Each LQN "activity is found by the "equivalent()" operation invoked on the collection of "ActivityNode"). If there exists in this collection at least one item of type "SendSignalAction" (which sends the reply back to a CallOperationAction), LQN "replyentry" and "replyactivity" will be instantiated respectively in the target model. For each item from the "ControlNode" collection, various conditions need to be checked in order to transform either the item itself, its source or its target to the corresponding LQN elements by invoking the ETL "equivalent()" operation. For instance, if the target property of the "ControlFlow" is type of UML "DecisionNode", this object will be transformed to LQN "precedence".

Now let us consider that the property "type" of "entry" is "PHPH2". In this case, like before, after traversing the activity graph and collecting the required collections, some elements such as "SendSignalAction" and "ActivityFinalNode" will be excluded from the "ActivityNode" collection before transforming the content of this collection, because in the case of "PHPH2" there are no equivalent LQN elements in the target model. However they have to be depicted in the UML source model to be used by other transformation methods and rules for other analysis purposes. Finally LQN "entryphaseactivities" is instantiated to comprise LQN "activity" instances transformed from the content of the "ActivityNode" collection. It is important to mention that for each "ControlFlow" object 
located inside an "ActivityPartition" (meaning that it does not cross the partition's border), its property of "inPartition" has to be set manually in the source model before running the transformation, as in shown in Figure 5-6. For the border crossing "ControlFlow", this feature does not have to be set.

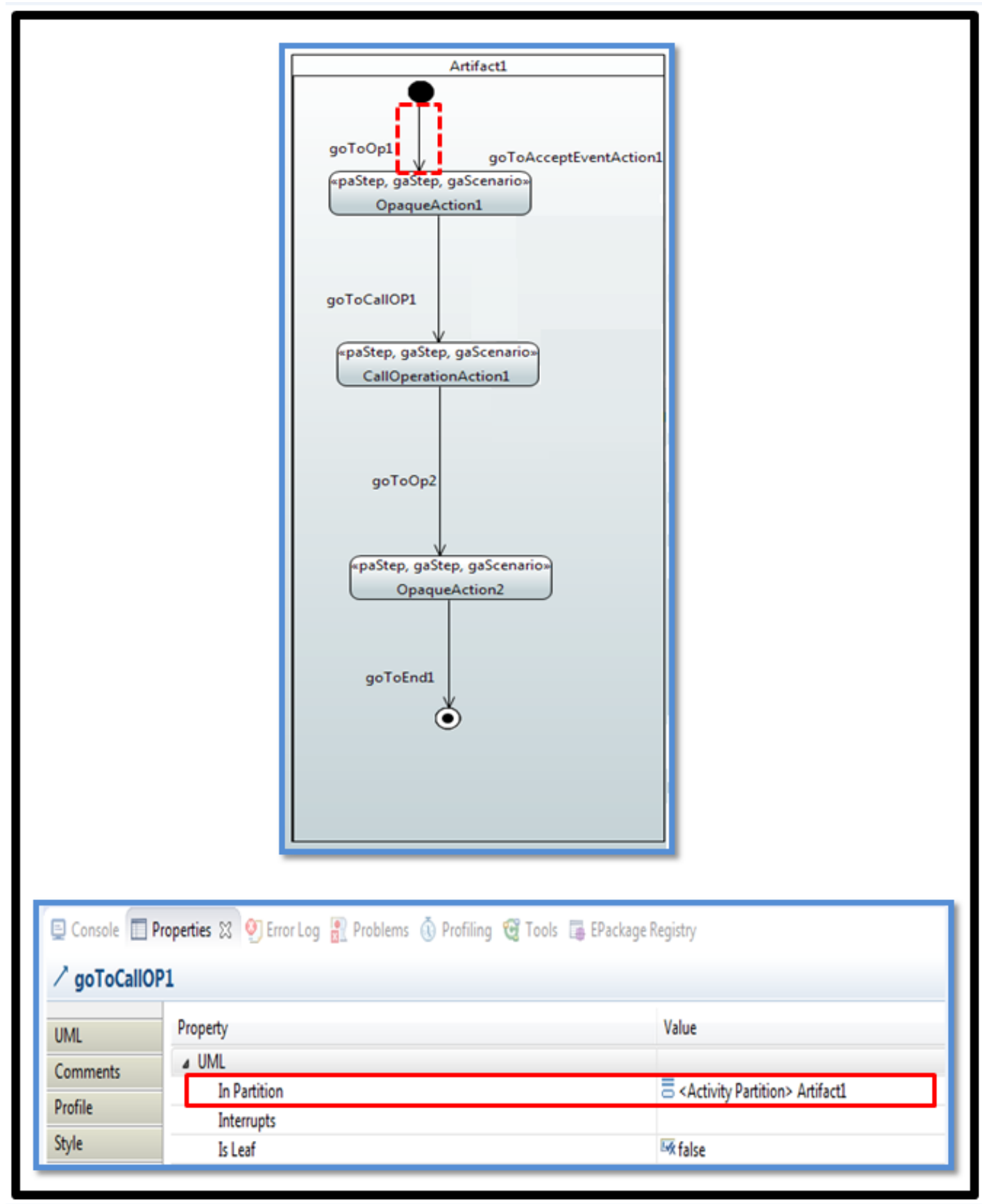

Figure 5-6. Example of "ControlFlow" Element of the Source Model 


\subsubsection{Rule InitialNode2entryPh1Ph2}

Within each "ActivityPartition", as shown in Figure 5-7 and Code Fragment 5-6, each "InitialNode" will be transformed into an LQN "entry", provided that its "guard" condition is satisfied. Operation "isNONEPattern()", which is a part of the guard's condition, takes a "ControlFlow" instance as an argument and returns a "Boolean" type value. Using the logical operator "not", evaluates the logical negation of this operation. If the negation of this expression is "true", the guard's condition is satisfied, and "InitialNode" will be transformed to an LQN "entry" and its attribute "type" will be set to "PH1PH2" ( a value of enumeration "EntryType"). Let consider the example from Figure 5-7, where a "ControlFlow" object named "goToOp1", outgoing from the "InitialNode", is passed to the operation "isNONEPattern()". The operation starts at "goToOp1" and traverses the graph upward until it reaches the origin of the flow, which in general is either "InitialNode" (as in Figure 5-7) or "AcceptEventAction" (in other cases). Using the origin, it starts traversing the graph downward until reaching the end of the flow; in Figure 5-7, the end is the "ActivityFinalNode". The graph traversal results in collecting three collections of elements contained in the partition: "ControlFlow", "ControlNode" and "ActivityNode". Then from "ActivityNode" collection, some elements such as "SendSignalAction" and "ActivityFinalNode", which are not required for generating an entry of type PH1PH2, will be removed. The analysis of these collections shows whether the conditions for the entry pattern "PH1PH2" are met or not. The pattern describes entries with one, two or three activities in sequence (the activities are called "phases" in this case), where the reply to the caller is sent after the first activity (or phase 1). Any other configuration of the activity graph leads to the pattern "NONE" for the entry. 


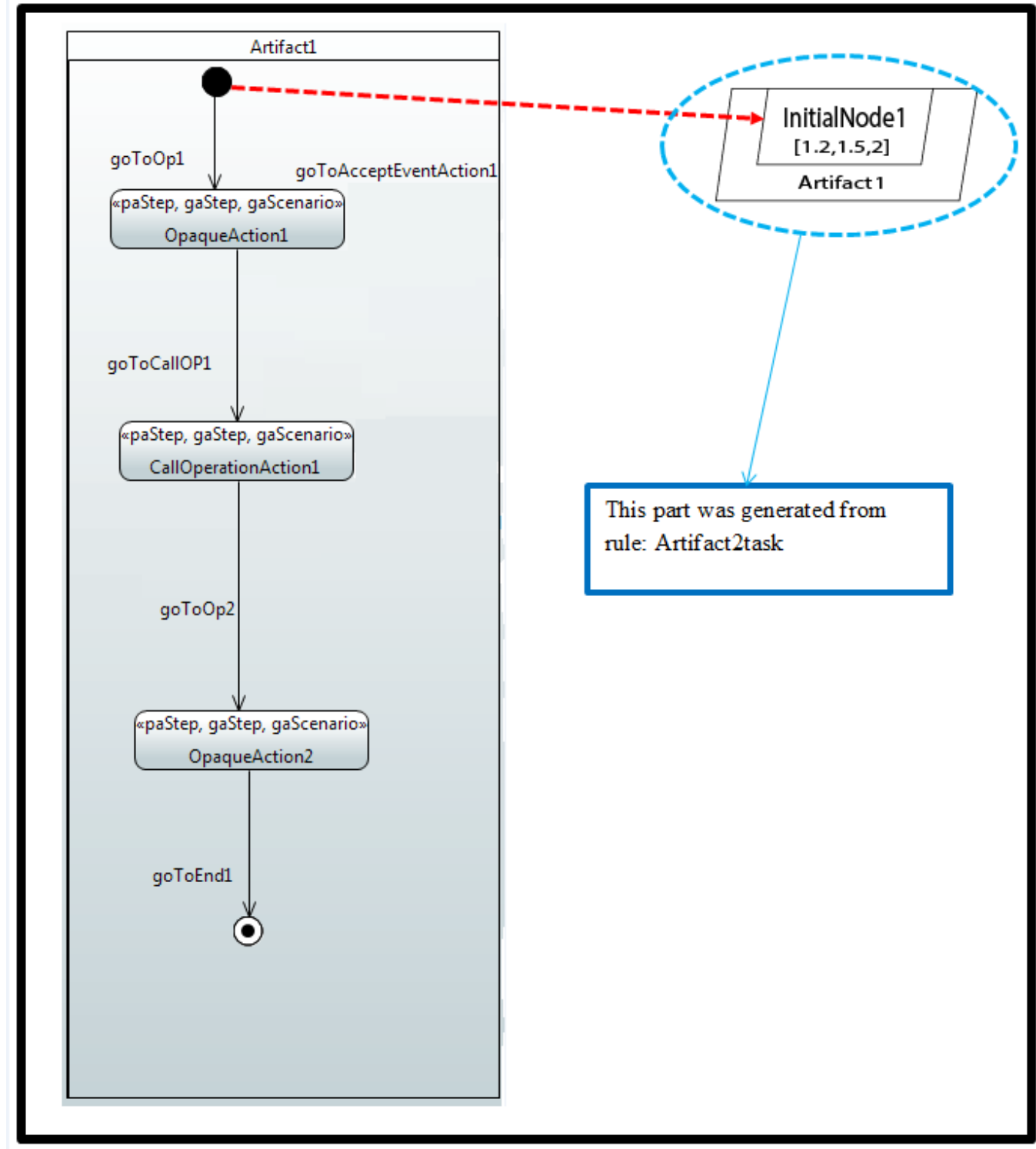

Figure 5-7. Transformation rule example - InitialNode2entryPh1Ph2

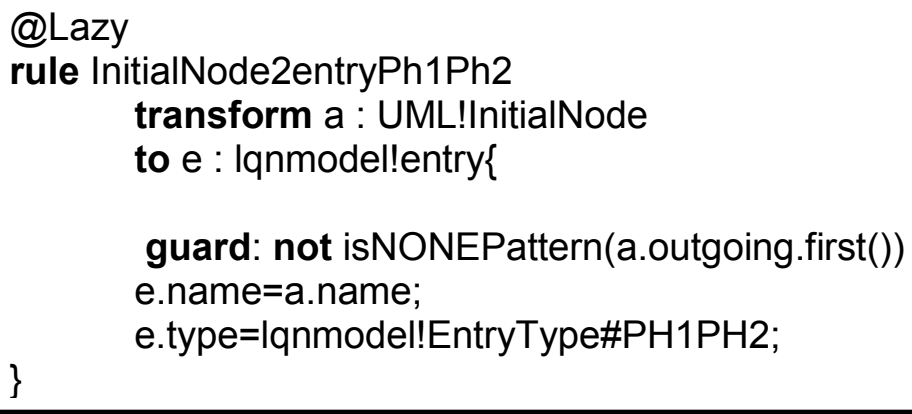

Code Fragment 5-6. Transformation rule - InitialNode2entryPh1Ph2 


\subsubsection{Rule AcceptEventAction2entryPh1Ph2}

The structure of this rule is similar to the previous one, with the difference that "AcceptEventAction" is transformed into an LQN "entry", as shown in Figure 5-8 and Code Fragment 5-7 below. Its "guard" condition is also similar to the previous rule.

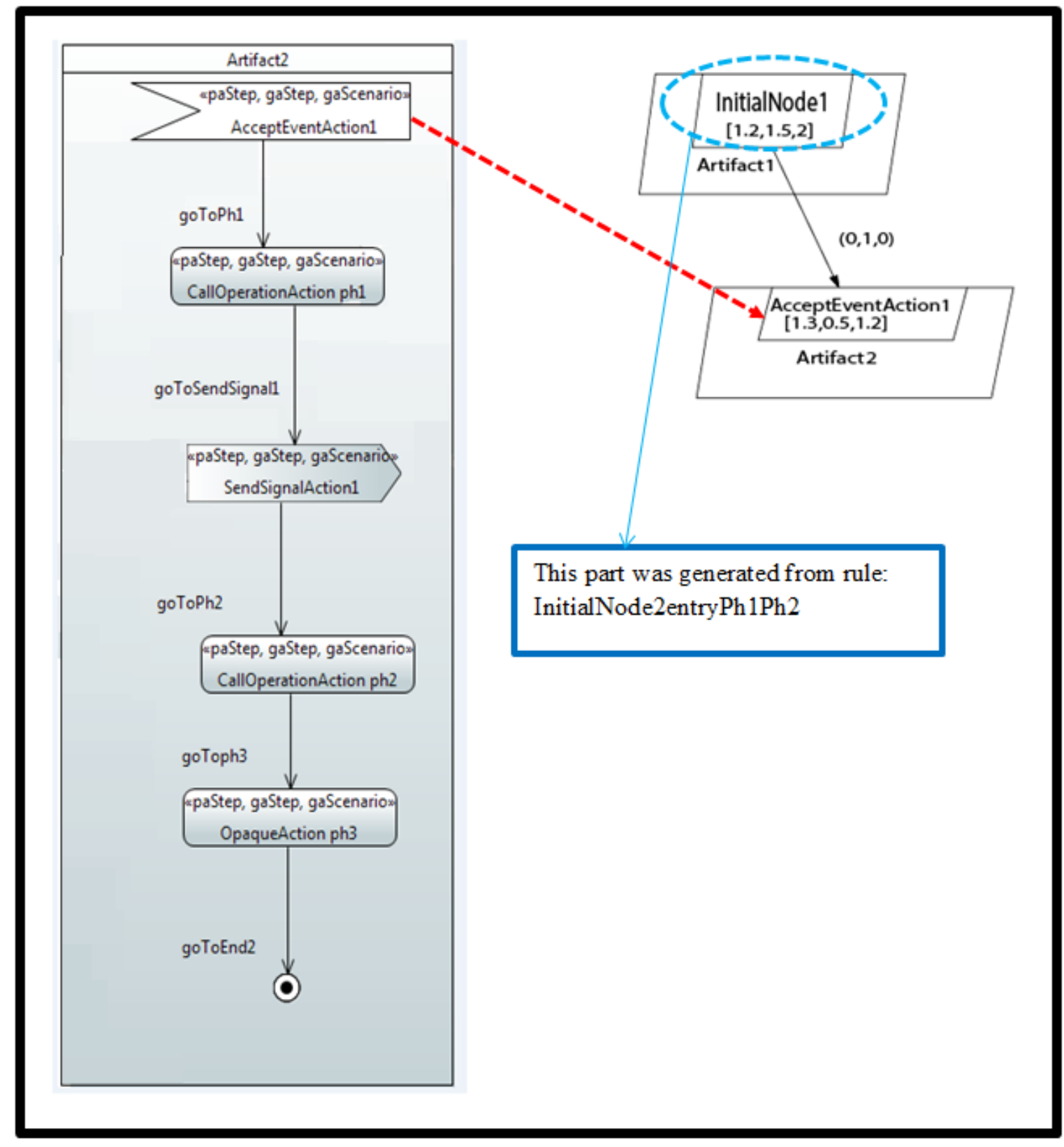

Figure 5-8. Transformation rule example - AcceptEventAction2entryPh1Ph2 


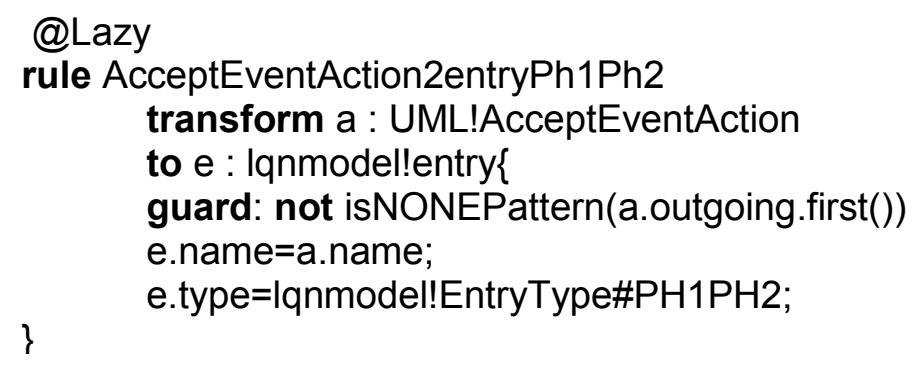

Code Fragment 5-7. Transformation rule - AcceptEventAction2entryPh1Ph2

When the negation of "isNONEPattern()" operation evaluates to "true", the guard's condition becomes satisfied. As mentioned above, this operation takes as parameter a ControlFlow outgoing from an "AcceptEventAction", starts traversing the graph upward until it finds the origin of the flow. Unlike the last rule whose origin was "InitialNode", this time the origin is "AcceptEventAction". As we can see in Figure 5-8, there are three "ActivityNode" in this flow graph excluding "AcceptEventAction", "SendSignalAction" and "ActivityFinalNode", contained in the "ActivityNode" collection. If the size of this collection is not greater than three, the pattern is "PH1PH2".

\subsubsection{Rule AcceptEventAction2entryNone}

This rule similar to the previous one, with the difference that it transforms an "AcceptEventAction" into an LQN "entry" with a "NONE" pattern, as shown in Figure 5-9 and Code Fragment 5-8.

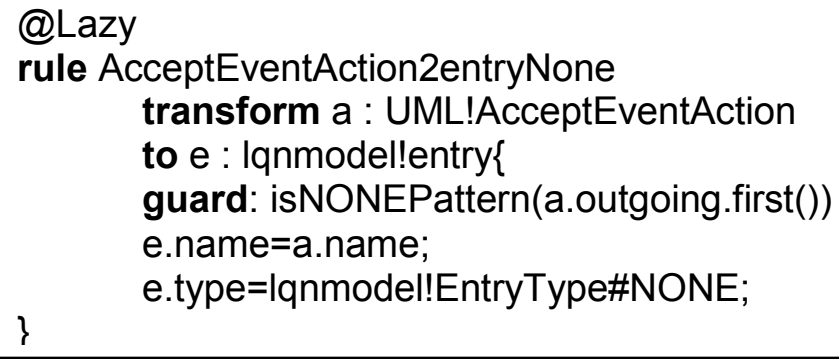

Code Fragment 5-8. Transformation rule - AcceptEventAction2entryNone 


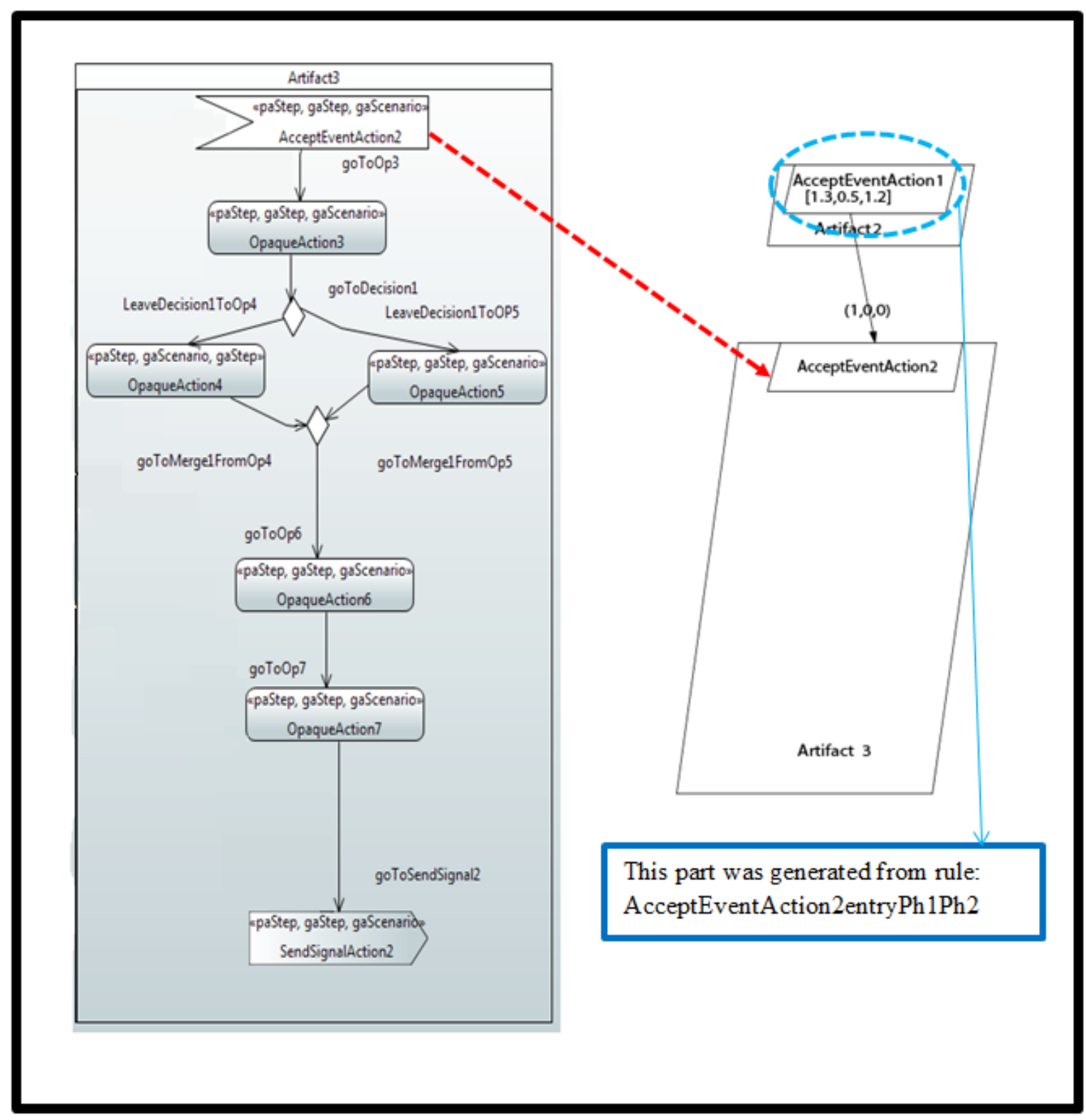

Figure 5-9. Transformation rule example - AcceptEventAction2entryNone

The guard condition of this rule includes the operation "isNONEPattern()" (without negating it). This time, after traversing the flow graph downward to the end, the extracted "ControlNode" collection contains one "DecisionNode" and one "MergeNode" as we can see in Figure 5-9, which are both subtypes of a "ControlNode". This means that this graph is a "NONE" pattern graph so that the attribute "type" of the transformed LQN "entry" becomes "NONE". 


\subsubsection{Rule OpaqueAction2Activity}

This rule maps UML "OpaqueAction" to LQN "activity" of the target model. As shown in Figure 5-10 and Code Fragment 5-9, there are some applied stereotypes on each "OpaqueAction" object. This rule looks for the "GaScenario" stereotype from the collection of stereotypes collected for each "OpaqueAction"; if its attribute "hostDemand" is initialized, it will assign the value of that to the attribute "hostdemandmean" of the LQN "Activity".

"Scenario" and "Step" stereotypes can be applied to a broad set of behaviorrelated elements, which are covered by the UML2 metaclass NamedElement, such as Actions. GaScenario" stereotype maps the elements of the "BehaviorScenario".

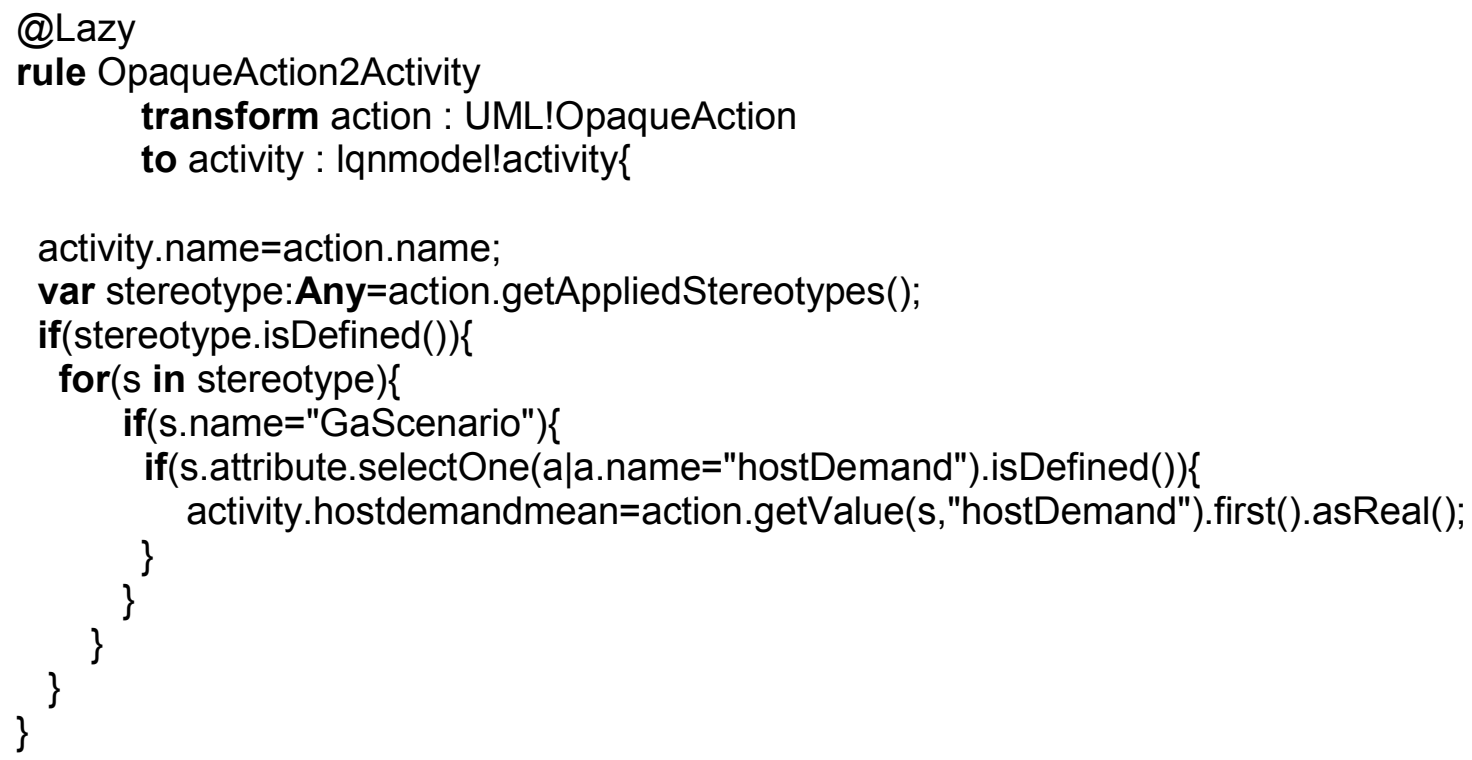

Code Fragment 5-9. Transformation rule - OpaqueAction2Activity 


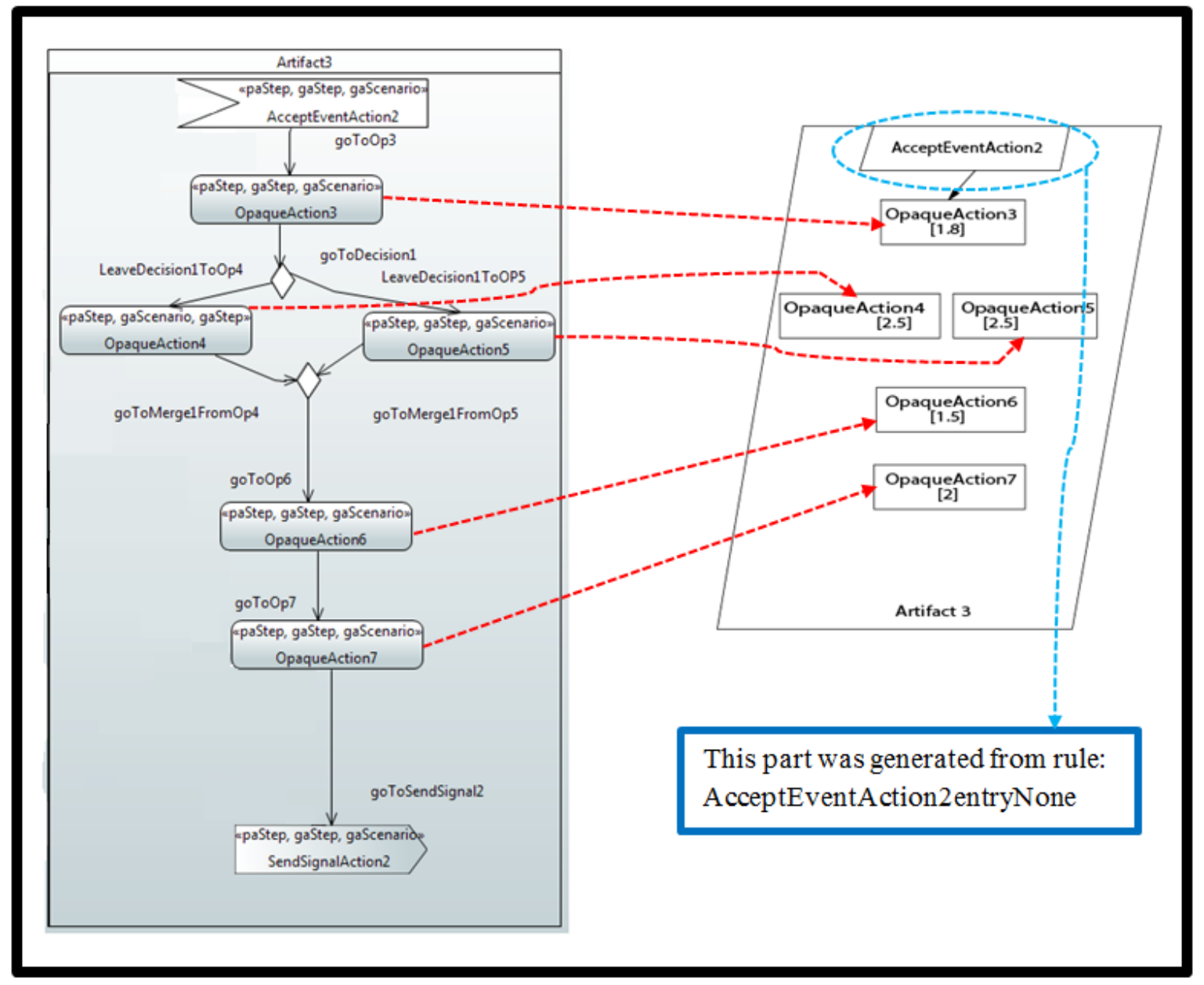

Figure 5-10. Transformation rule example - OpaqueAction2Activity

A "Scenario" takes system-level behavior, then it attaches allocations and resource usages to it. It consists of sub-operations which are called "Steps". "hostDemand" is an attribute of the "GaScenario" stereotype, which represents the CPU demand in units of time and all Steps are expected to be on the same host [MART11]. Similarly, "hostdemand-mean" is the mean service time demand for the activity in the LQN domain [LQN15]. "GaStep" stereotype represents the Step domain element, is a sup-type of the "GaScenario" stereotype. It is part of the "Scenario" and it can be depicted in sequence with other actions [MART11] as shown in Figure 5-10. Therefore, "PaStep" which represents Step in Performance Analysis Modeling (PAM) profile, is sup-type of 
"GaStep". To provide performance interpretations, "PaStep" inherits some properties of its superclass "GaStep". It should be noted that "PaStep" without a refining scenario is a basic sequential execution step on a host processor, while with a refining scenario it is a larger unit of behavior [MART11]. In performance modeling, it is just required to apply "PaStep" stereotype on each action of the behavior scenario.

\subsubsection{Rule DecisionNode2Precedence}

This rule transforms UML "DecisionNode" to LQN "precedence". As depicted in Code Fragment 5-10 and Figure 5-11. According to Code Fragment 5-10, the two LQN "pre" and "postor" elements are instantiated to be added as children of the "precedence",

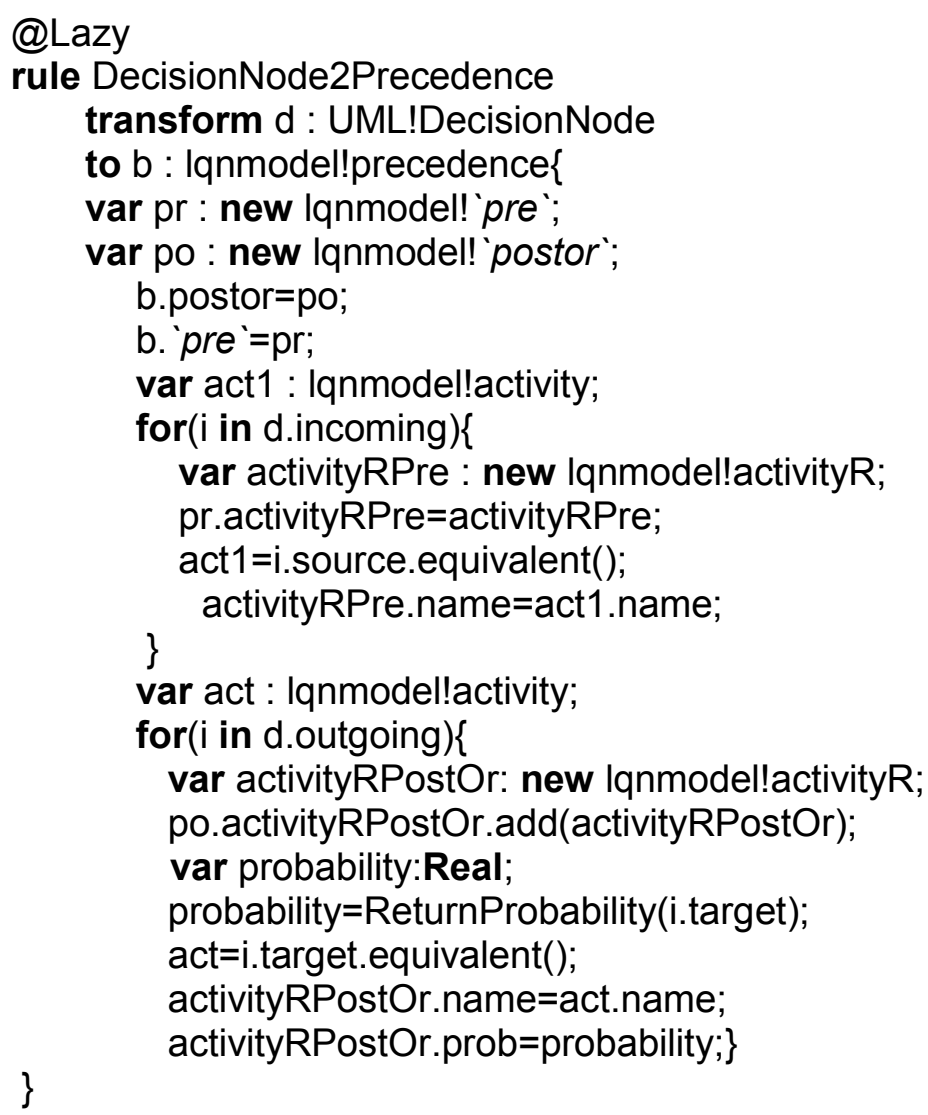

Code Fragment 5-10. Transformation rule - DecisionNode2Precedence 


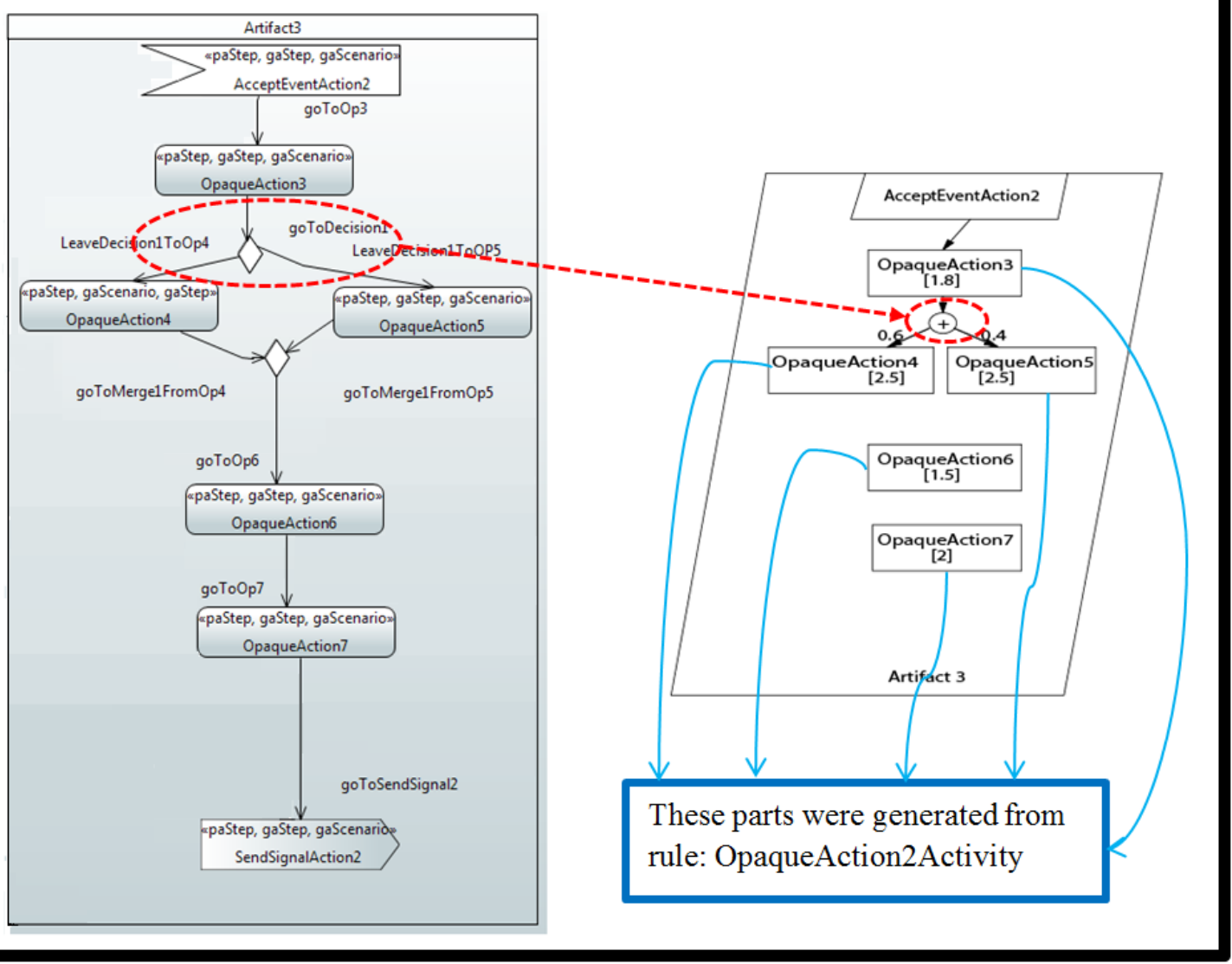

Figure 5-11. Transformation rule example - DecisionNode2Precedence

as defined in the LQN metamodel. For each "ControlFlow" item in the "incoming" collection of the "DecisionNode", one LQN "activityR" element is instantiated to be assigned as an "activity" role name of the LQN "pre" instance. LQN "activityR" represents an LQN "activity" which comes before the "precedence" in the target model as shown in Figure 5-11. The attribute "name" of LQN "activityR" is the same as the "name" of its mirrored LQN "activity", which is transformed by invocation of ETL buitin "equivalent()" method on the "source" of each "ControlFlow" object of the "incoming" collection. In addition, the same procedure will be repeated, but this time for each "ControlFlow" element in the in the "outgoing" collection of the "DecisionNode". LQN "activityR" is instantiated again to be added as an "activity" of the LQN "postor" 
instance. The multiplicity of the "activityR" instances that could be added to the "postor" object is more than one, that's why the "add()" operation is used. However, the multiplicity of "activityR" of the "pre" is just one, so a simple assignment statement does the job. After getting the mirrored "activity" of the "activityR" by calling "equivalen()" method on the "target" of the "ControlFlow" of the "outgoing" collection, its "name" and "prob" attribute values will be assigned to the respective attributes of the "activityR"

\subsubsection{Rule MergeNode2Precedence}

This rule maps UML "MergeNode" to LQN "precedence", as shown in Code

Fragment 5-11 and Figure 5-12. The structure of this rule is as the previous one.

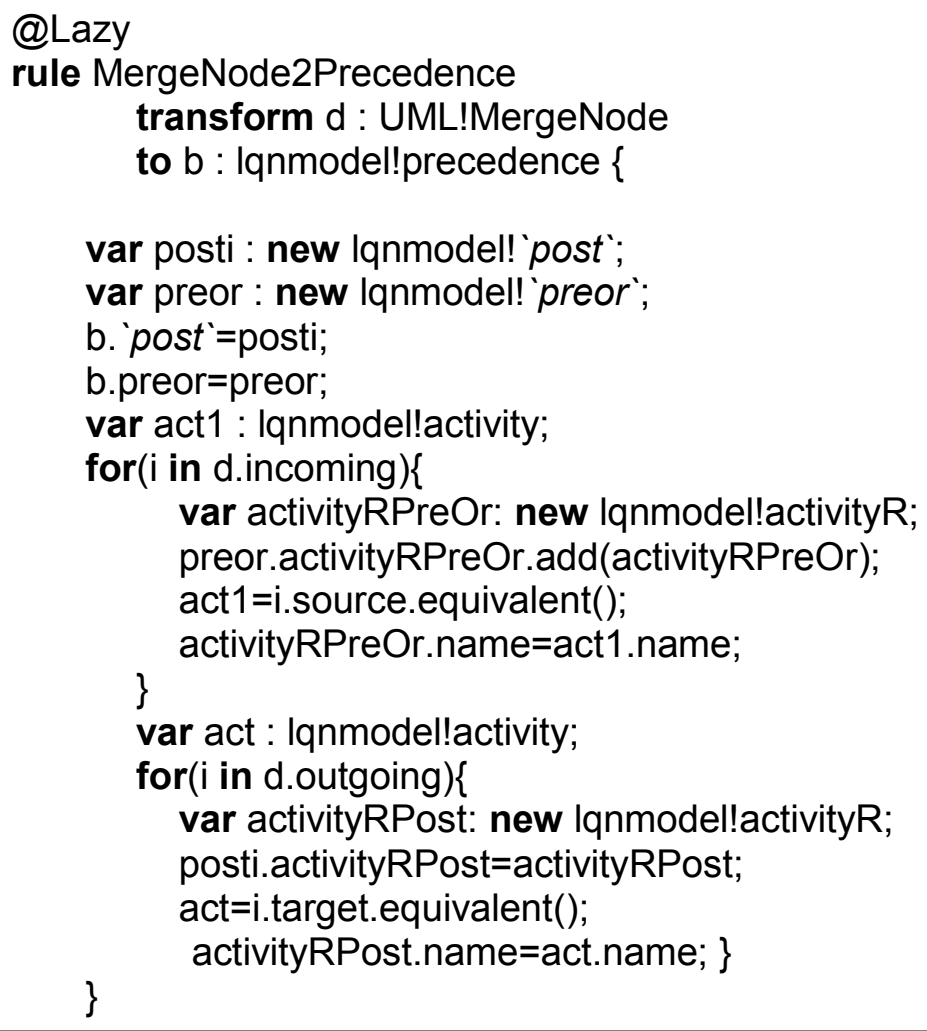

Code Fragment 5-11. Transformation rule - MergeNode2Precedence 


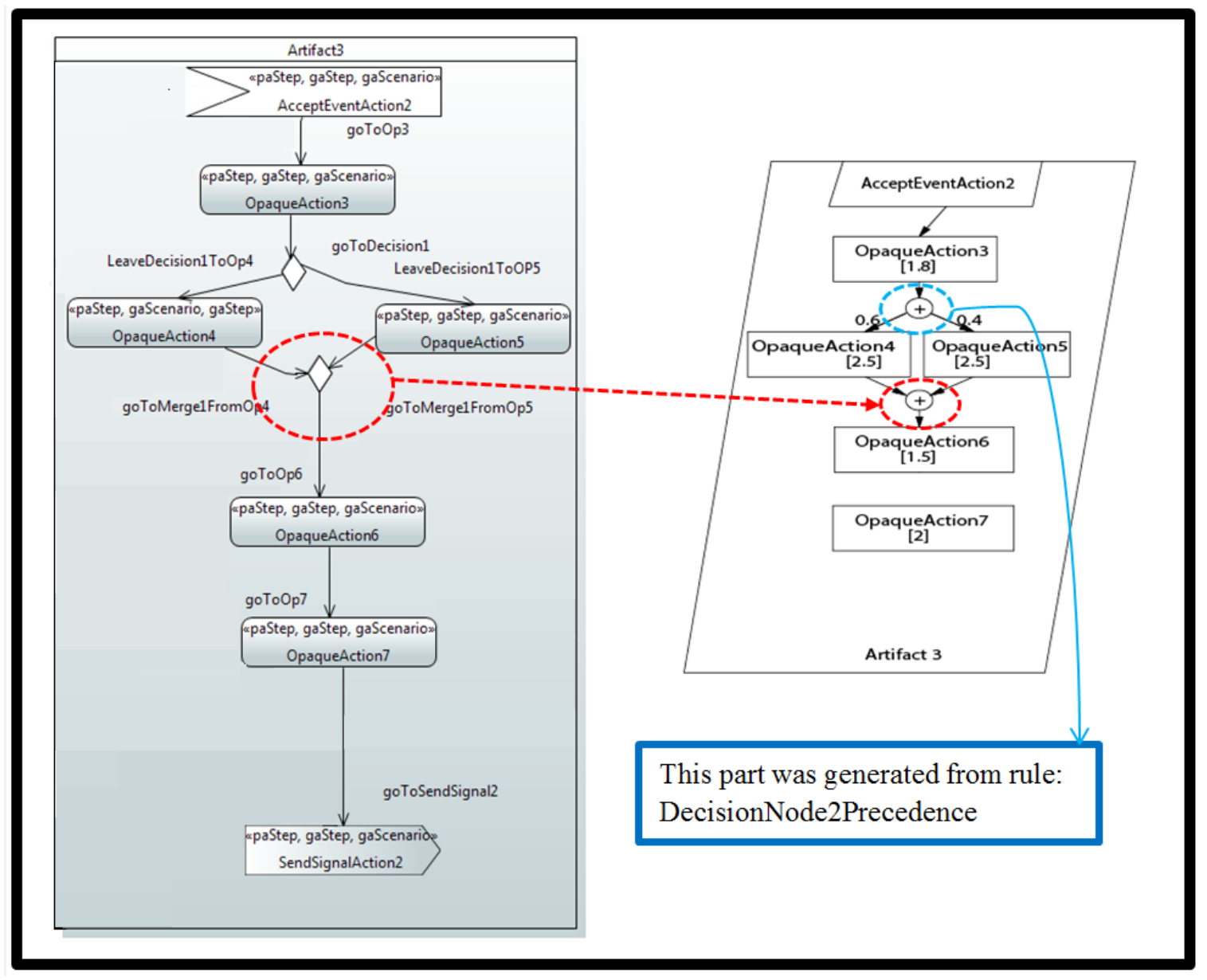

Figure 5-12. Transformation rule example - MergeNode2Precedence

The differences are the following: the LQN "post" and ""preor" components are instantiated to be added as children of the "precedence". Like before, for each element of the "incoming" and "outgoing" collections, LQN "activityR" is instantiated; it is important that the multiplicity of the "activityR" instances added to the "preor" object is more than one, while the multiplicity of the "activityR" instances assigned to the "post" object is just one.

\subsubsection{Rule ControlFlow2Precedence}

This rule transforms UML "ControlFlow" to LQN "precedence" as we can see from the Code Fragment 5-12 and Figure 5-13. The structure of this rule is also as similar 


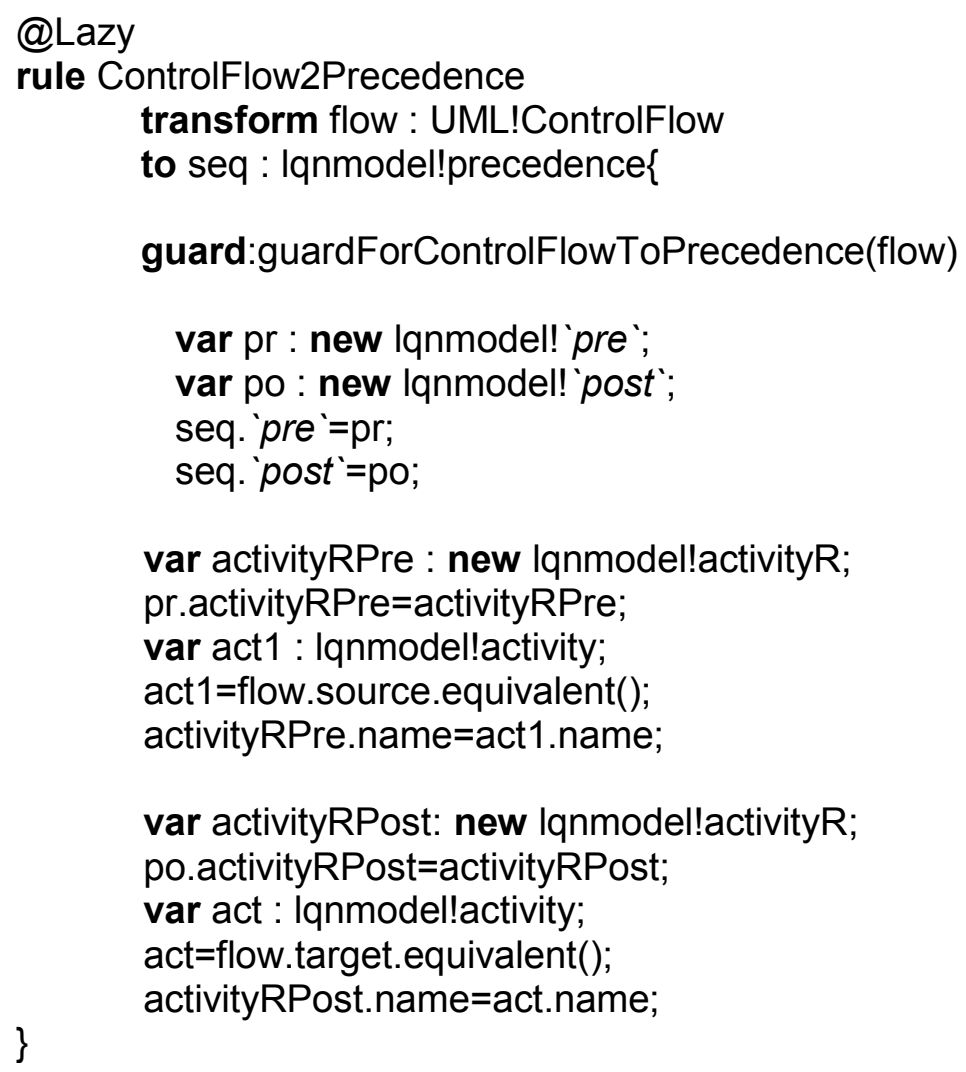

\section{Code Fragment 5-12. Transformation rule - ControlFlow2Precedence}

to the last two rules, 5.1.11 and 5.1.10. The major difference is having a "guard" condition. The other difference is that the instantiated components, which are added as children of "precedence" are of LQN "pre" and "post" types. Like the last two rules, "activityR" instances are instantiated to be assigned as an "activity" of the LQN "pre" and "post" objects. It should be noted that the multiplicity of the "activityR" instances assigned to both LQN "pre" and "post" is just one for each, so simple assignments are enough. As already mentioned, this transformation will be run if its guard condition, "guardForControlFlowToPrecedence()" is satisfied. This operation takes UML "ControlFlow" as an argument and checks some conditions on it. If the operation evaluates to "true", the targeted "ControlFlow" will be transformed to LQN "precedence". First, the operation checks whether the "inPartition" property of the 
"ControlFlow" is defined or not, meaning that it is not a border-crossing "ControlFlow". Then it checks to find out if the "ControlFlow" is part of a flow graph of type "NONE". Finally, it should not be an incoming or outgoing edge to some "ControlNode" such as "DecisionNode" and "MergeNode". If such is the case, the guard's condition becomes "true" and the transformation runs.

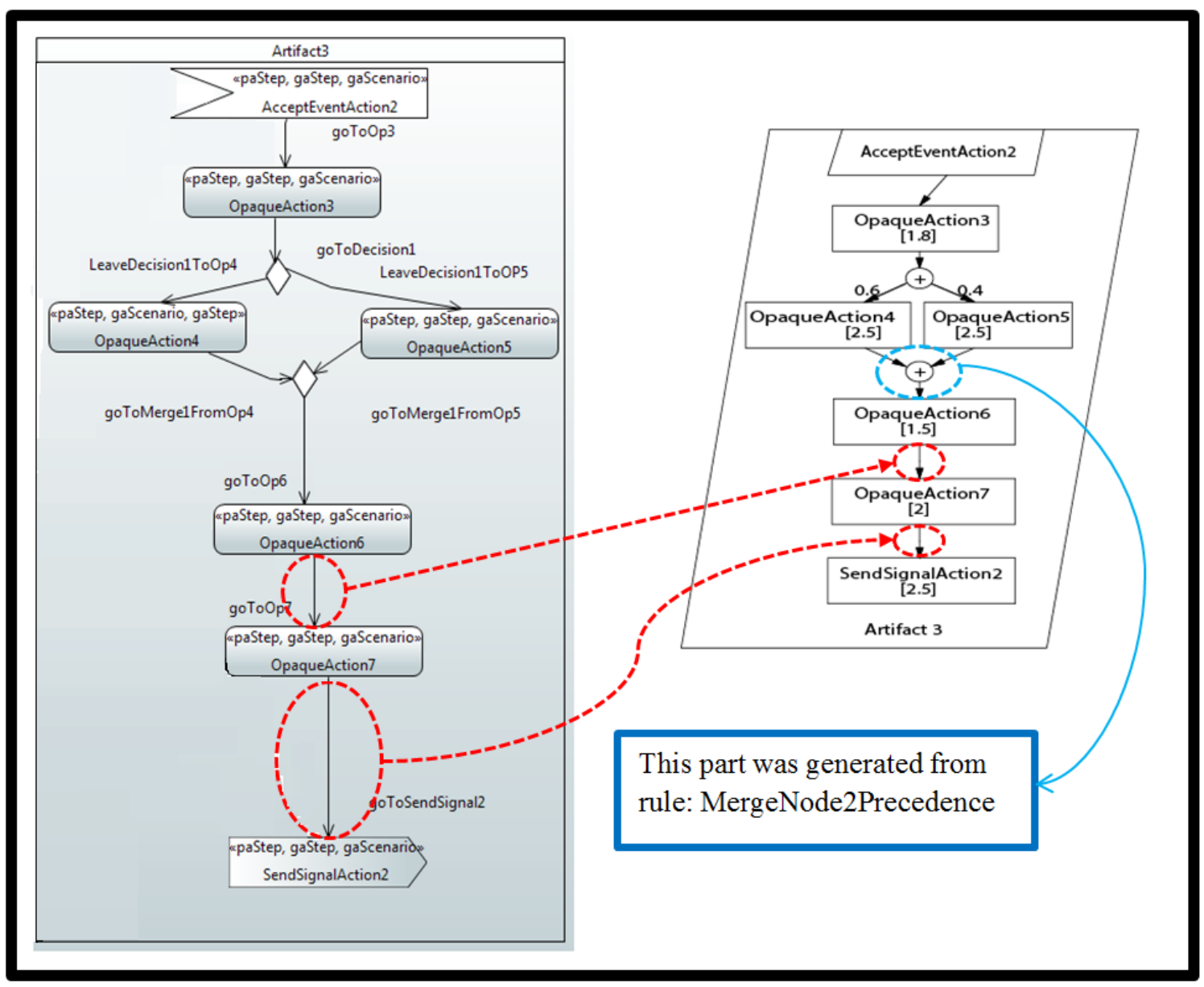

Figure 5-13. Transformation rule example - ControlFlow2Precedence

\subsubsection{Rule SendSignalAction2Activity}

This rule transforms UML "SendSignalAction" to LQN "activity" of the target model as depicted in Figure 5-14 and Code Fragment 5-13. The structure of this rule is similar to the rule 5.1.9, "OpaqueAction2Activity". The only difference is its guard 
condition. Operation "guardForSendSignalAction2Activity()"defined as a guard for this rule, gets an "incoming" element typed "ControlFlow" to the "SendSignalAction". There are some conditions that "ControlFlow" needs to meet in order for the guard operation to be satisfied: a) the "ControlFlow" should not be border-crossing, meaning that it is located inside an "ActivityPartition" and its "inPartition" attribute has to be defined; and b) this "ControlFlow" and its "SendSignalAction" target are parts of a "NONE"-type flow graph. If these conditions are met, the mentioned "SendSignalAction" will be mapped to its corresponding LQN "activity".

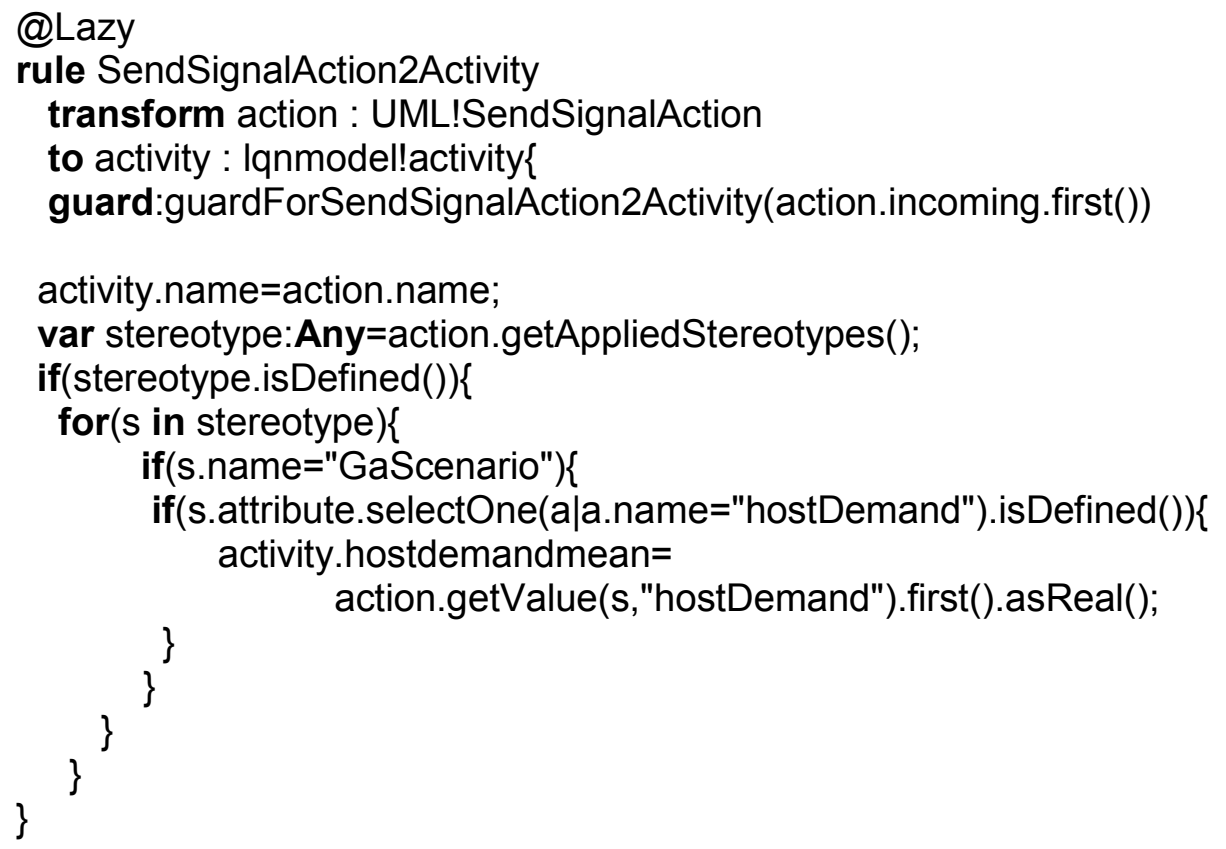

Code Fragment 5-13. Transformation rule - SendSignalAction2Activity 


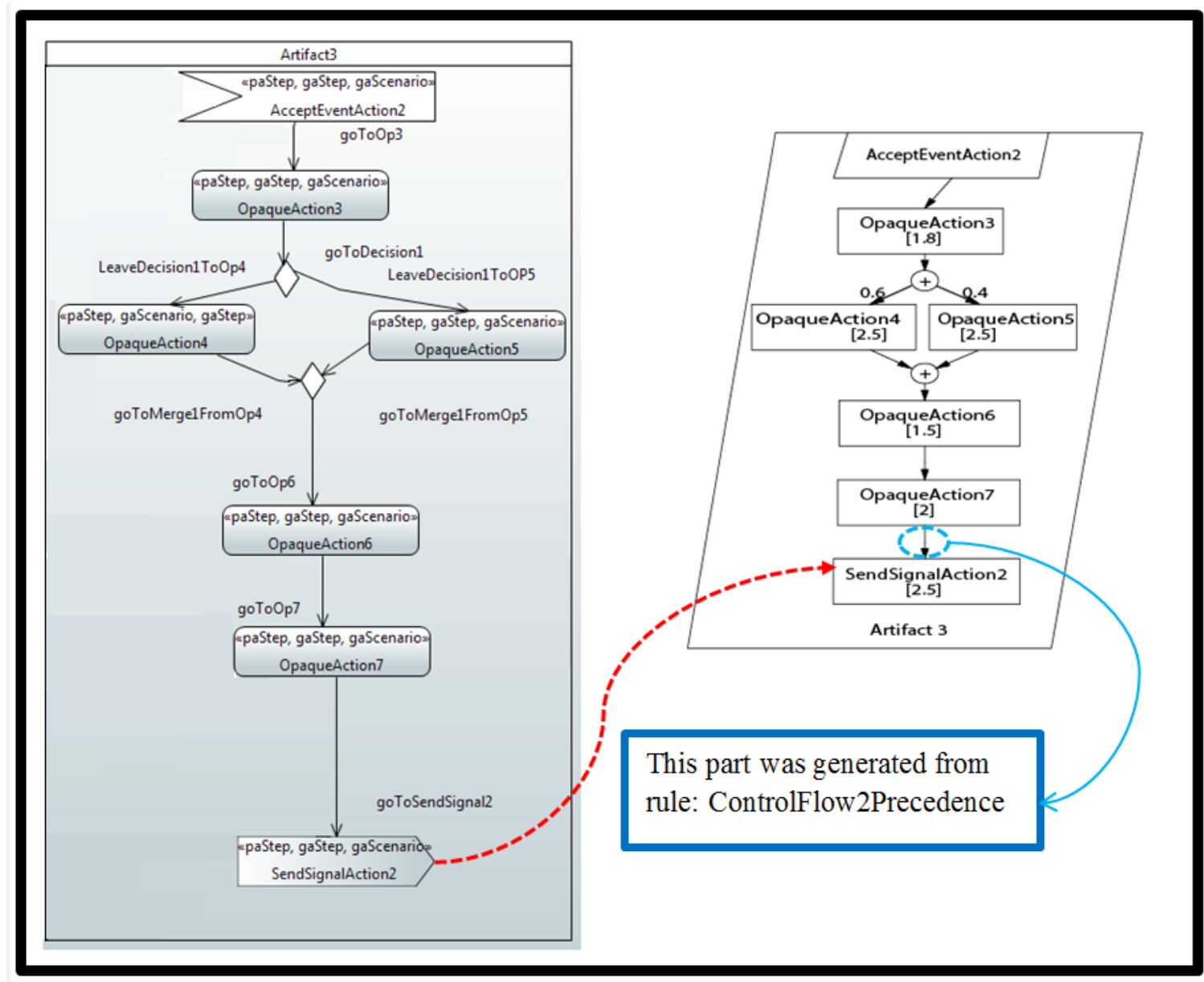

Figure 5-14. Transformation rule example - SendSignalAction2Activity

\subsubsection{Rule CallOperationAction2Activity}

This rule transforms UML "CallOperationAction" to LQN "activity" of the target model as we can see in Code Fragment 5-14 and Figure 5-15. The structure of this rule is similar to the previous rules 5.1.13 and 5.1.9. Like the other two rules; first, it collects the collection of stereotypes applied on the "CallOperationAction"; then if it finds a "GaScenario" stereotype, it will assign the value of stereotype's "hostDemand" attribute to the "host-demand-mean" property of the LQN "activity". Besides, it also looks for the "GaStep" stereotype; if its attributes "rep" and "prob" are defined, it will take their values and multiply them to find a new value which will be used shortly. The attribute "prob" 


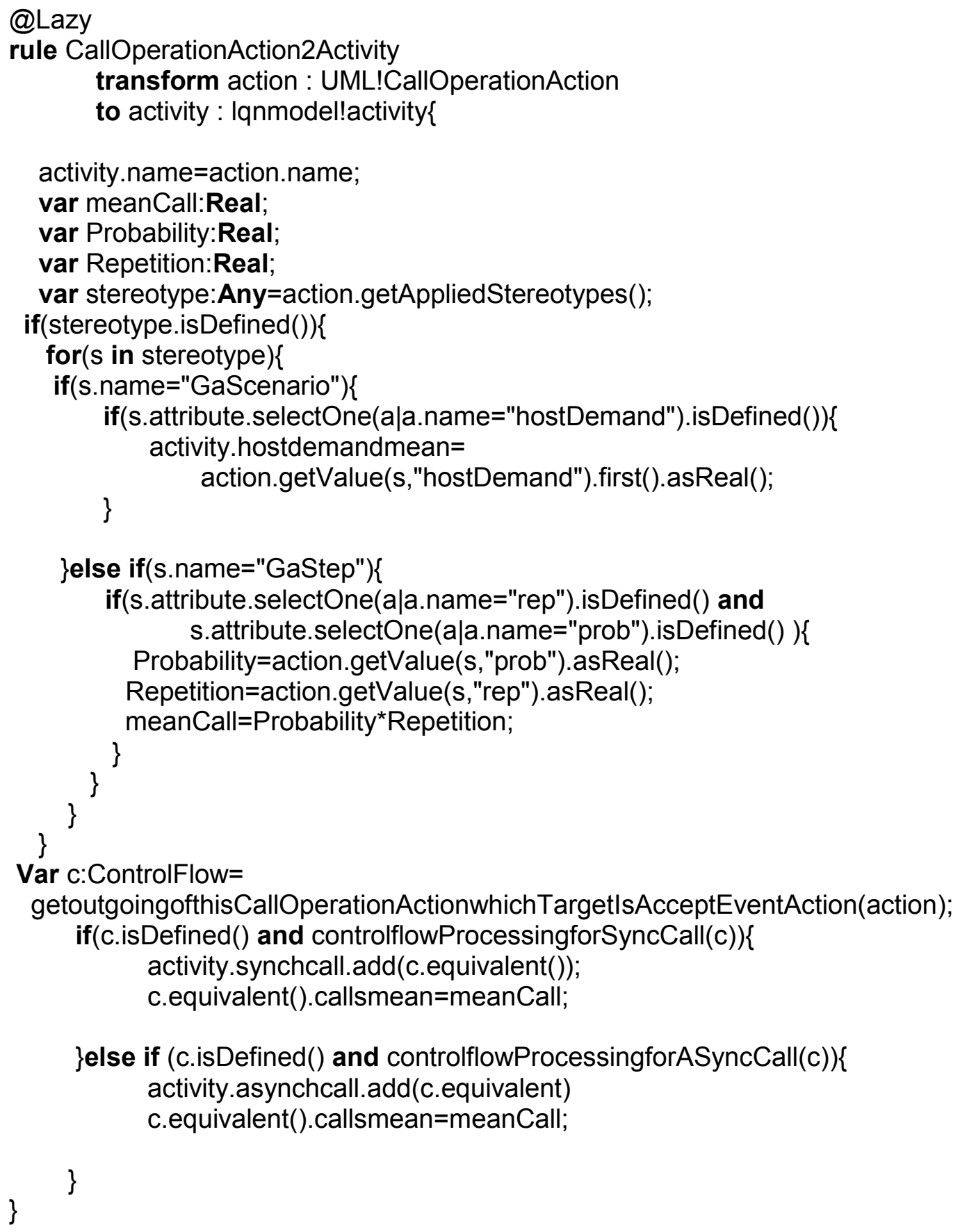

Code Fragment 5-14. Transformation rule - CallOperationAction2Activity 


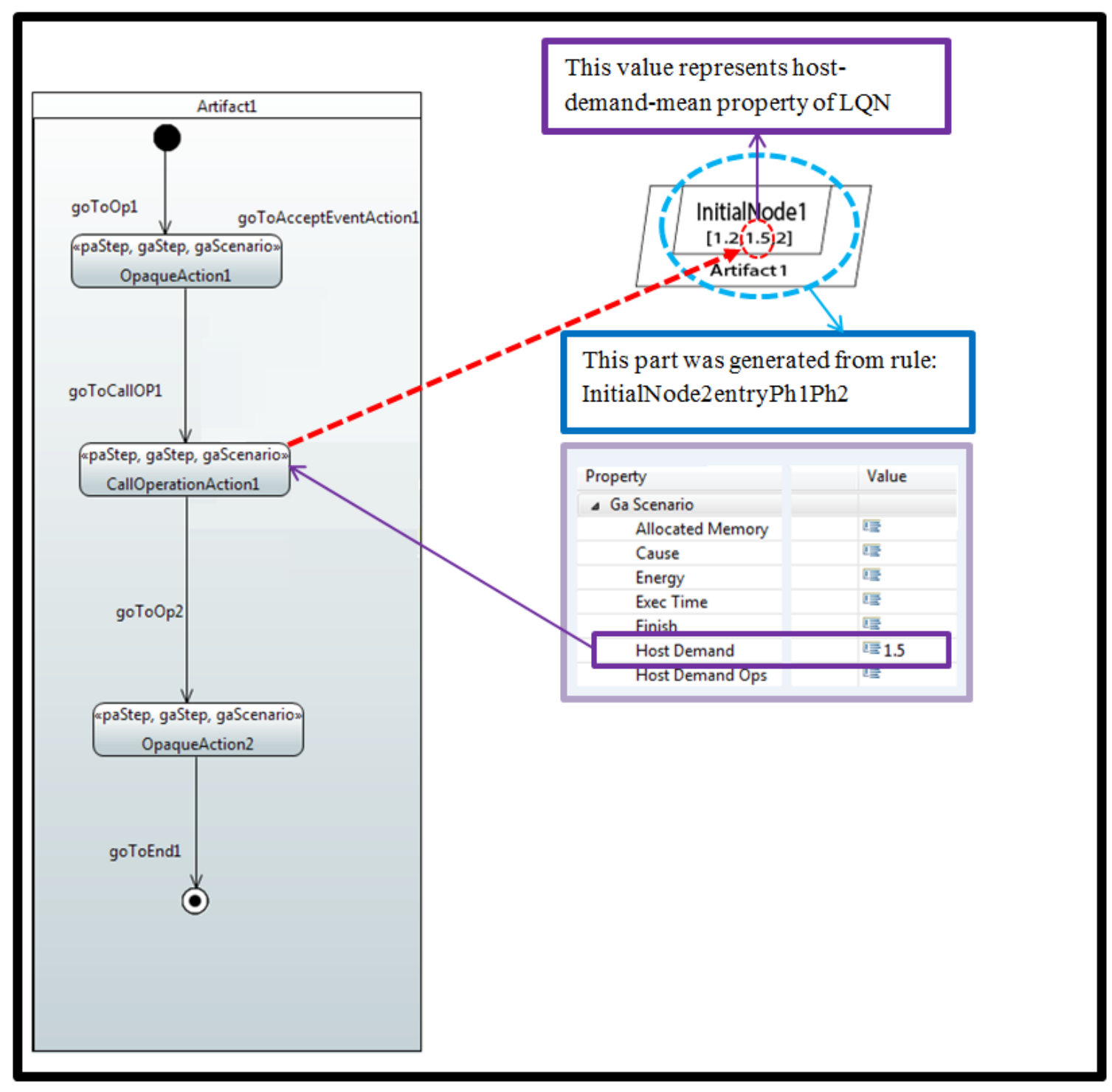

Figure 5-15. Transformation rule example - CallOperationAction2Activity

represents the probability of the step to be executed for a conditional execution and "rep" represents the actual or average number of repetitions of an operation or loop on the UML domain [MAR11]. By multiplying these values we obtain a new value which will be assigned to the "calls-mean" attribute of the LQN "synch-call" or "asynch-call". The attribute "calls-mean" defines the mean number of requests from activities to entries in the LQN domain [LQN15]. The "outgoing" of the "CallOperationAction", which is of type border-crossing "ControlFlow" and is also "incoming" to the "AcceptEventAction" 
located in another "ActivityPartition", represents a call request in the UML domain. If this call request exists and its type is "Synchronous" (meaning that there will be a send reply back to the "CallOperationAction"), its corresponding LQN "synch-call" will be added to the LQN "activity"; otherwise, if its type is "Asynchronous" meaning that there is no reply sent back, its respective LQN "asynch-call" will be added to the LQN "activity".

\subsubsection{Rule ControlFlow2synchcall}

As we can see from Code Fragment 5-15 and Figure 5-16, this rule maps a UML "ControlFlow" to LQN "synchcall". There is a guard condition which needs to be met before the transformation executes. Operation "controlflowProcessingforSynchCall()" should evaluate to "true" in order to satisfy the guard condition. This operation takes "ControlFlow" as an argument and checks whether this "ControlFlow" meets the condition of "Synchronous" call; if so, the targeted "ControlFlow" will be transformed and the name of its "target" property will be given to the "dest" attribute of its corresponding LQN "synch-call". The "dest" attribute defines the name of the "entry" which the call requests are sent to [LQN15]. This operation looks for the "outgoing" property of the "AcceptEventAction" which is the "target" property of the passed "ControlFlow" argument. The flow graph will be traversed recursively until "SendSignalAction" is reached. If the "target" property of the "outgoing" property of this "SendSignalAction" is equal to the "source" property of the passed "ControlFlow" argument, this operation evaluates to "true",meaning that it represents a "synch-call". 


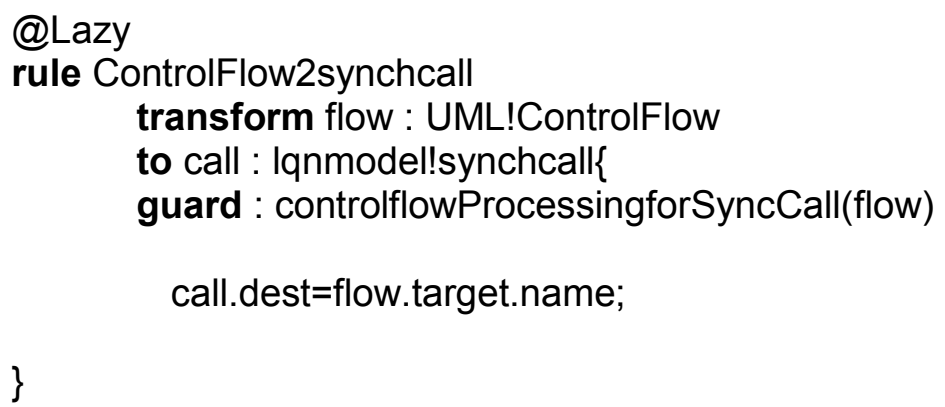

Code Fragment 5-15. Transformation rule - ControlFlow2synchcall

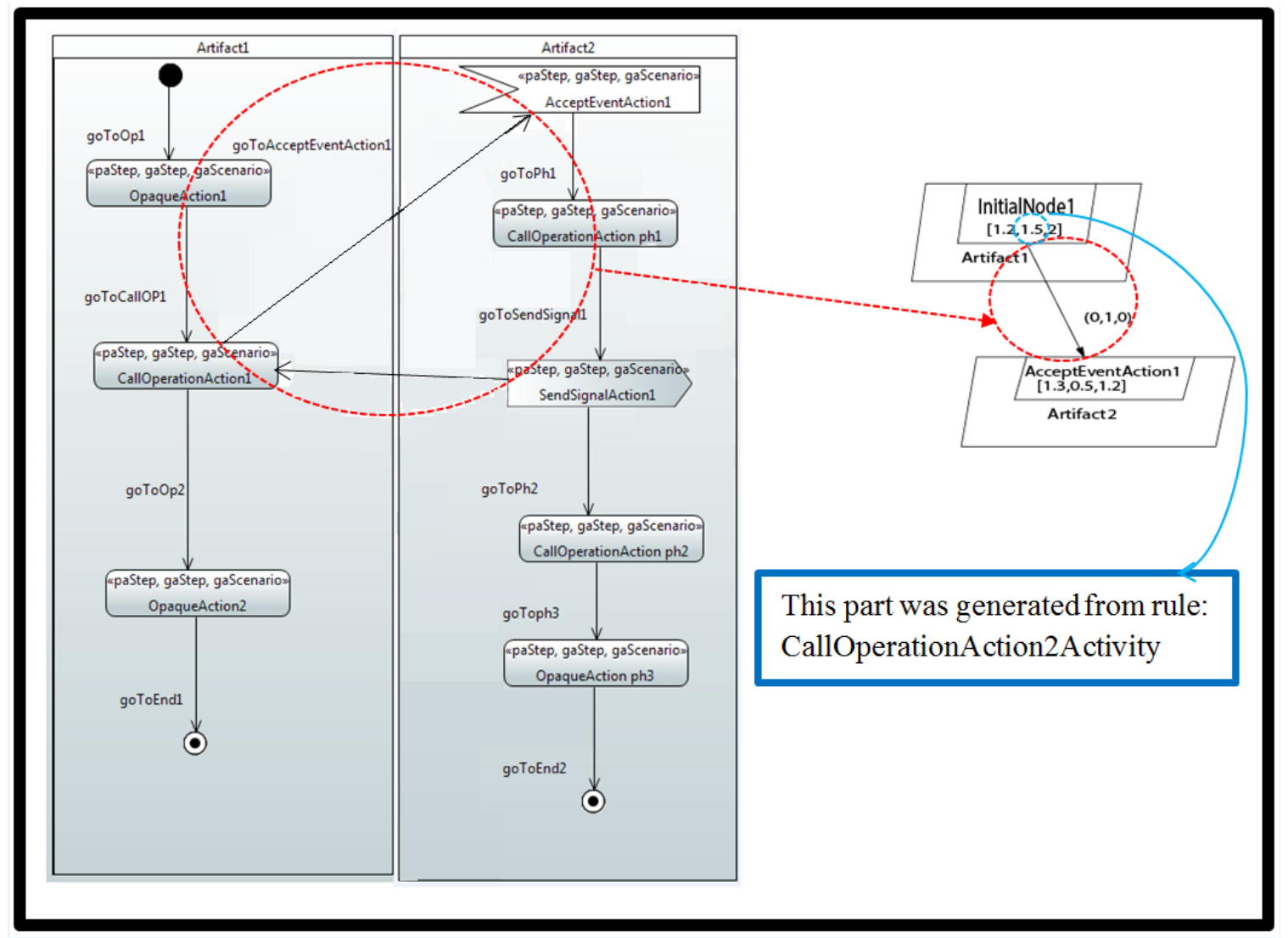

Figure 5-16. Transformation rule example - ControlFlow2synchcall

\subsection{Pre-transformation processing}

As already mentioned above, some properties of the UML source model need to be assigned before running the transformation. For example, as shown in Figure 5-6, "inPartition" property of a non-border crossing "ControlFlow" has to be set manually. In 
order to reduce the effort of the modeler and eliminate error-prone manual operations such as this, an Epsilon EOL script can be written. This program reads the UML source model given to it as an input and writes back to this model assigning values to properties. Code Fragment 5-16 shows an example of an EOL script that collects all the "ControlFlows" which the "inPartition" properties of their "source" and "target" are the same. Then it assigns the value of the "inPartition" property of "source" of the "ControlFlow" to the "inPartition" property of the "ControlFlow".

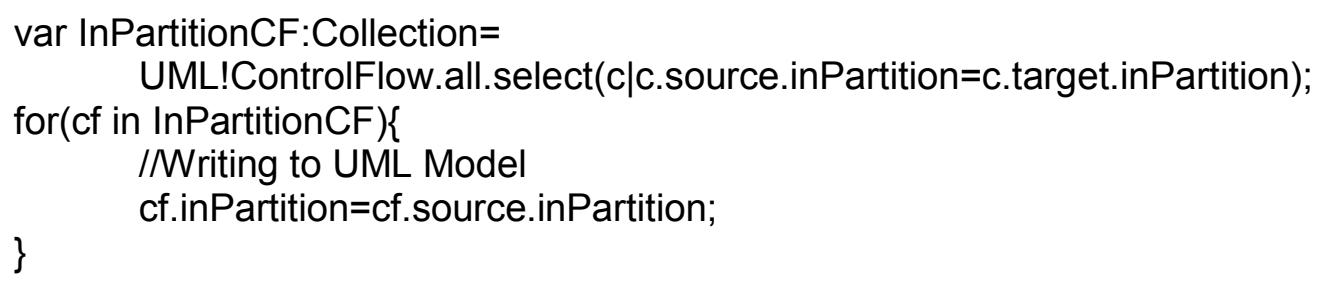

Code Fragment 5-16. Example of EOL Script writing to the UML Source Model

Other preliminary verifications can be done in this way, as for example checking whether the necessary attributes of MARTE stereotypes are set. If not, default values can be assigned and the user alerted before running the transformation.

\subsection{Post-transformation processing}

The result of the transformation is a preliminary LQN model in XML format, as explained in Section 4.1. (See an example of such file in Appendix B1 for the LQN model generated for the running UML case study given in Figure 3-1 and 3-2). This model is meant to be an input to the LQNS solver, so it needs to be reformatted in order to be compatible with the solver. Most of the changes are applied to this XML file automatically using a JAVA program. This program reads the preliminary XML file, looks for some tag values (as explained in Section 4.1) and replaces them with the correct 
ones. It also adds a solver-params element (that cannot be generated from the UML model), which will be used by the solver. However there is one change that has to be done manually in the element starting with the lqn-model tag value, where the address of the XML schema and the path of the LQNS solver on the local host have to be set. Appendix B.2 shows the outcome of the post-processing step for the preliminary file from Appendix B.2. The solver-params element is shown in italics, and the parts that are changed manually are underlined.

\subsection{Extracting LQN results from LQN output files}

By writing an EOL script which takes an LQN output generated by the LQNS solver in a XML format, LQN performance attributes can be extracted from the output file generated by the LQNS solver. Code Fragment 5-17 shows an example which takes an output model given in Appendix B.3 as an input and Figure 5-17 displays the results on the Epsilon console.

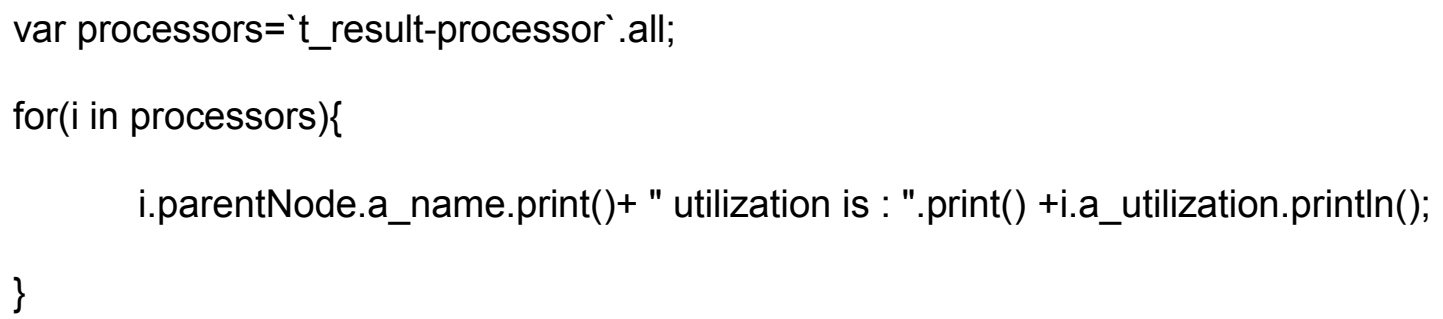

Code Fragment 5-17. Example of EOL Script reading from LQN Target Model

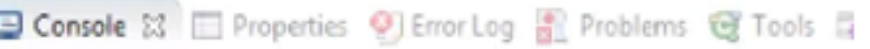
Epsilon

Devicel utilization is : 0.278404

Device2 utilization is : 0.177705

Device3 ut1lization 15 : 0.610119

Figure 5-17. Example of Epsilon Console displaying the results 


\section{Chapter: Transformation Evaluation}

This chapter presents several test cases which were used for verification and validation of the developed transformation process. We also apply our technique to an E-commerce case study published by other authors in [Cort11], in order to mitigate the possible bias when the author of a software product builds the test cases for verification.

\subsection{Test cases}

According to the IEEE standard IEEE-STD-610 which identifies terms currently in use in the computer and software field, the definitions for software verification and validation are as follows:

- Software Verification: The process of evaluating software to determine whether the products of a given development phase satisfy the conditions imposed at the start of that phase.

- Software Validation: The process of evaluating software during or at the end of the development process to determine whether it satisfies specified requirements.

The verification of the transformation developed in the thesis was done by testing it with different test cases. For testing purposes, twelve transformation features were identified and ten test cases were used to verify whether the transformation produced the expected output. Eight out of the test case are simple models, focused on certain features (T1 to T8), as explained below. The ninth test case is the running case study CS1 (see Figure 3-1 and 3-2 for the UML model, Appendix B for the LQN model in XML format and Figure 3.17 for the LQN graphical notation). The last test case is an example published by other authors [Cort11] shown in Figures 6-1 to 6-5. 
Table 6-1. Test Cases used for verifying different features

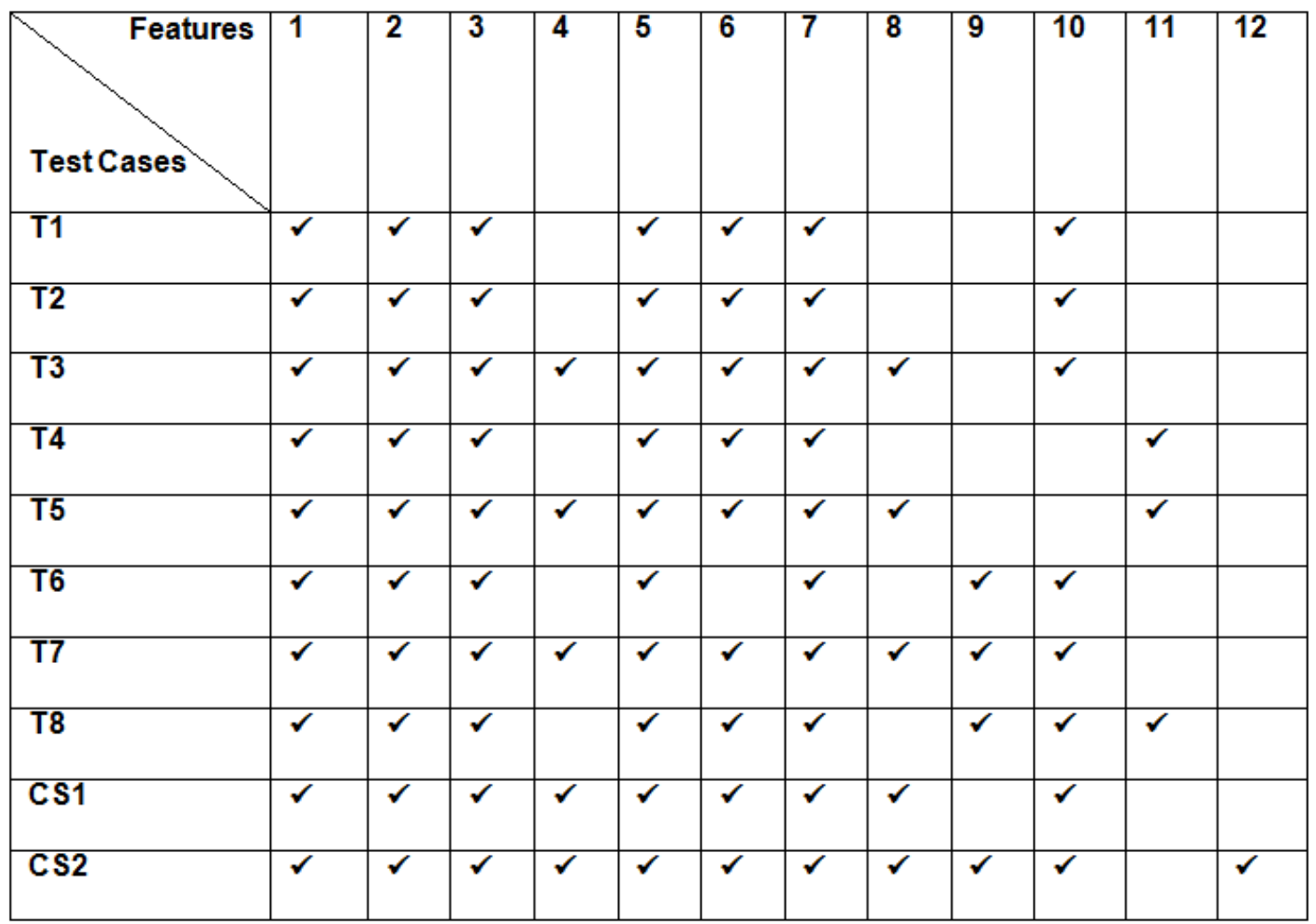

The features identified for testing are listed below, and the way in which they are covered by different test cases is shown in Table 6-1.

1. Generation of processors

2. Generation of tasks

3. Generation of entries (types PH1PH2 and NONE)

4. Generation of activity graph (pattern NONE)

5. Transformation of InitialNode

6. Handling of ActivityFinalNode

7. Transformation of ActivityNodes stereotyped with $<<$ PaStep $>>$

8. Transformation of Join, Fork, Merge and Decision nodes

9. Handling tasks with multiple entries 


\section{Handling synchronous requests}

11. Handling asynchronous requests

12. Handling multiple scenarios (each generates a different reference task)

The simple test cases $\mathrm{T} 1$ to $\mathrm{T} 8$ were designed to cover the features indicated in table 6-1 as follows:

T1: contains two processors with an artifact deployed on each one of them. The activity diagram contains two partitions interacting with each other using a synchronous call. The first partition contains a sequence of three activities starting with an InitialNode and sends a call request to the second partition, to represent a $\mathrm{PH} 1 \mathrm{PH} 2$ entry pattern, and the second partition a sequence of activities starting with an AcceptEventAction to represent a PH1PH2 entry pattern. Sending the reply back from this partition to the sender of the call after its activity phase one is the end of this sequence.

T2: contains two processors with an artifact deployed on each one of them. The activity diagram contains two partitions interacting with each other using a synchronous call. The first partition contains a sequence of three activities starting with an InitialNode and sends a call request to the second partition, to represent a $\mathrm{PH} 1 \mathrm{PH} 2$ entry pattern, and the second partition a sequence of activities starting with an AcceptEventAction to represent a PH1PH2 entry pattern. Reply is sent back from this partition to the sender of the call after its phase one, then the phases two and three are defined in sequence. This flow ends with an ActivityFinalNode.

T3: contains two processors with an artifact deployed on each one of them. The activity diagram contains two partitions interacting with each other using a synchronous call. The first partition contains a sequence of three activities starting with an InitialNode and 
sends a call request to the second partition, to represent a $\mathrm{PH} 1 \mathrm{PH} 2$ entry pattern. However, the second partition contains an activity graph starting with an AcceptEventAction to represent a NONE entry pattern. The graph contains control nodes of type decision and merge. Sending the reply back from this partition to the sender of the call after the last activity of the activity graph is the end of this graph.

T4: contains two processors with an artifact deployed on each one of them. The activity diagram contains two partitions interacting with each other using an asynchronous call. The first partition contains a sequence of three activities starting with an InitialNode and sends a call request to the second partition, to represent a PH1PH2 entry pattern, and the second partition contains a sequence of activities starting with an AcceptEventAction to represent a PH1PH2 entry pattern. There is no need to have a reply back from this partition to the sender of the asynchronous call after the first activity of the sequence. This sequence ends with an ActivityFinalNode.

T5: contains two processors with an artifact deployed on each one of them. The activity diagram contains two partitions interacting with each other using an asynchronous call. The first partition contains a sequence of three activities starting with an InitialNode and sends a call request to the second partition, to represent a $\mathrm{PH} 1 \mathrm{PH} 2$ entry pattern. However, the second partition contains an activity graph starting with an AcceptEventAction to represent a NONE entry pattern. The graph contains control nodes of type fork and join. There is no need to have a reply back from this partition to the sender of the asynchronous call after the last activity of the activity graph. This activity graph ends with an ActivityFinalNode. 
T6: contains two processors with an artifact deployed on each one of them. The activity diagram contains two partitions interacting with each other using synchronous calls. The first partition contains a sequence of three activities starting with an InitialNode and sends two call requests to the second partition, to represent a PH1PH2 entry pattern. Also, the second partition contains two sequences of activities each starting with an AcceptEventAction to represent a PH1PH2 entry pattern. Reply messages are sent back from each sequence of this partition to the sender of the call after the phase one.

T7: contains two processors with an artifact deployed on each one of them. The activity diagram contains two partitions interacting with each other using synchronous calls. The first partition contains a sequence of three activities starting with an InitialNode and sends two call requests to the second partition, to represent a PH1PH2 entry pattern, and the second partition contains one sequence of activities, and one activity graph, which contains control nodes of type fork and join. Reply messages are sent back from the sequence of activities and the activity graph of this partition to the senders of each call, one after phase one of the sequence, and the second after the last activity of the activity graph.

T8: contains two processors with an artifact deployed on each one of them. The activity diagram contains two partitions interacting with each other using both synchronous and asynchronous calls. The first partition contains a sequence of three activities starting with InitialNode and sends two call requests to the second partition, to represent a PH1PH2 entry pattern. The second partition contains two sequences of activities each starting with an AcceptEventAction to represent a $\mathrm{PH} 1 \mathrm{PH} 2$ entry pattern. Sending the reply message back from the first sequence of this partition to its sender of the call after its activity 
phase one ends that sequence, while there is no need to have a reply back from this partition to the sender of the asynchronous call after the first activity of the second sequence. The second sequence ends with an ActivityFinalNode.

The result of the testing is that all the tests have passed successfully.

For the validation of the transformation, we checked whether an important overall requirement (that the LQN model generated by the transformation can be correctly parsed by the existing LQN tools) is met. The result presented in the thesis show that the LQN solver can parse successfully the generated files and produce the expected results.

\subsection{Case study: E-commerce system}

The E-commerce case study (labeled here CS2) is taken from a book on Software Performance [Corte11], where the authors present the UML model of an e-commerce system, and then apply known techniques for building an LQN model. We decided to use a case study published by other people in order to reduce the author bias, where test cases built by the authors of a software product may select (willingly or unwillingly) test cases that work well with the software which is being tested.

The case-study contains three performance-critical use cases selected for performance analysis: Browse Catalogue, Browse Cart, and Place Order respectively. As there are N number of different customers in the system, $\mathrm{N}$ number of different instances of Customer Interface and Customer Process are running in the system every time.

Figure 6-1 presents the annotated UML deployment diagram of the system. The system has 9 local hosts. Each of the $\mathrm{N}$ users is deployed on its own User Device host and runs Customer Interface artifact on their own processor. Proc2, Proc4, and Proc5 are assigned to Customer Server, Cart Server and Catalog Server respectively. Proc3 is dedicated to 
the Delivery Order Process and Order Server, while all the $\mathrm{N}$ instances of Customer Process are deployed on Proc1.

Figures 6-2, 6-3, and 6-4 show the annotated UML activity diagrams for the three use cases mentioned above respectively. It is assumed that the system workload for each scenario is closed. Each step in the activity diagram is annotated by the $<<$ PaStep $>>$ stereotype, which means that the mean service demand required by the step from its processor (hostDemand) is given. As we can see in Figure 6-5, the LQN model generated by the LQNS toolset command lqn2emf illustrates that the transformation produced the expected result. We also checked that the XML format of the generated LQN model is correct, but did not include it in the thesis because of its length.
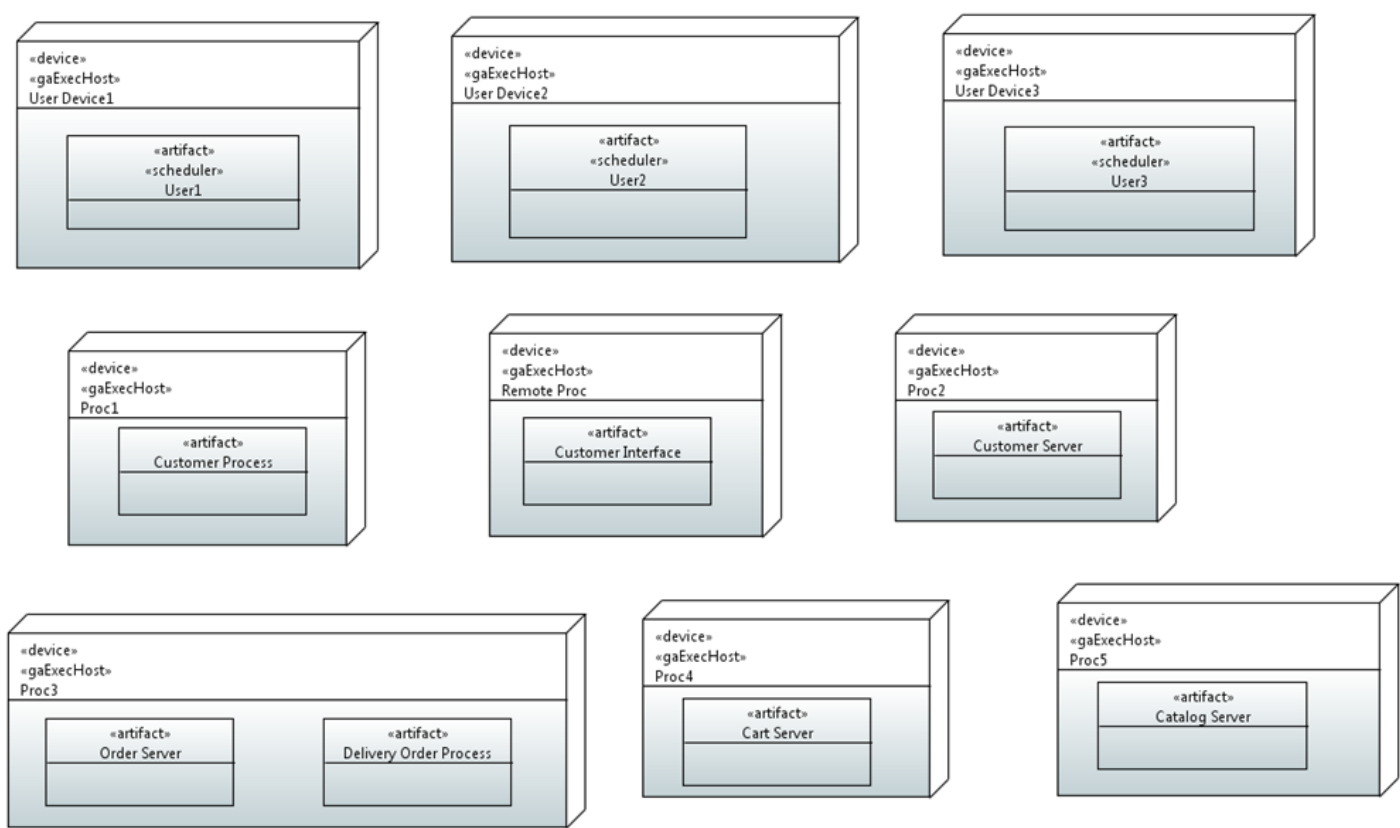

Figure 6-1. Deployment Diagram of E-commerce System 


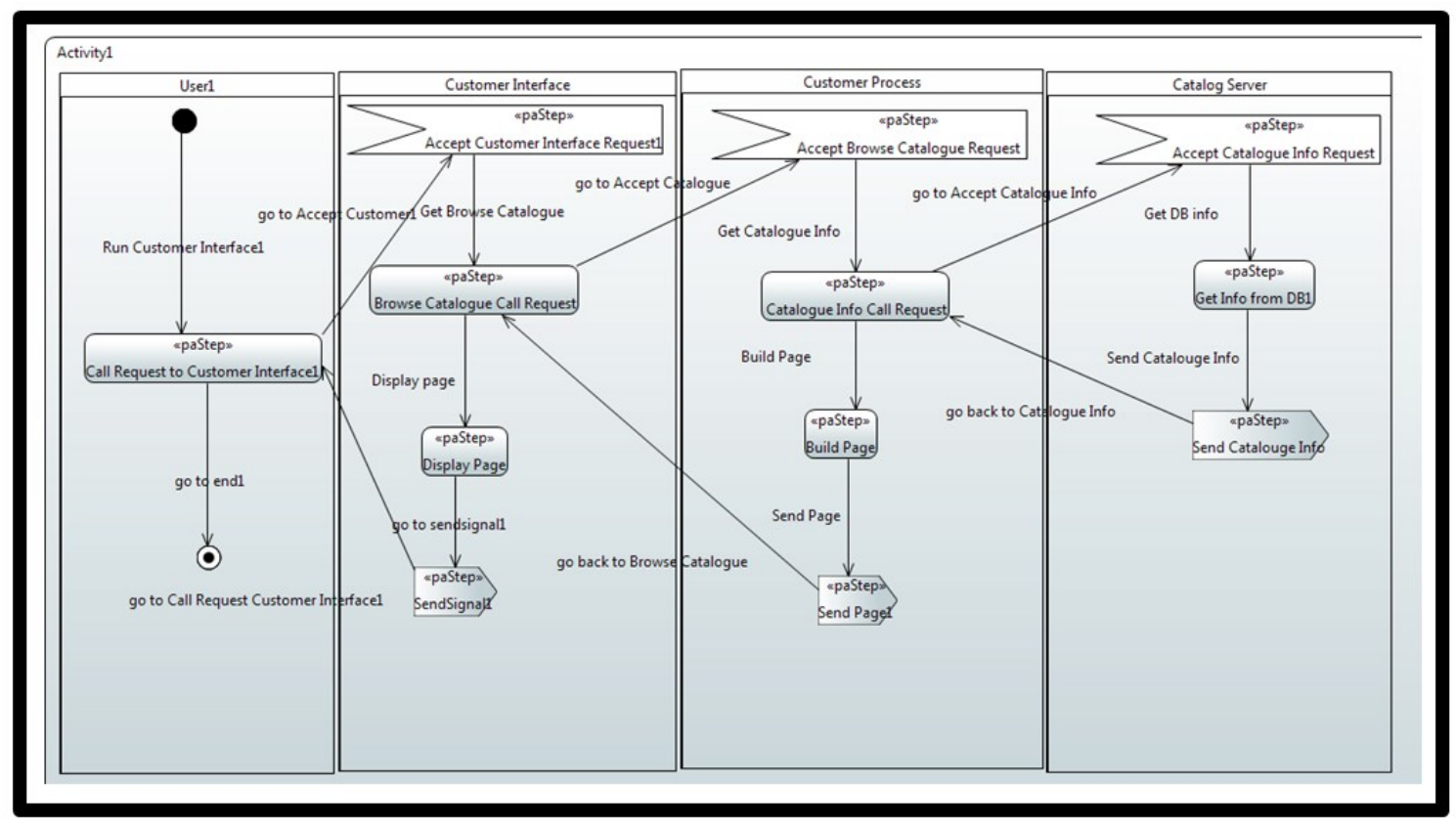

Figure 6-2. Activity Diagram of BrowseCatalog Scenario

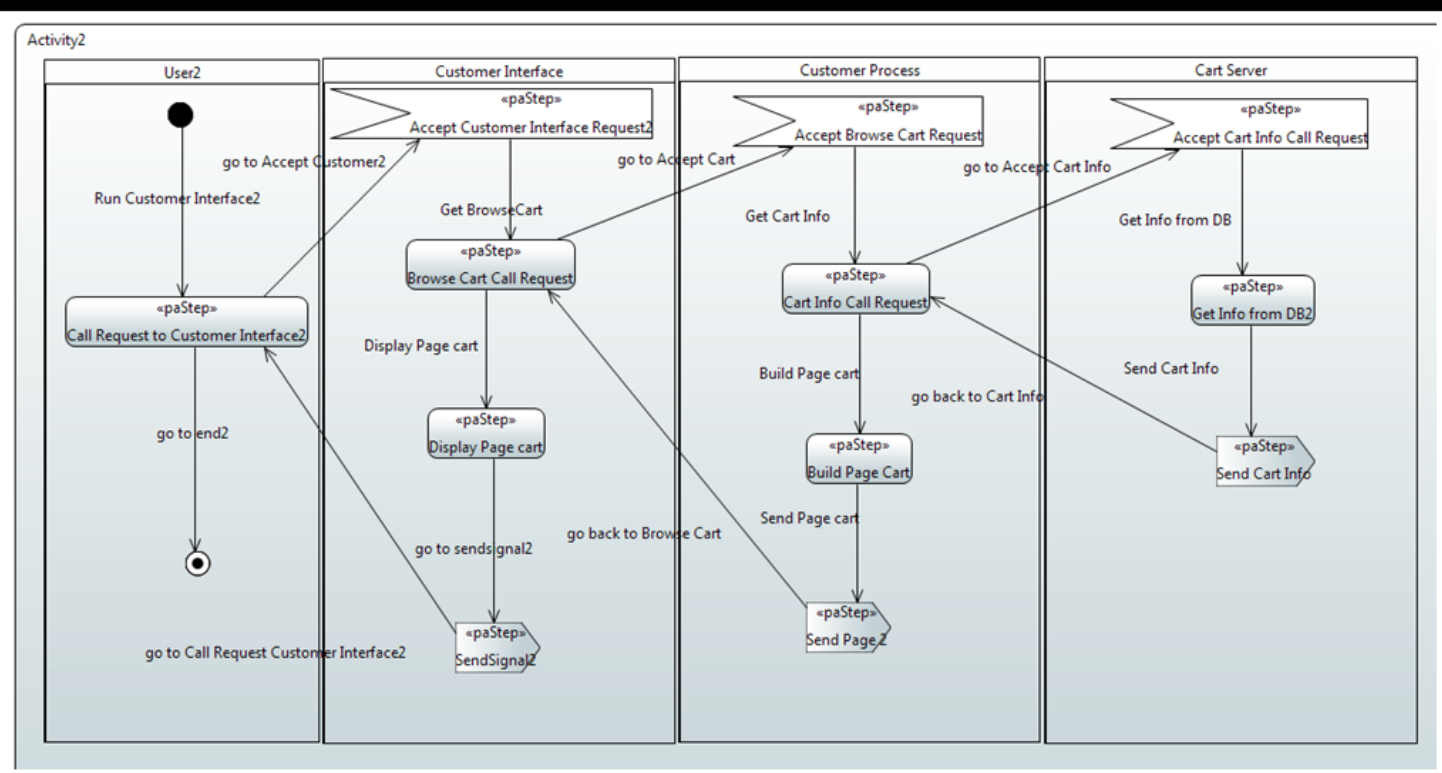

Figure 6-3. Activity Diagram of BrowseCart Scenario 


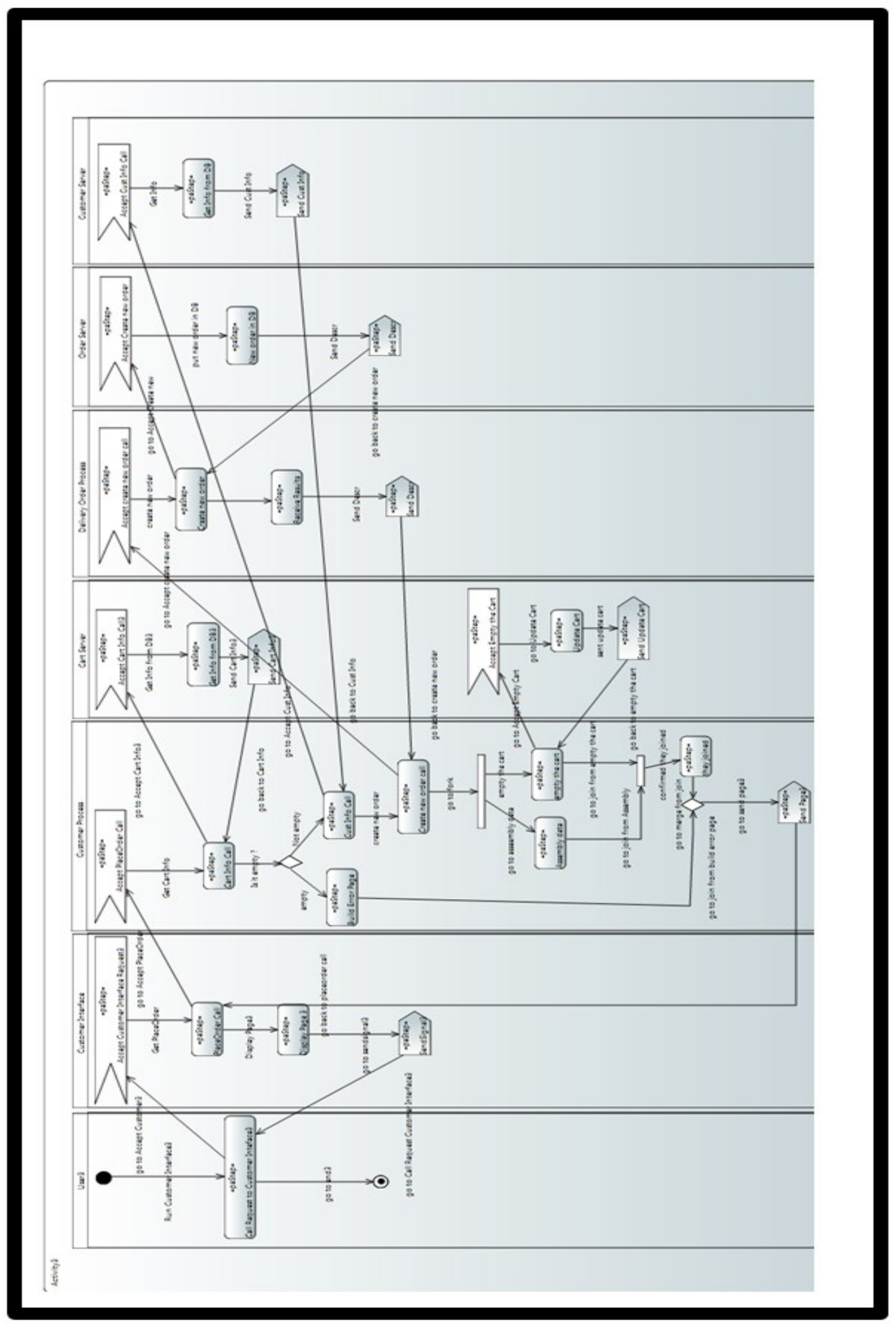

Figure 6-4. Activity Diagram of PlaceOrder Scenario 


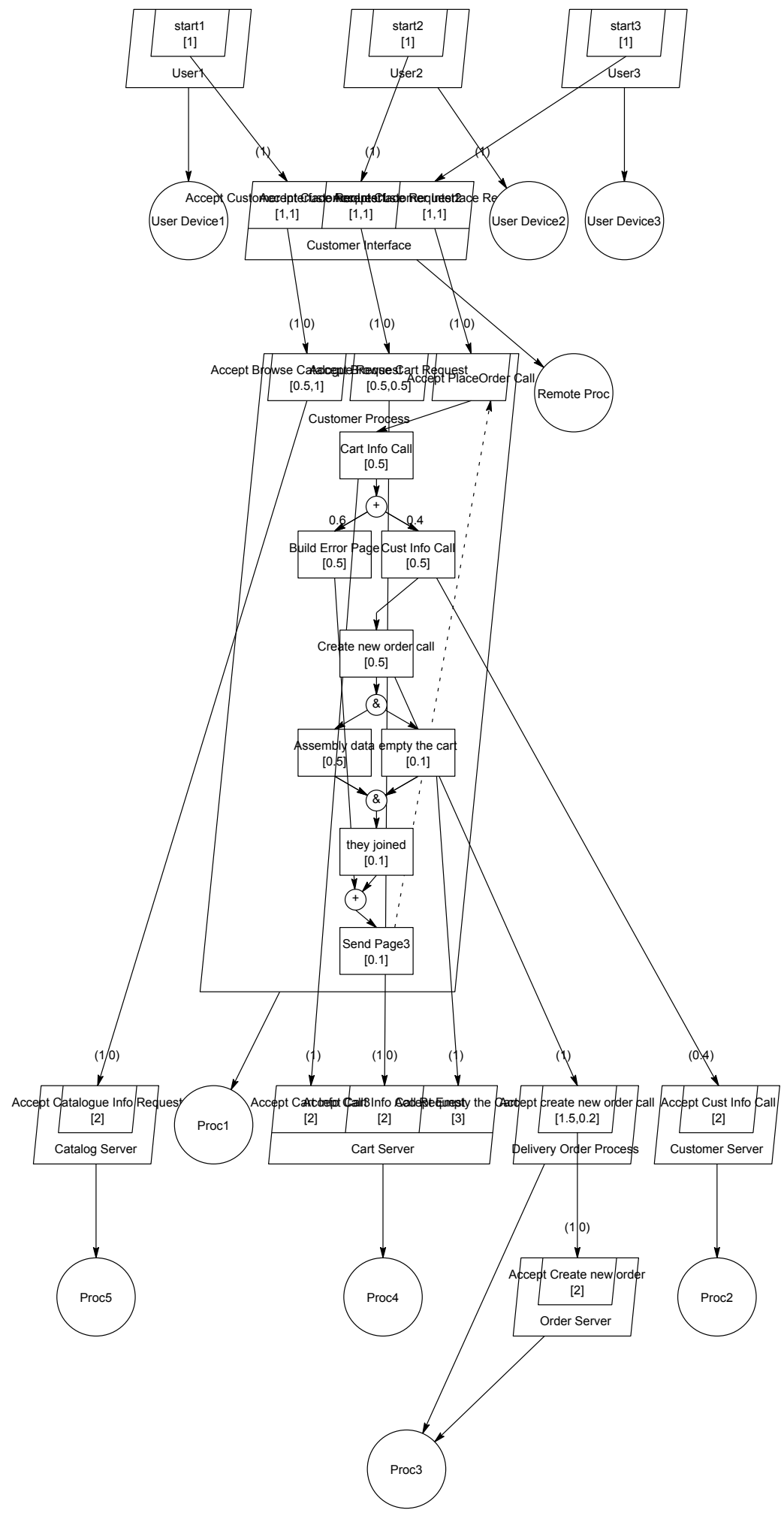

Figure 6-5. LQN Model of E-commerce System 


\section{Chapter: Conclusions}

\subsection{Completed work}

The high-level goal of this thesis is to contribute to the seamless integration of software performance engineering in the early stages of the model-driven software development process. This was achieved by meeting the concrete objectives of the thesis.

The first objective was to develop a model transformation process that takes as input a UML software model with MARTE performance annotations, and generates the corresponding Layered Queueing Network (LQN) performance model in a format understood by the existing LQN tools [LQN15]. The advantage of the one-step transformation from the software to the performance model is that direct cross-model trace links between source and target model can be defined, which allow us to import performance results obtained from the target model back to the source model. Also, incremental propagation of changes from the source to the target model, and co-evolution of the software and analysis model are supported by cross-model traceability.

The thesis developed and verified a multi-step process (see Figure 4-1), which meets this objective. The LQN models created by this process are read, parsed and solved by the LQN solvers. It is also possible to obtain the graphical notation of the LQN models by feeding the generated XML format to the appropriate translation commands. A lesson learned during the development of the main transformation in Step 3 (Figure 4-1) is that the LQN metamodel described in the Emfatic language had some differences from the original LQN metamodel based on the XML schema. This led to the introduction of the post-transformation processing step, which was not planned from the beginning. The pretransformation processing step was introduced to simplify the work of the human user. 
We realized that some of the derived attributes of the UML model are not set by the Papyrus tool. Instead of expecting the user to do the mechanical work of assigning values to these attributes, we decided that automating the work is a better solution. In fact, it is possible to extend this step with other verifications.

Another objective of the thesis was to experiment with the new family of languages, Epsilon, specialized in model transformations, refinement and management. After a quite steep learning slope, it became apparent that the Epsilon languages are powerful and help expressing the transformations rules that realize the mapping between source and target elements established by the designer. The OCL-like operations are at a good level of abstraction and help in writing complex conditions. A disadvantage of Epsilon is that the engine does a lot of background work and sometimes it is hard to debug the transformation code. As we found out, a lot of print-out statement at the beginning and ending of each rule are quite helpful. In many cases the documentation did not contain the information we were looking for; in such cases, we got good feedback from the online forums.

\subsection{Limitations}

The transformation implementation has some limitations, as follows:

- The transformation is rather sensitive to the input model assumptions listed in Chapter 3. If the input model does not follow the assumptions, the transformation stops running.

- There are two LQN features that are not implemented and need to be completed in future work: forwarding calls and loops. 
- Papyrus does not implement all the metaclasses defined in the UML metamodel yet, so we have to find some other solutions. For instance, AcceptCallAction and ReplyAction are not implemented, so we had to use other type of Actions to represent the receipt of a call and the reply. Also, in the case of deployment diagrams, the links between deployment nodes are not implemented, so one cannot model communication networks.

- The set of test cases could be extended with other models created by other authors. This would reduce the potential bias of the authors when creating new test cases.

- More checking of the input model could be done in Step 2. For instance, check whether the performance annotations are complete. If some values are missing, provide some pre-defined default values and also alert the user.

\subsection{Future work}

It is expected that the Epsilon transformation developed in the thesis can be used by future students in their research in the following directions:

- Extend the transformation to include more activity diagram features in the transformation (such as composite activity blocks).

- Extend the transformation to include scenarios defined by interaction diagrams.

- Import performance results from the LQNS solver output to the UML+MARTE model.

- Develop cross-model traceability to be used for the incremental propagation of changes from the software model to the performance model. The need for such tracelinks has been already identified in [Mani13. Mani15]. 
- Use cross-model trace links to support the co-evolution of the software and analysis model when the software model changes due to different reasons (correcting bugs, introducing new features etc.)

- Develop more pre-transformation verifications of the input model, to check whether the model is correct and complete and the transformation will run without problems. 


\section{Appendices}

\section{Appendix A LQN Metamodel in Emfatic language}

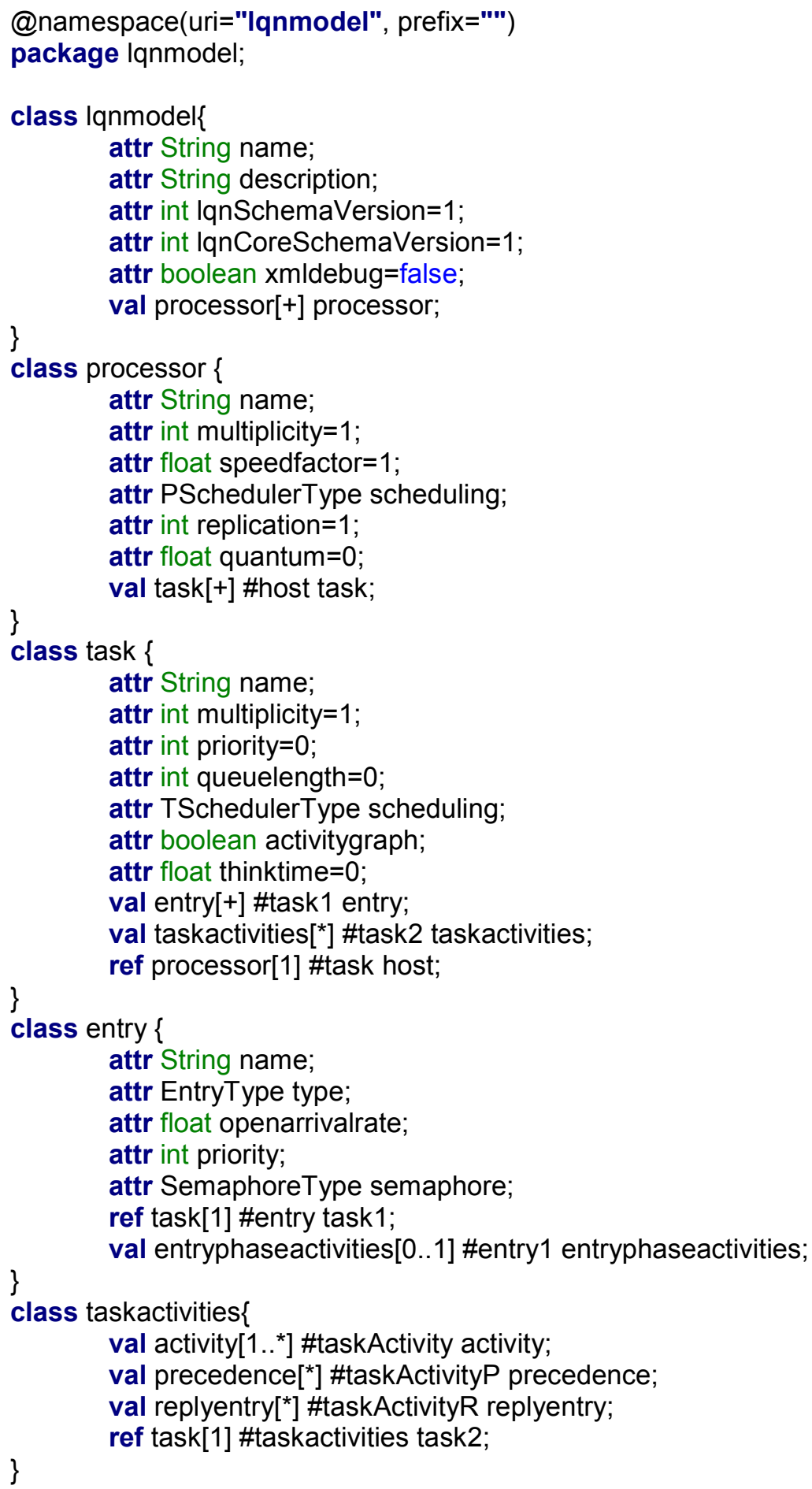




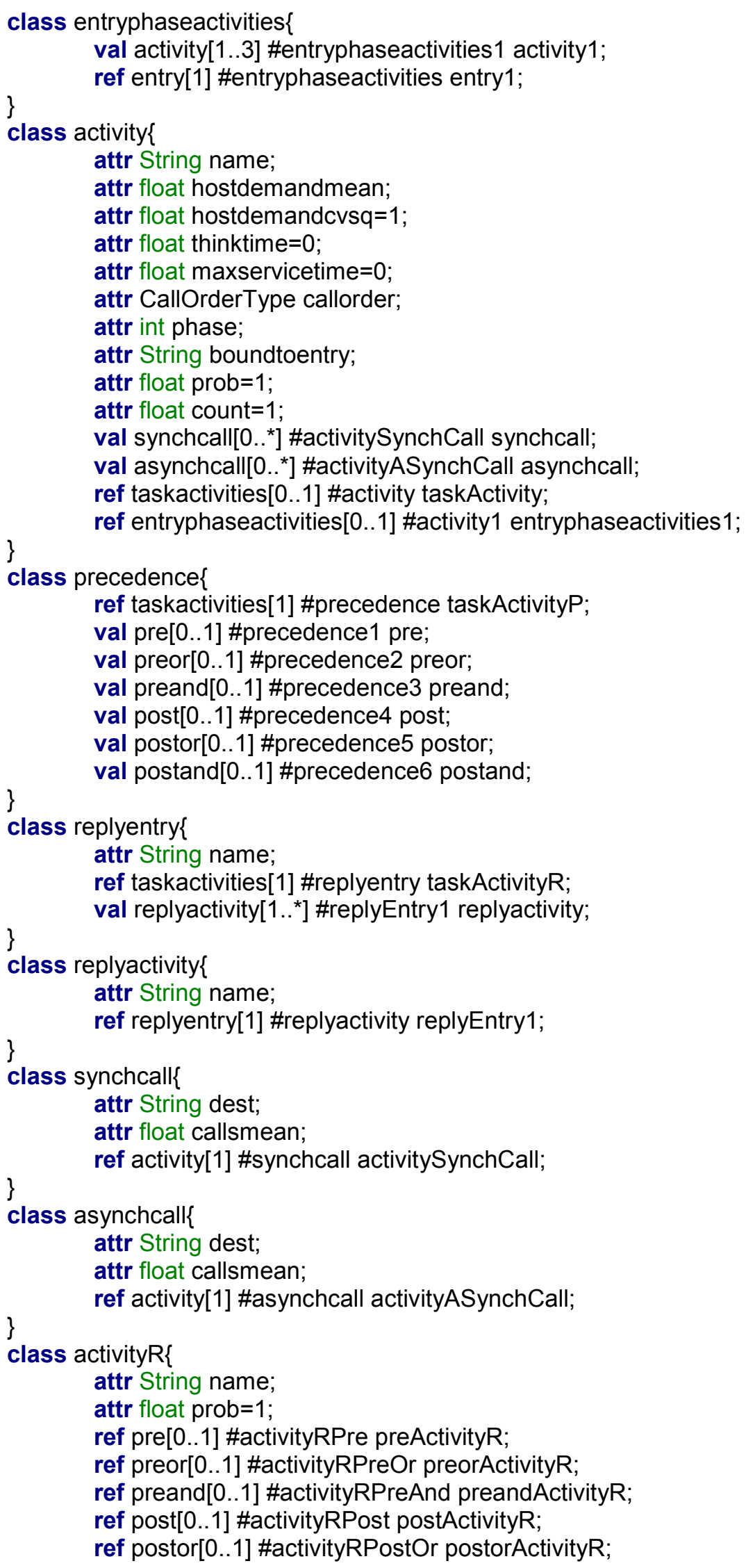




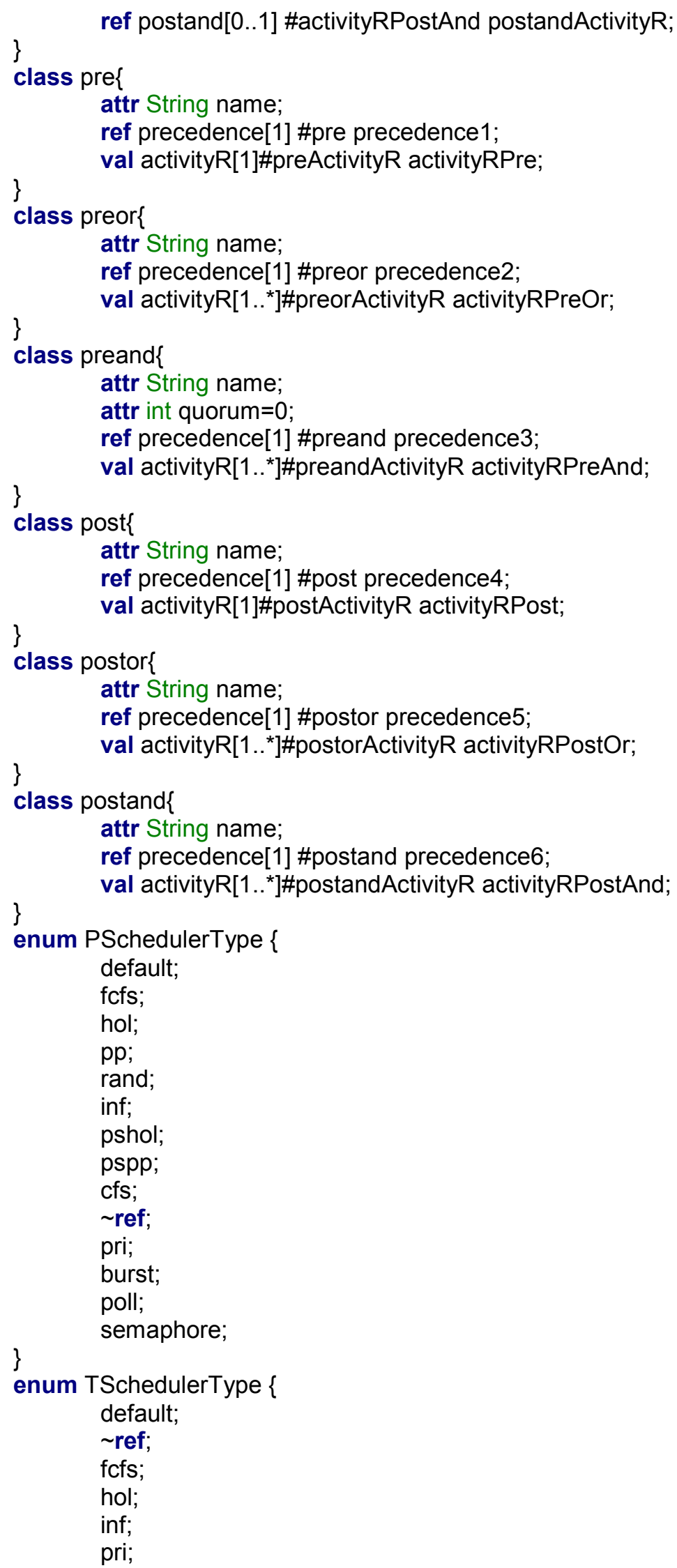




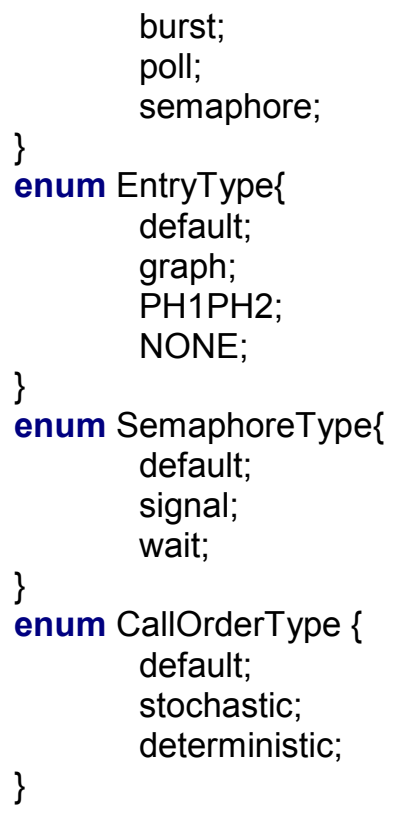




\section{Appendix B LQN model generated from the UML model from Figures 3-1 and 3-2}

\section{B.1. LQN model file generated by the Epsilon/ETL transformation}

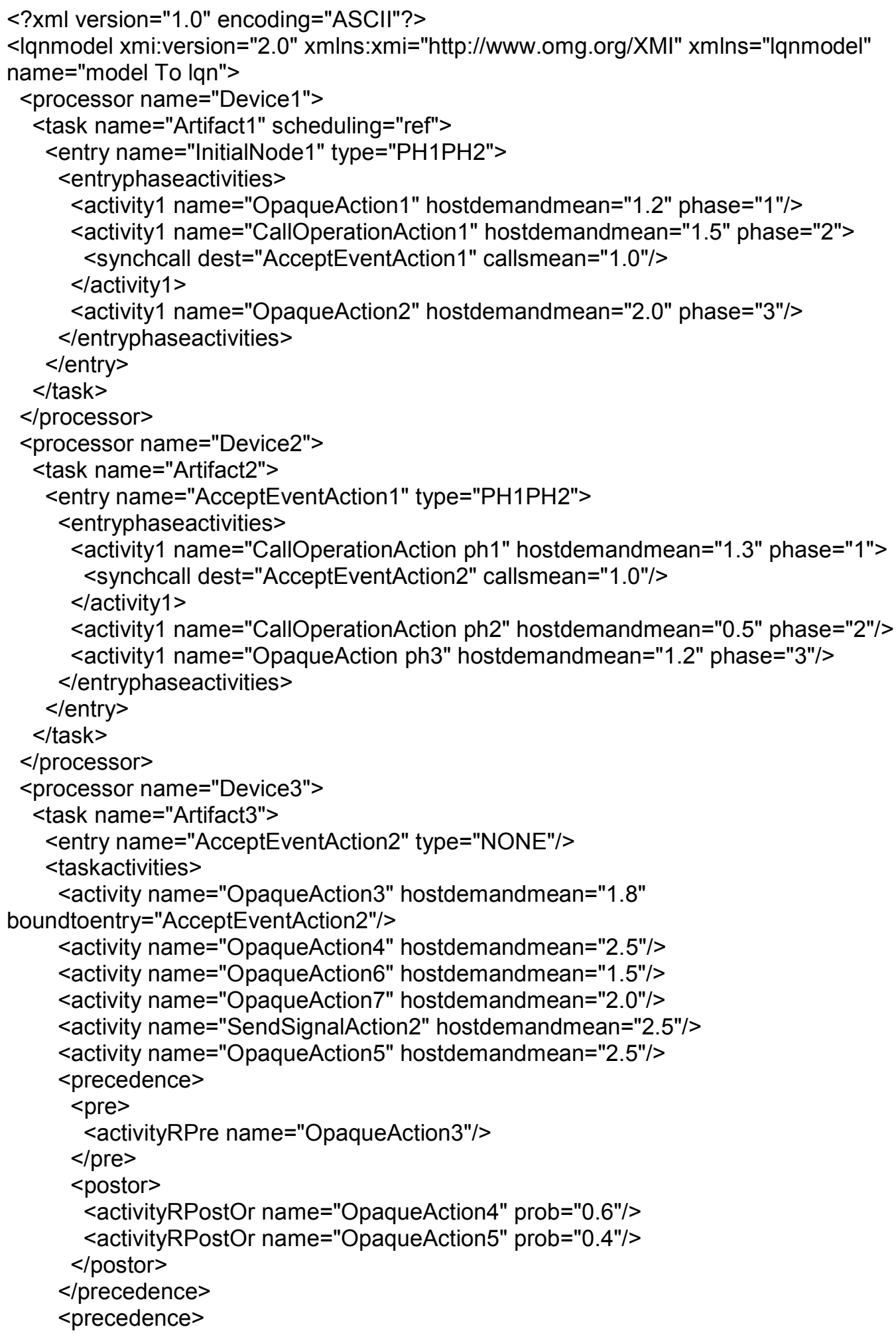




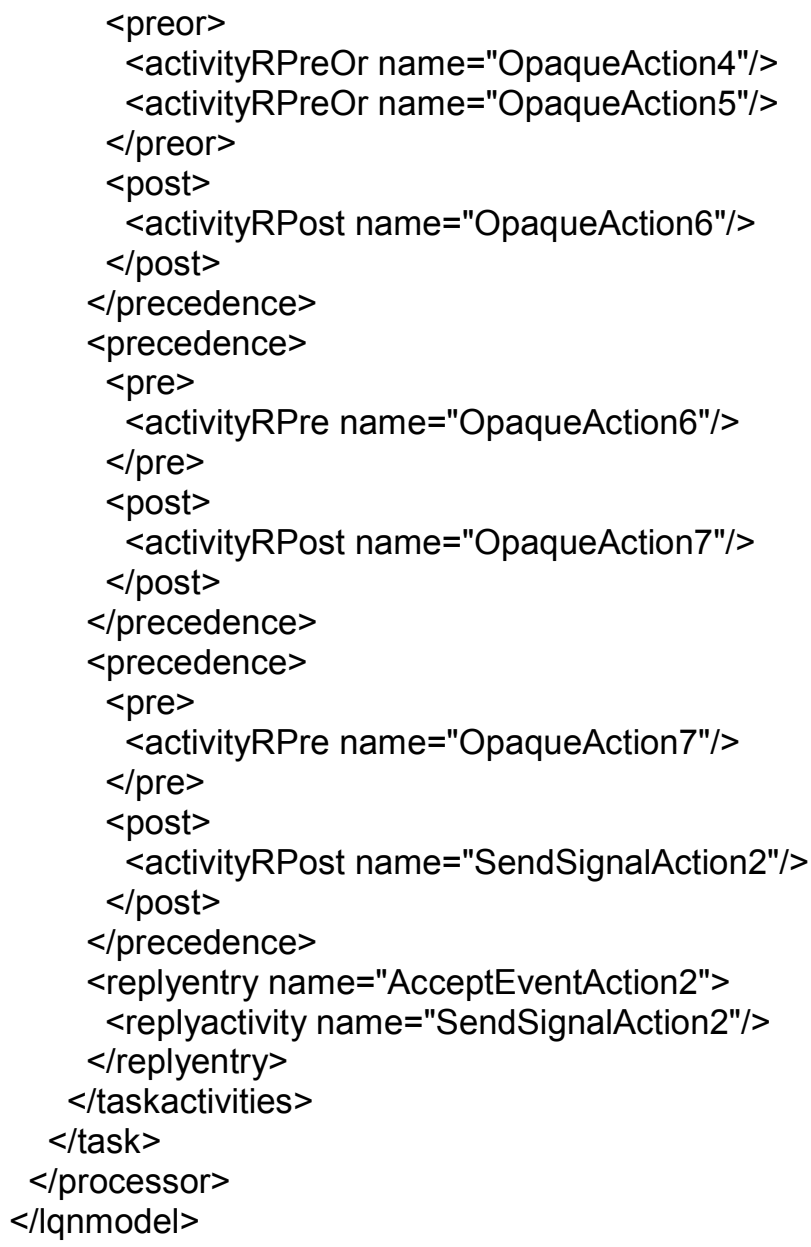

\section{B.2. LQN model file after post-transformation processing}

This format is accepted by the solver and its graphical notation is given in Figure 3-17.

$<$ ?xml version="1.0" encoding="ASCII"?>

<lqn-model xmlns:xsi="http://www.w3.org/2001/XMLSchema-instance" xsi:noNamespaceSchemaLocation="file:///M:/LQNSolvers/lqn.xsd" name="model To Iqn"> $<$ solver-params comment="Activity template" conv_val="1e-06" it_limit="50" 


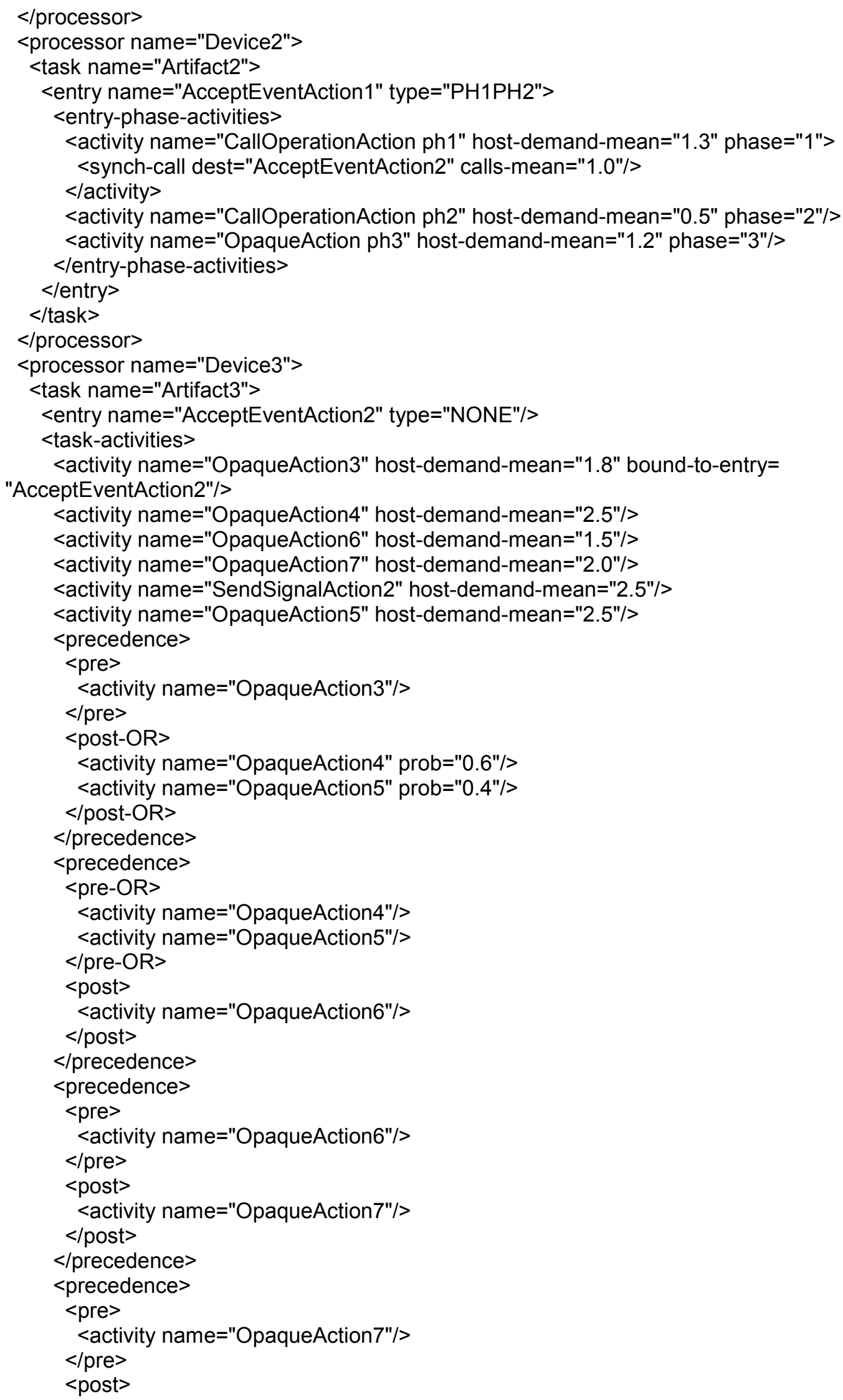




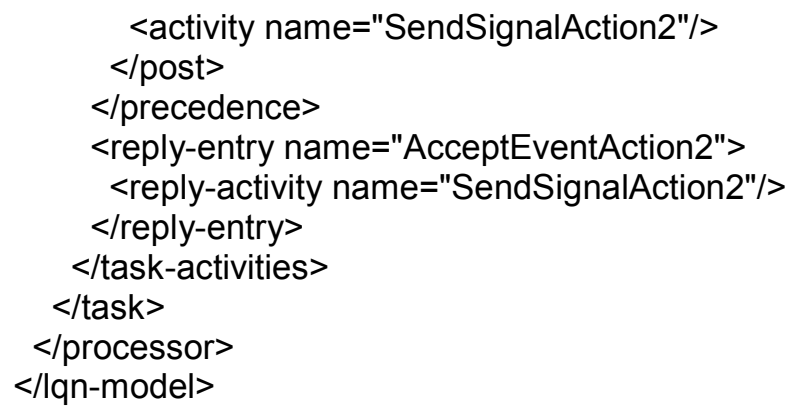

\section{B.3. LQN model output produced by the solver}

$<$ ?xml version="1.0"?>

$<!--$ Iqns -->

$<$ lqn-model name="LQNSolvers/MyExample/lqnFig3" description="lqns 5.8 solution for model from: LQNSolvers/MyExample/lqnFig3.Iqnx." xmlns:xsi="http://www.w3.org/2001/XMLSchemainstance" xsi:noNamespaceSchemaLocation="/usr/local/share/lqns/lqn.xsd">

<solver-params comment="Activity template" conv_val="1e-06" it_limit="50" underrelax_coeff="0.9" print int="5">

$<$ result-general valid="YES" conv-val="0" iterations="2" platform-info="delta.sce.carleton.ca Linux 2.6.32-504.12.2.el6.x86_64" user-cpu-time="00:00:00.00" system-cpu-time="00:00:00.00" elapsed-time="00:00:00.00">

$<$ mva-info submodels="3" core="6" step="33" step-squared="183" wait="488" wait-

squared="42262" faults="0"/>

$<$ result-general>

$<$ /solver-params $>$

$<$ processor name="Device1" scheduling="fcfs">

$<$ result-processor utilization="0.278404"/>

$<$ task name="Artifact1" scheduling="ref">

<result-task throughput="0.0592349" utilization="1" phase1-utilization="0.0710818" phase2-

utilization="0.810448" phase3-utilization="0.11847" proc-utilization="0.278404"/>

<entry name="InitialNode1" type="PH1PH2">

$<$ result-entry utilization="1" throughput="0.0592349" squared-coeff-variation="2.08777"

proc-utilization="0.278404" throughput-bound="0.0613497"/>

<entry-phase-activities>

<activity name="OpaqueAction1" phase="1" host-demand-mean="1.2">

$<$ result-activity proc-waiting="0" service-time="1.2" utilization="0.0710818" service-

time-variance="1.44"/>

$</ a c t i v i t y>$

<activity name="CallOperationAction 1 " phase="2" host-demand-mean="1.5">

$<$ result-activity proc-waiting="0" service-time="13.6819" utilization="0.810448"

service-time-variance="589.574"/>

<synch-call dest="AcceptEventAction1" calls-mean="1">

$<$ result-call waiting="0.581948"/>

$<$ /synch-call $>$

$</$ activity>

<activity name="OpaqueAction2" phase="3" host-demand-mean="2">

$<$ result-activity proc-waiting="0" service-time="2" utilization="0.11847" service-timevariance $=" 4 " />$

$</$ activity>

$<$ entry-phase-activities $>$

$</$ entry $>$

$</$ task $>$

$</$ processor $>$ 
<processor name="Device2" scheduling="fcfs">

$<$ result-processor utilization="0.177705"/>

$<$ task name="Artifact2" scheduling="fcfs">

<result-task throughput="0.0592349" utilization="0.787824" phase1-utilization="0.687124"

phase2-utilization="0.0296174" phase3-utilization="0.0710818" proc-utilization="0.177705"/>

<entry name="AcceptEventAction1" type="PH1PH2">

$<$ result-entry utilization="0.787824" throughput="0.0592349" squared-coeff-

variation="1.44344" proc-utilization="0.177705" throughput-bound="0.075188"/>

<entry-phase-activities>

<activity name="CallOperationAction ph1" phase="1" host-demand-mean="1.3">

$<$ result-activity proc-waiting="0" service-time="11.6" utilization="0.687124" service-

time-variance="253.64"/>

<synch-call dest="AcceptEventAction2" calls-mean="1">

$<$ result-call waiting="0"/>

$<$ /synch-call $>$

$<$ activity>

<activity name="CallOperationAction ph2" phase="2" host-demand-mean="0.5"> $<$ result-activity proc-waiting="0" service-time="0.5" utilization="0.0296174" service-

time-variance="0.25"/>

$</$ activity>

<activity name="OpaqueAction ph3" phase="3" host-demand-mean="1.2">

$<$ result-activity proc-waiting="0" service-time="1.2" utilization="0.0710818" service-

time-variance $=" 1.44 " />$

$<$ activity>

$</$ entry-phase-activities $>$

$<$ entry $>$

$<$ task $>$

$<$ processor $>$

<processor name="Device3" scheduling="fcfs">

$<$ result-processor utilization="0.610119"/>

$<$ task name="Artifact3" scheduling="fcfs">

$<$ result-task throughput="0.0592349" utilization="0.610119" phase1-utilization="0.610119"

proc-utilization="0.610119"/>

<entry name="AcceptEventAction2" type="NONE"> <result-entry utilization="0.610119" throughput="0.0592349" squared-coeff-

variation $=" 0.122443$ " proc-utilization $=" 0.610119$ " throughput-bound $=" 0.0970874 "$ phase 1 -service-

time="10.3" phase1-service-time-variance="12.99"/>

$<$ entry>

$<$ task-activities>

<activity name="OpaqueAction3" bound-to-entry="AcceptEventAction2" host-demand-

mean="1.8">

$<$ result-activity proc-waiting="0" service-time="1.8" utilization="0.106623" service-time-

variance $=" 3.24$ " throughput="0.0592349" proc-utilization="0.106623"/>

$<$ activity>

$<$ activity name="OpaqueAction4" host-demand-mean="2.5">

$<$ result-activity proc-waiting="0" service-time $=" 2.5$ " utilization="0.0888523" service-time-

variance="6.25" throughput="0.0355409" proc-utilization="0.0888523"/>

$<$ /activity>

$<$ activity name="OpaqueAction5" host-demand-mean="2.5">

$<$ result-activity proc-waiting="0" service-time $=" 2.5$ " utilization="0.0592349" service-time-

variance $=" 6.25$ " throughput="0.0236939" proc-utilization="0.0592349"/>

$<$ /activity>

<activity name="OpaqueAction6" host-demand-mean="1.5">

$<$ result-activity proc-waiting="0" service-time $=" 1.5$ " utilization="0.0888523" service-time-

variance $=" 2.25$ " throughput="0.0592349" proc-utilization="0.0888523"/>

$<$ /activity> 
<activity name="OpaqueAction7" host-demand-mean="2">

$<$ result-activity proc-waiting="0" service-time="2" utilization="0.11847" service-time-

variance="4" throughput="0.0592349" proc-utilization="0.11847"/>

$<$ activity>

<activity name="SendSignalAction2" host-demand-mean="2.5">

$<$ result-activity proc-waiting $=" 0$ " service-time $=" 2.5$ " utilization $=" 0.148087$ " service-time-

variance="6.25" throughput="0.0592349" proc-utilization="0.148087"/>

$<$ /activity>

$<$ precedence $>$

$<$ pre $>$

$<$ activity name="OpaqueAction3"/>

$<$ pre $>$

$<$ post-OR>

$<$ activity name="OpaqueAction4" prob="0.6"/>

$<$ activity name="OpaqueAction5" prob="0.4"/>

$<$ post-OR>

$</$ precedence $>$

$<$ precedence $>$

$<$ pre-OR>

<activity name="OpaqueAction4"/>

$<$ activity name="OpaqueAction5"/>

$<$ /pre-OR $>$

$<$ post $>$

<activity name="OpaqueAction6"/>

$<$ post $>$

$</$ precedence $>$

$<$ precedence $>$

$<$ pre $>$

$<$ activity name="OpaqueAction6"/>

$<$ pre $>$

$<$ post $>$

$<$ activity name="OpaqueAction7"/>

$<$ post $>$

$</$ precedence $>$

<precedence>

$<$ pre $>$

$<$ activity name="OpaqueAction7"/>

$</$ pre $>$

$<$ post $>$

$<$ activity name="SendSignalAction2"/>

$<$ post $>$

$</$ precedence $>$

$<$ reply-entry name="AcceptEventAction2">

$<$ reply-activity name="SendSignalAction2"/>

$<$ /reply-entry $>$

$<$ /task-activities $>$

$<$ task >

$</$ processor $>$

$</$ lqn-model $>$ 


\section{References}

[Ajmo95] M. Ajmone Marsan, G. Balbo G. Conte, S. Donatelli, G. Franceschinis, Modelling with Generalized Stochastic Petri Nets, Wiley Series in Parallel Computing, John Wiley and Sons, 1995.

[Alha10] M.Alhaj, D.C. Petriu, "Approach for generating performance models from UML models of SOA systems", Proc. of CASCON 2010, Toronto, 2010.

[Alha13] M. Alhaj, D. C. Petriu, "Traceability links in model transformations between software and performance models", In: SDL 2013: Model-Driven Dependability Engineering (eds. F. Khendek, M. Toeroe, A. Gherbi, R. Reed), LNCS, Vol. 7916, Pages 203-221, Springer, June 2013.

[Alha14] M. Alhaj, "Automatic Derivation of performance Models in the Context of Model-Driven SOA", Ph.D. Thesis, Department of Systems and Computer Engineering, Carleton University, January 2014.

[Amer01] "Automated Transformation of UML Software Specifications into LQN Performance Models using Graph-Grammar Techniques”, Master Thesis, Systems and Computer Engineering, Carleton University, May 2001.

[ATL12] "ATL User Guide", wiki.eclipse.org/ATL/User_Guide, 2012, last accessed Jan. 2016.

[Bals04] S. Balsamo, A. Di Marco, P. Inverardi, and M. Simeoni, "Model-based performance prediction in software development: a survey", IEEE Transactions on Software Engineering, vol. 30, N.5, pp.295-310, 2004.

[Cave04] C. Cavenet, S. Gilmore, J. Hillston, L. Kloul, L. and P. Stevens, "Analysing UML 2.0 activity diagrams in the software performance 
engineering process," in Proc. 4th Int. Workshop on Software and Performance (WOSP 2004), pp. 74-83, Redwood City, CA, Jan 2004.

[Cort00] V. Cortelessa, R. Mirandola, "Deriving a Queueing Network based Performance Model from UML Diagrams", in Proc. of 2nd ACM Workshop on Software and Performance (WOSP'20004), pp.58-70, Ottawa, Canada, 2000.

[Cort11] V. Cortellessa, A. Di Marco, P. Inverardi, Model-based Software Performance Analysis, Springer, 2011.

[Danc15] A.Danciu, J. Kroß, A. Brunnert, F. Willnecker, C. Vögele, A. Kapadia, and Helmut Krcmar, "Landscaping Performance Research at the ICPE and its Predecessors: A Systematic Literature Review", In Proceedings of the 6th ACM/SPEC International Conference on Performance Engineering (ICPE '15). ACM, New York, NY, USA, 91-96, 2015.

[EMF15] Eclipse Foundation, "Eclipse Modeling Framework (EMF)", www.eclipse.org/modeling/emf/, last visited Jan 2, 2016.

[Emfa14] Eclipse Foundation, "Emfatic Language Reference", www.eclipse.org/epsilon/doc/articles/emfatic/, last accessed Jan. 2016.

[Fran95] G. Franks, A. Hubbard, S. Majumdar, J. Neilson, D. Petriu, J. Rolia, M.Woodside, "A toolset for performance engineering and software design of client-server systems", Performance Evaluation 24 (1), 117-136, 1995.

[Fran99] R.G. Franks "Performance Analysis of Distributed Server Systems", PhD Thesis, Department of Systems and Computer Engineering, Carleton University, Ottawa, Ontario, Canada, December 1999. 
[Hill96] J. Hillston, A Compositional Approach to Performance Modelling. Cambridge University Press, 1996.

[Liu08] H. Liu, "Transformation of UML 2.0 Models Extended with MARTE to Core Scenario Models", M.S.(ISS) Thesis, Dept. of Systems and Computer Engineering, Carleton University, June 2008.

[LQN15] R.G.Franks, P. Maly, C.M. Woodside, D. C. Petriu, A. Hubbard, M.Mroz, "Layered Queueing Network Solver and Simulator User Manual", Department of Systems and Computer Engineering, Carleton University, 2015.

[Fran09] Greg Franks, Tariq Al-Omari, Murray Woodside, Olivia Das, and Salem Derisavi, "Enhanced modeling and solution of layered queueing networks", IEEE Transactions on Software Engineering, 35(2):148-161, 2009.

[Gu05] P.G. Gu, D.C. Petriu, "From UML to LQN by XML algebra-based model transformations", Proc. of the 5th ACM Workshop on Software and Performance (WOSP'05), pp. 99-110, Palma, Spain, July 11-14, 2005.

[Kol15] D. Kolovos, L. Rose, A. García-Domínguez, R. Paige, The Epsilon Book, http://www.eclipse.org/epsilon/doc/book/, last updated July 2015.

[Lope04] J.P. Lopez-Grao, J. Merseguer, J. Campos, "From UML Activity Diagrams To Stochastic Petri Nets: Application To Software Performance Engineering," in 4th Int. Workshop on Software and Performance (WOSP 2004), Redwood City, CA, (2004), 25-36.

[Lazo84] Lazowska E., Zahorjan J., Scott Graham G., Sevcik K.S., Quantitative 
System Performance: Computer System Analysis Using Queueing Network Models, Prentice Hall, 1984.

[MART11] Object Management Group, "UML Profile for MARTE: Modeling and Analysis of Real-Time Embedded Systems, Version 1.1, OMG doc. formal/2011-06-02, 2011.

[Mani13] N. Mani, D. C. Petriu, C.M. Woodside, "Propagation of Incremental Changes to Performance Model due to SOA Design Pattern Application“, Proc. of the ACM/SPEC International Conference on Software Engineering ICPE’2013, pp. 89-100, Prague, Czech Republic, April 2124, 2013.

[Mani15] N. Mani, "Studying the Performance Impact of SOA Design Patterns via Coupled Model Transformations", Ph.D. Thesis, Dept. of Systems and Computer Engineering, Carleton University, May 2015.

[MOF11] Object Management Group, "Meta Object Facility (MOF) 2.0 Query/View/Transformation Specification Version 1.1", OMG Document: formal/2011-01-01, January 2011.

[Papy15] Eclipse Foundation, "Papyrus Modeling Environment", eclipse.org/papyrus, last accessed January 2016.

[Petr02] D.C. Petriu, H. Shen, "Applying the UML Performance Profile: Graph Grammar based derivation of LQN models from UML specifications", in Computer Performance Evaluation - Modelling Techniques and Tools, (T.Fields, P. Harrison, J. Bradley, U. Harder, Eds.) Springer LNCS Vol. 2324, pp.159-177, 2002. 
[Petr07] D.B. Petriu, M. Woodside, "An intermediate metamodel with scenarios and resources for generating performance models from UML designs", Software and Systems Modeling, Volume 6, Nb. 2, pp.163-184, 2007.

[Petr14] D.B. Petriu, "CSM2LQN - Transformations for the Generation of Performance Models from Software Designs”, Ph.D. Thesis, Department of Systems and Computer Engineering, Carleton University, 2014.

[Petr15] D.C. Petriu, "Challenges in Integrating the Analysis of Multiple NonFunctional Properties in Model-Driven Software Engineering", in Proceedings of WOSP-C'15, Austin, TX, USA, January 2015.

[Plat91] Plateau B., Atif K., "Stochastic Automata Network of Modeling Parallel Systems", IEEE Transactions on Software Engineering, Vol.17, No.10, p.1093-1108, 1991.

[Pola16] Eclipse Foundation, PolarSys, www.polarsys.org, last accesses Jan. 2016

[Roli95] J. A. Rolia, K. A. Sevcik, "The method of layers", IEEE Transactions on Software Engineering, 21(8):689-700, August 1995

[QVT11] Object Management Group, "Meta Object Facility (MOF) 2.0 Query/View/Transformation Specification Version 1.1", OMG Document: formal/2011-01-01, January 2011.

[Seli13] B. Selic, S. Gérard, Modeling and Analysis of Real-Time and Embedded Systems with UML and MARTE: Developing Cyber-Physical Systems Morgan Kauffmann Publisher, 2013.

[Smit90] C.U. Smith, Performance Engineering of Software Systems, Reading Mass., Addison Wesley, 1990. 
[Smit01] C.U. Smith, L. Williams. Performance Solutions: A practical guide to creating responsive, scalable software, Addison-Wesley, 2001.

[SPT05] Object Management Group, "UML Profile for Schedulability, Performance, and Time Specification", version 1.1, OMG document: formal/05-01-02, January 2005.

[UML15] Object Management Group, "OMG Unified Modeling Language" Version 2.5, OMG document formal-15-03-01, 2015.

[Wood95] C. M.Woodside, J.E. Neilson, D.C. Petriu, S. Majumdar. "The stochastic rendezvous network model for performance of synchronous client-serverlike distributed software", IEEE Trans. on Computers, 44(8):20-34, 1995.

[Wood05] C.M. Woodside, D. C. Petriu, D. B. Petriu, Hui Shen, T. Israr, J. Merseguer, "Performance by Unified Model Analysis (PUMA)", Proc. of the 5th ACM Workshop on Software and Performance, pp.1-12, Palma, Spain, July 2005.

[Wood14] C.M. Woodside, D.C. Petriu, J. Merseguer, D.B. Petriu, M. Alhaj, "Transformation challenges: from software models to performance models", Software and Systems Modeling, Vol. 13, No. 4 (2014), Page $1529-1552$.

[XMI15] Object Management Group, "XML Metadata Interchange (XMI)" Version 2.5.1, OMG document formal/ 2015-06-07, 2015.

[XML08] W3C, “Extensible Markup Language (XML) 1.0”, 2008, www.w3.org/TR/xml/, last accessed Jan 2016. 